\title{
Auslander's formula and correspondence for exact categories
}

\author{
Ruben Henrard \\ Hasselt University
}

\author{
Sondre Kvamme \\ Uppsala Universitet
}

\author{
Adam-Christiaan van Roosmalen \\ Hasselt University
}

December 1, 2020

\begin{abstract}
The Auslander correspondence is a fundamental result in Auslander-Reiten theory. In this paper we introduce the category $\bmod _{a d m}(\mathcal{E})$ of admissibly finitely presented functors and use it to give a version of Auslander correspondence for any exact category $\mathcal{E}$. An important ingredient in the proof is the localization theory of exact categories. We also investigate how properties of $\mathcal{E}$ are reflected in $\bmod _{a d m}(\mathcal{E})$, for example being (weakly) idempotent complete or having enough projectives or injectives. Furthermore, we describe $\bmod _{\text {adm }}(\mathcal{E})$ as a subcategory of $\bmod (\mathcal{E})$ when $\mathcal{E}$ is a resolving subcategory of an abelian category. This includes the category of Gorenstein projective modules and the category of maximal Cohen-Macaulay modules as special cases. Finally, we use $\bmod _{\text {adm }}(\mathcal{E})$ to give a bijection between exact structures on an idempotent complete additive category $\mathcal{C}$ and certain resolving subcategories of $\bmod (\mathcal{C})$.
\end{abstract}

\section{Contents}

1 Introduction

2 Localizations/Quotients of exact categories 5



2.2 Localizations via two-sided admissibly percolating subcategories . . . . . . . . . . . 6

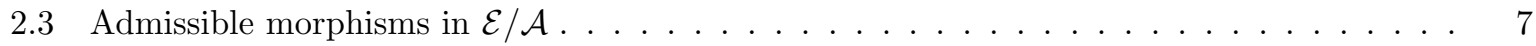

2.4 Torsion theory with two-sided admissibly percolating subcategories $\ldots \ldots \ldots \ldots \ldots$

3 Auslander's formula for exact categories $\quad 10$

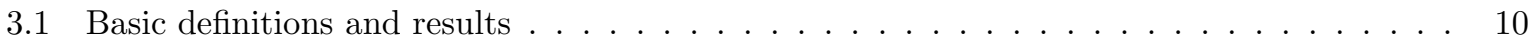

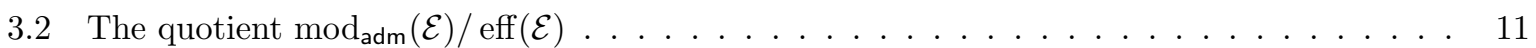

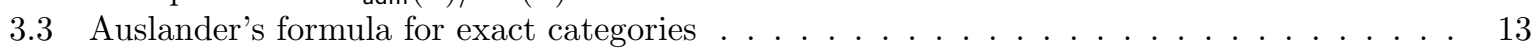

3.4 The torsion pair $(\operatorname{eff}(\mathcal{E}), \operatorname{cogen}(\mathcal{Q}))$ in $\bmod _{\mathrm{adm}}(\mathcal{E}) \ldots \ldots \ldots \ldots \ldots \ldots \ldots$

3.5 Admissibly presented functors and the category of sheaves . . . . . . . . . . . . . 17

4 Auslander correspondence for exact categories 19

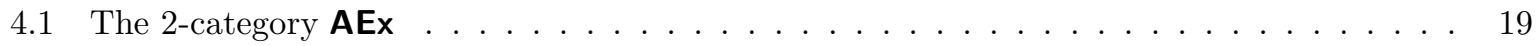

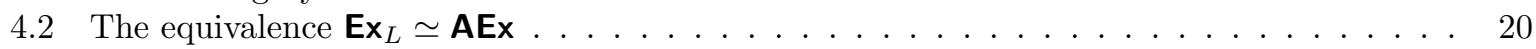

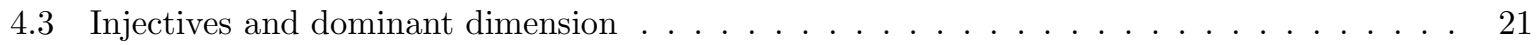



4.5 Gorenstein projectives and Cohen-Macaulay modules . . . . . . . . . . . . . . . 26

5 Characterizing exact structures via resolving subcategories $\quad 28$

5.1 Resolving subcategories . . . . . . . . . . . . . . . . . . . . . . . 28

5.2 The Auslander-Bridger transpose . . . . . . . . . . . . . . . . . . . . . . 28

5.3 Main theorem . . . . . . . . . . . . . . . . . . . . . . 30 


\section{Introduction}

To study representations of finite-dimensional algebras, Auslander advocated a functorial point of view, which he developed in $[6,7,10,11,12]$. This means that to study the category $\bmod (\Lambda)$ of finitedimensional modules over a finite-dimensional algebra $\Lambda$, one should study the category $\bmod (\bmod (\Lambda))$ of finitely presented functors $F: \bmod (\Lambda)^{\mathrm{op}} \rightarrow$ Ab to abelian groups, and translate the results back to $\bmod (\Lambda)$. For example, almost split sequences in $\bmod (\Lambda)$ naturally arise when taking minimal projective presentations of simple objects of projective dimension 2 in $\bmod (\bmod (\Lambda))$, and representation finiteness of $\Lambda$ can be detected by investigating when $\bmod (\bmod (\Lambda))$ is equivalent to $\bmod (\Gamma)$ for some finite-dimensional algebra $\Gamma$. In the latter case, $\Gamma$ inherits homological properties from the categorical properties of $\bmod (\bmod (\Lambda))$. More precisely, the class of algebras $\Gamma$ is equal to the class of Auslander algebras, i.e. the algebras satisfying

$$
\text { dom. } \operatorname{dim} \Gamma \geq 2 \geq \text { gl. } \operatorname{dim} \Gamma
$$

where $\operatorname{dom} . \operatorname{dim} \Gamma$ is the dominant dimension and $\operatorname{gl} \operatorname{dim} \Gamma$ is the global dimension of $\Gamma$. This recovers the famous Auslander correspondence.

On the other hand, in representation theory one often considers categories which are not abelian. If the category $\bmod (\Lambda)$ is too complicated, one might need to restrict to studying subcategories of $\bmod (\Lambda)$, for example the Gorenstein projective modules [16, 19, 28, 29, 59], or the modules of finite projective dimension [15, 41, 52, 55]. Furthermore, Cohen-Macaulay representations [7, 8, 23, 27, 46, 49, 51, 58, 65], their graded counterparts [13, 14], and their connection to McKay quivers [1,9] have also been actively studied from a representation theoretic point of view. In all these cases, the categories are exact in the sense of Quillen, but not necessarily abelian. Unfortunately, if $\mathcal{E}$ is an exact category, then $\bmod (\mathcal{E})$ does not have nice categorical properties in general, see [34,45]. This makes it harder to deduce results about $\mathcal{E}$ by studying $\bmod (\mathcal{E})$, as in Auslander's philosophy. In this paper we introduce a different candidate to study instead of $\bmod (\mathcal{E})$, which takes into account the exact structure of $\mathcal{E}$.

Definition 1.1 (Definition 3.1). Let $\mathcal{E}$ be an exact category. We define $\bmod _{\text {adm }}(\mathcal{E})$ to be the full subcategory of $\bmod (\mathcal{E})$ consisting of those functors $F$ that admit a projective representation

$$
\operatorname{Hom}_{\mathcal{E}}(-, X) \stackrel{\operatorname{Hom}_{\mathcal{E}}(-, f)}{\longrightarrow} \operatorname{Hom}_{\mathcal{E}}(-, Y) \rightarrow F \rightarrow 0
$$

where $f: X \rightarrow Y$ is an admissible morphism in $\mathcal{E}$.

We first characterize the categories of the form $\bmod _{a d m}(\mathcal{E})$, analogously to the characterization of Auslander algebras. This is done by introducing the notion of an Auslander exact category. In the following we write

$$
{ }^{\perp} \mathcal{P}:=\left\{E \in \mathcal{E} \mid \operatorname{Hom}_{\mathcal{E}}(E, \mathcal{P})=0\right\} \quad \text { and } \quad \operatorname{cogen}(\mathcal{P}):=\{E \in \mathcal{E} \mid \exists \text { inflation } E \longmapsto P \text { with } P \in \mathcal{P}\} .
$$

Definition 1.2 (Definition 4.1). Let $\mathcal{E}$ be an exact category with enough projectives $\mathcal{P}$. We say that $\mathcal{E}$ is an Auslander exact category if it satisfies the following:

(i) $\left({ }^{\perp} \mathcal{P}, \operatorname{cogen}(\mathcal{P})\right)$ is a torsion pair in $\mathcal{E}$;

(ii) If $E^{\prime} \in{ }^{\perp} \mathcal{P}$, then any morphism $f: E \rightarrow E^{\prime}$ is admissible with image in ${ }^{\perp} \mathcal{P}$;

(iii) $\operatorname{Ext}_{\mathcal{E}}^{1}\left({ }^{\perp} \mathcal{P}, \mathcal{P}\right)=0$

(iv) $\operatorname{gl} \operatorname{dim}(\mathcal{E}) \leq 2$.

Theorem 1.3 (Theorem 4.7). The following hold:

1. If $\mathcal{E}$ is an exact category, then $\bmod _{\mathrm{adm}}(\mathcal{E})$ is an Auslander exact category.

2. If $\mathcal{E}^{\prime}$ is an Auslander exact category, then there exists an exact equivalence $\mathcal{E}^{\prime} \cong \bmod _{\text {adm }}(\mathcal{E})$ where $\mathcal{E}$ is an exact category. Furthermore, $\mathcal{E}$ is uniquely determined up to exact equivalence. 
In fact, we show that the association $\mathcal{E} \mapsto \bmod _{\mathrm{adm}}(\mathcal{E})$ is an equivalence from the 2-category of exact categories and left exact functors to the 2-category of Auslander exact categories and exact functors preserving projective objects. It can also be interpreted as a left adjoint to an inclusion of 2-categories, see Corollary 3.12.

Note that if $\mathcal{Q}$ is the subcategory of projective objects in $\bmod _{\mathrm{adm}}(\mathcal{E})$, then we have the following identifications:

1. $F \in \mathcal{Q}$ if and only if $F$ is isomorphic to a representable functor.

2. $F \in{ }^{\perp} \mathcal{Q}$ if and only if $F$ is an effaceable functor, i.e. there exist a projective representation

$$
\operatorname{Hom}_{\mathcal{E}}(-, X) \stackrel{\operatorname{Hom}_{\mathcal{E}}(-, f)}{\longrightarrow} \operatorname{Hom}_{\mathcal{E}}(-, Y) \rightarrow F \rightarrow 0
$$

where $f: X \rightarrow Y$ is a deflation. These functors have already been studied by several authors under the name defects, see $[22,34,36]$.

3. $F \in \operatorname{cogen}(\mathcal{Q})$ if and only if there exist a projective representation

$$
\operatorname{Hom}_{\mathcal{E}}(-, X) \stackrel{\operatorname{Hom}_{\mathcal{E}}(-, f)}{\longrightarrow} \operatorname{Hom}_{\mathcal{E}}(-, Y) \rightarrow F \rightarrow 0
$$

where $f: X \rightarrow Y$ is an inflation.

It follows that the subcategory of effaceable functors is the torsion part of a torsion pair in $\bmod _{\text {adm }}(\mathcal{E})$. In fact, it is a two-sided admissibly percolating subcategory of $\bmod _{a d m}(\mathcal{E})$, which enables us to use the localisation theory developed in [26] and [43] to study it. In particular, we extend Auslander's formula to exact categories $[6,50]$.

Theorem 1.4 (Proposition 3.5 and Theorem 3.9). The following holds:

1. $\operatorname{eff}(\mathcal{E})$ is a two-sided admissibly percolating subcategory of $\bmod _{\mathrm{adm}}(\mathcal{E})$. In particular, the quotient $\bmod _{\mathrm{adm}}(\mathcal{E}) / \operatorname{eff}(\mathcal{E})$ exists (in the category of exact categories);

2. $\bmod _{\mathrm{adm}}(\mathcal{E}) / \operatorname{eff}(\mathcal{E})$ is equivalent to $\mathcal{E}$ as an exact category.

If $\mathcal{E}$ is idempotent complete, then we also show that $\bmod _{\mathrm{adm}}(\mathcal{E})$ is the smallest resolving subcategory containing $\operatorname{eff}(\mathcal{E})$.

If $\mathcal{E}$ has enough injectives, then we obtain a characterization of $\bmod _{\mathrm{adm}}(\mathcal{E})$ which is similar to module categories of Auslander algebras.

Theorem 1.5 (Theorem 4.21). Let $\mathcal{E}^{\prime}$ be an exact category with enough projectives $\mathcal{P}^{\prime}$. Then there exists an exact equivalence $\mathcal{E}^{\prime} \cong \bmod _{\mathrm{adm}}(\mathcal{E})$ where $\mathcal{E}$ is an exact category with enough injectives if and only if the following hold:

1. $\operatorname{dom} \cdot \operatorname{dim}\left(\mathcal{E}^{\prime}\right) \geq 2 \geq$ gl. $\operatorname{dim}\left(\mathcal{E}^{\prime}\right)$;

2. Any morphism $X \rightarrow E$ with $E \in{ }^{\perp} \mathcal{P}^{\prime}$ is admissible;

3. For any $E \in \mathcal{E}^{\prime}$ there exists an admissible left $\mathcal{P}^{\prime}$-approximation $E \rightarrow P$.

Furthermore, in this case $\mathcal{E}^{\prime}$ has enough injectives.

We also analyse the properties of $\bmod _{\mathrm{adm}}(\mathcal{E})$ when $\mathcal{E}$ has enough projectives, see Section 4.4.

One of our main examples of an exact category $\mathcal{E}$ is the category of Cohen-Macaulay modules, and it turns out that $\bmod _{\mathrm{adm}}(\mathcal{E})$ has a simple description in this case. Let $R$ be a commutative Cohen-Macaulay local ring and let $\Lambda$ be a Cohen-Macaulay-finite $R$-order, i.e. a noetherian $R$-algebra which is maximal Cohen-Macaulay as an $R$-module. Let $M$ be an additive generator of the subcategory $\operatorname{CM}(\Lambda)$ of maximal Cohen-Macaulay modules containing $\Lambda$ as a summand. Let $\Gamma=\operatorname{End}_{\Lambda}(M)$ and let $e$ be the idempotent corresponding to the direct summand $\Lambda$. Then we have

$$
\bmod _{\mathrm{adm}}(\Gamma)=\{M \in \bmod (\Gamma) \mid M e \in \mathrm{CM}(\Lambda)\} .
$$


Similar descriptions hold for any resolving subcategory of a module category.

The study of exact structures on a fixed idempotent complete additive category $\mathcal{C}$ has recently attracted a lot of interest $[17,20,21,34,36,61,62,63]$. In particular, they form a lattice $[21,61]$ and the association $\mathcal{E} \mapsto \operatorname{eff}(\mathcal{E})$ gives a bijection to certain Serre subcategories of $\bmod (\mathcal{E})[22,34,36]$. We obtain a similar characterization by replacing $\operatorname{eff}(\mathcal{E})$ with $\bmod _{\text {adm }}(\mathcal{E})$ and Serre subcategories by resolving subcategories. In the following, $\mathcal{P}^{2}(\mathcal{C})$ denotes the subcategory of $\bmod (\mathcal{C})$ consisting of functors $F$ admitting a projective resolution

$$
0 \rightarrow \operatorname{Hom}_{\mathcal{E}}(-, X) \rightarrow \operatorname{Hom}_{\mathcal{E}}(-, Y) \rightarrow \operatorname{Hom}_{\mathcal{E}}(-, Z) \rightarrow F \rightarrow 0 .
$$

Also, $\underline{\bmod }(\mathcal{C})$ denote the stable category of $\bmod (\mathcal{C})$ modulo projectives, and $\operatorname{Tr}: \underline{\bmod }(\mathcal{C}) \rightarrow \underline{\bmod }\left(\mathcal{C}^{\text {op }}\right)^{\text {op }}$ denotes the Auslander-Bridger transpose [10]. For a subcategory $\mathcal{X}$ of $\bmod (\mathcal{C})$ we let $\operatorname{Tr}(\mathcal{X})$ denote the subcategory of $\bmod \left(\mathcal{C}^{\mathrm{op}}\right)$ consisting of all functors $F$ which are isomorphic in $\underline{\bmod }\left(\mathcal{C}^{\mathrm{op}}\right)$ to $\operatorname{Tr}(F)$ for some $F \in \mathcal{X}$. For the notion of grade see Definition 5.7.

Theorem 1.6 (Theorem 5.8). Let $\mathcal{C}$ be an idempotent complete additive category. The association $\mathcal{E} \mapsto \bmod _{\mathrm{adm}}(\mathcal{E})$ gives a bijection between the following:

1. Exact structures $\mathcal{S}$ on $\mathcal{C}$, where $\mathcal{E}=(\mathcal{C}, \mathcal{S})$ is the corresponding exact category;

2. Subcategories $\mathcal{X}$ of $\bmod (\mathcal{C})$ satisfying the following:

(a) $\mathcal{X}$ is a resolving subcategory of $\mathcal{P}^{2}(\mathcal{C})$ and $\operatorname{Tr}(\mathcal{X})$ is a resolving subcategory of $\mathcal{P}^{2}\left(\mathcal{C}^{\mathrm{op}}\right)$;

(b) $\mathcal{X}$ and $\operatorname{Tr}(\mathcal{X})$ have no objects of grade 1 .

It was shown in $[25,30]$ that exact structures on an abelian category $\mathcal{A}$ correspond to closed subfunctors of $\operatorname{Ext}_{\mathcal{A}}^{1}(-,-)$. Such structures are one of the main objects of study in relative homological algebra $[31,32]$, see also $[2,3,4,5]$ for the case of Artin algebras. We obtain the following corollary for abelian categories.

Corollary 1.7 (Corollary 5.15). Let $\mathcal{A}$ be an abelian category. Then there exists a bijection between the following:

1. Exact structures on $\mathcal{A}$;

2. Resolving subcategories $\mathcal{X}$ of $\bmod (\mathcal{A})$ for which $\operatorname{Tr}(\mathcal{X})$ is a resolving subcategory of $\bmod \left(\mathcal{A}^{\mathrm{op}}\right)$.

\section{Structure of the paper}

In Section 2 we recall the quotients of exact categories that occur in the formulation of Auslander's formula. In particular, we show that localization by a two-sided admissible percolating subcategory reflects admissible morphisms, see Theorem 2.10. We end by investigating torsion theory on exact categories where the torsion part is a two-sided admissible percolating subcategory.

In Section 3 we study the category $\bmod _{a d m}(\mathcal{E})$. In particular, we show in Theorem 3.7 that it satisfies a universal property, in Theorem 3.9 that Auslander's formula holds, and in Corollary 3.12 that the association $\mathcal{E} \mapsto \bmod _{\text {adm }}(\mathcal{E})$ can be made into a left adjoint 2 -functor. We finish the section by showing that $\bmod _{\mathrm{adm}}(\mathcal{E})$ has the properties of an Auslander exact category.

In Section 4 we introduce Auslander exact categories, and we show that $\bmod _{a d m}(-)$ gives an equivalence onto the 2-category of Auslander exact categories, see Theorem 4.7. We then investigate how properties of $\mathcal{E}$ are reflected in $\bmod _{\text {adm }}(\mathcal{E})$, for example having enough injectives, see Theorem 4.21, and having enough projectives, see Proposition 4.25. We end by characterizing $\bmod _{\mathrm{adm}}(\mathcal{E})$ for Gorenstein projective modules, see Corollary 4.28, and for maximal Cohen-Macaulay modules, see Corollary 4.29.

In Section 5 we investigate different exact structures on an idempotent complete additive category by studying $\bmod _{a d m}(\mathcal{E})$. In particular, we show that $\bmod _{\mathrm{adm}}(\mathcal{E})$ is the smallest resolving subcategory of $\mathcal{P}^{2}(\mathcal{E})$ containing eff $(\mathcal{E})$, see Proposition 5.4. In the last part we show Theorem 1.6, which is the main result of the section. 


\section{Conventions}

All categories $\mathcal{C}$ are assumed to be essentially small. We also assume that they are additive, i.e. that $\operatorname{Hom}_{\mathcal{C}}(-,-)$ is enriched over abelian groups and that $\mathcal{C}$ admit finite direct sums. All subcategories are assumed to be full and closed under isomorphisms.

\section{Acknowledgements}

The authors thank Mikhail Gorsky for useful discussions and for providing some references. The 2nd author would like to thank the Hausdorff Institute for Mathematics in Bonn, since parts of the paper was written during his stay at the junior trimester program "New Trends in Representation Theory". The third authors is currently a postdoctoral researcher at FWO (12.M33.16N).

\section{Localizations/Quotients of exact categories}

This section is preliminary in nature, we establish basic conventions and terminology. We recall the notion of an exact category as introduced by Quillen in [56]. We recall the notion of a two-sided admissibly percolating subcategory as in [43] (these types of subcategories are called subcategories that localize in $[26])$. Given an exact category $\mathcal{E}$ and a two-sided admissibly percolating subcategory $\mathcal{A} \subseteq \mathcal{E}$, the quotient $\mathcal{E} / \mathcal{A}$ can be realized as a localization $\Sigma_{\mathcal{A}}^{-1} \mathcal{E}$.

\subsection{Exact categories}

Definition 2.1. $\quad$ 1. A conflation category is an additive category $\mathcal{C}$ together with a chosen class of kernel-cokernel pairs, called conflations, closed under isomorphisms. The kernel part of a conflation is called an inflation and the cokernel part of a conflation is called a deflation. We depict inflations by the symbol $\longmapsto$ and deflations by $\rightarrow$.

2. An additive functor $F: \mathcal{C} \rightarrow \mathcal{D}$ of conflation categories is called exact or conflation-exact if conflations are mapped to conflations.

3. A map $f: X \rightarrow Y$ in a conflation category $\mathcal{C}$ is called admissible if $f$ admits a factorization $X \rightarrow I \longmapsto Y$. Admissible morphisms are depicted by $X \stackrel{f}{\rightarrow} Y$.

4. A cochain $\cdots \rightarrow X^{n-1} \stackrel{d^{n-1}}{\longrightarrow} X^{n} \stackrel{d^{n}}{\longrightarrow} X^{n+1} \rightarrow \ldots$ in a conflation category $\mathcal{C}$ is called acyclic or exact if each $d^{i}$ is admissible and $\operatorname{ker}\left(d^{i+1}\right)=\operatorname{im}\left(d^{i}\right)$.

Remark 2.2. Note that if $f: X \rightarrow Y$ is admissible, the factorization $X \rightarrow I \mapsto Y$ is unique up to isomorphism. Moroever, $f$ admits a kernel and cokernel and $\operatorname{im}(f):=\operatorname{ker}(\operatorname{coker}(f)) \cong I \cong \operatorname{coker}(\operatorname{ker}(f))=$ : $\operatorname{coim}(f)$.

Definition 2.3. A conflation category $\mathcal{E}$ is called an exact category if $\mathcal{E}$ satisfies the following two dual lists of axioms:

R0 For any $X \in \mathcal{E}, X \rightarrow 0$ is a deflation.

R1 Deflations are closed under composition.

R2 Pullbacks along deflations exist, moreover, deflations are stable under pullbacks.

The dual list is given by:

L0 For any $X \in \mathcal{E}, 0 \mapsto X$ is an inflation.

L1 Inflations are closed under composition.

L2 Pushouts along inflations exist, moreover, inflations are stable under pushouts. 
Given an exact category $\mathcal{E}$, we let $\mathrm{K}^{\mathrm{b}}(\mathcal{E})$ denote the homotopy category of bounded complexes in $\mathcal{E}$, and we let $\mathbf{A} \mathbf{c}^{\mathrm{b}}(\mathcal{E})$ denote the subcategory of $\mathrm{K}^{\mathrm{b}}(\mathcal{E})$ consisting of the acyclic complexes. Note that $\mathbf{A} \mathbf{c}^{\mathrm{b}}(\mathcal{E})$ is not closed under isomorphism in $\mathrm{K}^{\mathrm{b}}(\mathcal{E})$ unless $\mathcal{E}$ is weakly idempotent complete, see [24, Proposition 10.14]. However, $\mathbf{A c}^{\mathrm{b}}(\mathcal{E})$ is still a triangulated subcategory of $\mathrm{K}^{\mathrm{b}}(\mathcal{E})$, see [24, Corollary 10.5]. The Verdier localization $\mathrm{K}^{\mathrm{b}}(\mathcal{E}) / \mathbf{A c}^{\mathrm{b}}(\mathcal{E})$ yields the triangulated category $\mathrm{D}^{\mathrm{b}}(\mathcal{E})$, called the bounded derived category of $\mathcal{E}$. The natural embedding $\mathcal{E} \hookrightarrow \mathrm{D}^{\mathrm{b}}(\mathcal{E})$, mapping objects to stalk complexes in degree zero, is fully faithful. Moreover, conflations correspond to triangles under this embedding.

\subsection{Localizations via two-sided admissibly percolating subcategories}

Throughout this section, let $\mathcal{E}$ be an exact category.

Definition 2.4. A full nonempty subcategory $\mathcal{A} \subseteq \mathcal{E}$ is called an admissibly deflation-percolating subcategory if the following axioms are satisfied:

A1 The category $\mathcal{A}$ is a Serre subcategory of $\mathcal{E}$, i.e. given a conflation $X \mapsto Y \rightarrow Z$ in $\mathcal{E}$, we have that $Y \in \mathcal{A} \Leftrightarrow X, Z \in \mathcal{A}$.

A2 All morphisms $f: X \rightarrow A$ with $A \in \mathcal{A}$ are admissible with image in $\mathcal{A}$, i.e. factors as

$$
X \rightarrow \operatorname{im}(f) \longmapsto A
$$

and $\operatorname{im}(f) \in \mathcal{A}$.

Dually, $\mathcal{A}$ is called an admissibly inflation-percolating subcategory if $\mathcal{A}^{\mathrm{op}}$ is an admissibly deflationpercolating subcategory in $\mathcal{E}^{\text {op }}$. Finally, $\mathcal{A}$ is a two-sided admissibly percolating subcategory if it is both an admissibly deflation and admissibly inflation-percolating subcategory.

Remark 2.5. 1. Since an admissibly deflation-percolating subcategory $\mathcal{A} \subseteq \mathcal{E}$ is closed under extensions, it inherits an exact structure from $\mathcal{E}$. It follows from axiom $\mathbf{A 2}$ that under this exact structure $\mathcal{A}$ becomes an abelian category.

2. Two-sided admissibly percolating subcategories are called localizing subcategories in [26].

The following definition is based on [64, Definition 1.12] and [26, Definition 4.0.36].

Definition 2.6. Let $\mathcal{A} \subseteq \mathcal{E}$ be an admissibly deflation-percolating subcategory. A morphism $f: X \rightarrow Y$ in $\mathcal{E}$ is called a weak $\mathcal{A}$-isomorphism or simply a weak isomorphism if $\mathcal{A}$ is understood, if $f$ is admissible and $\operatorname{ker}(f), \operatorname{coker}(f) \in \mathcal{A}$. Morphisms endowed with $\sim$ are weak isomorphisms. The set of weak isomorphisms is denoted by $\Sigma_{\mathcal{A}}$.

Proposition 2.7. Let $\mathcal{A} \subseteq \mathcal{E}$ be an admissibly deflation-percolating subcategory.

1. The set $\Sigma_{\mathcal{A}}$ is a right multiplicative system.

2. The set $\Sigma_{\mathcal{A}}$ is saturated, i.e. $Q(f)$ is an isomorphism in $\Sigma_{\mathcal{A}}^{-1} \mathcal{E}$ if and only if $f \in \Sigma_{\mathcal{A}}$.

3. If $Q(M) \cong 0$ for an object $M \in \mathcal{E}$, then $M \in \mathcal{A}$.

4. Pullbacks along weak isomorphisms exist, moreover, weak isomorphisms are stable under pullbacks.

5. Admissible morphisms are stable under pullbacks along weak isomorphisms.

Proof. We refer the reader to [43] for the first four statements. The last statement follows from [43, Corollary 6.10] and the fact that deflations are preserved under pullbacks.

The following theorem yields that the localization $\Sigma_{\mathcal{A}}^{-1} \mathcal{E}$ satisfies the universal property of the quotient $\mathcal{E} / \mathcal{A}$.

Theorem 2.8. Let $\mathcal{A} \subseteq \mathcal{E}$ be a two-sided admissibly percolating subcategory and write $Q: \mathcal{E} \rightarrow \Sigma_{\mathcal{A}}^{-1} \mathcal{E}$ for the localization functor. 
1. $Q$ sends conflations in $\mathcal{E}$ to kernel-cokernel pairs in $\Sigma_{\mathcal{A}}^{-1} \mathcal{E}$. Furthermore, closing this class of kernelcokernel pairs under isomorphism makes $\Sigma_{\mathcal{A}}^{-1} \mathcal{E}$ into an exact category.

2. The functor $Q$ satisfies the 2-universal property of a quotient $\mathcal{E} / \mathcal{A}$ in the category of exact categories, i.e. for any exact category $\mathcal{F}$ and exact functor $F: \mathcal{E} \rightarrow \mathcal{F}$ such that $F(\mathcal{A}) \cong 0$, there exists an exact functor $F^{\prime}: \Sigma_{\mathcal{A}}^{-1} \mathcal{E} \rightarrow \mathcal{F}$ such that $F^{\prime} \circ Q=F$.

3. The localization sequence $\mathcal{A} \rightarrow \mathcal{E} \stackrel{Q}{\rightarrow} \Sigma_{\mathcal{A}}^{-1} \mathcal{E}$ induces the Verdier localization sequence

$$
\mathrm{D}_{\mathcal{A}}^{\mathrm{b}}(\mathcal{E}) \rightarrow \mathrm{D}^{\mathrm{b}}(\mathcal{E}) \rightarrow \mathrm{D}^{\mathrm{b}}(\mathcal{E} / \mathcal{A})
$$

where $\mathrm{D}_{\mathcal{A}}^{\mathrm{b}}(\mathcal{E})$ is the thick full triangulated subcategory of $\mathrm{D}^{\mathrm{b}}(\mathcal{E})$ generated by $\mathcal{A}$ under the canonical embedding $\mathcal{E} \hookrightarrow \mathrm{D}^{\mathrm{b}}(\mathcal{E})$.

Explicitly, a sequence $X \stackrel{f}{\rightarrow} Y \stackrel{g}{\rightarrow} Z$ in $\Sigma_{\mathcal{A}}^{-1} \mathcal{E}$ is a conflation if and only if there exists a commutative diagram

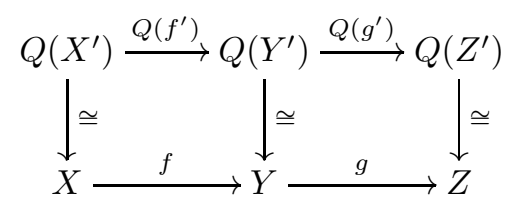

where the vertical maps are isomorphisms in $\Sigma_{\mathcal{A}}^{-1} \mathcal{E}$, and where $X^{\prime} \stackrel{f^{\prime}}{\rightarrow} Y^{\prime} \stackrel{g^{\prime}}{\rightarrow} Z^{\prime}$ is a conflation in $\mathcal{E}$.

\subsection{Admissible morphisms in $\mathcal{E} / \mathcal{A}$}

In this section we show that the localization functor $Q: \mathcal{E} \rightarrow \mathcal{E} / \mathcal{A}$ reflects admissible morphisms when $\mathcal{A}$ is a two-sided admissibly percolating subcategory.

Proposition 2.9. Let $\mathcal{E}$ be an exact category, and let $\mathcal{A}$ be an admissibly deflation-percolating subcategory of $\mathcal{E}$. Let $f: X \rightarrow Y$ and $g: Y \rightarrow Z$ be morphisms in $\mathcal{E}$. The following holds:

1. If $f$ is an inflation with cokernel in $\mathcal{A}$ and $g$ is a deflation, then $g \circ f=f^{\prime} \circ g^{\prime}$ where $g^{\prime}$ is a deflation and $f^{\prime}$ is an inflation with cokernel in $\mathcal{A}$;

2. If $f$ is a weak isomorphism and $g$ is admissible, then $g \circ f$ is admissible;

3. If $g$ is a weak isomorphism and $g \circ f$ is admissible, then $f$ is admissible.

Proof. 1. This follows from [43, Corollary 6.10].

2. Write $f=f_{2} \circ f_{1}$ and $g=g_{2} \circ g_{1}$ where $f_{1}, g_{1}$ are deflations and $f_{2}, g_{2}$ are inflations. By (1) we have that $g_{1} \circ f_{2}$ can be written as a composite $f_{2}^{\prime} \circ g_{1}^{\prime}$ where $g_{1}^{\prime}$ is a deflation and $f_{2}^{\prime}$ is an inflation. In particular, $g_{1} \circ f_{2}$ is admissible. Hence the composite $g \circ f=g_{2} \circ\left(g_{1} \circ f_{2}\right) \circ f_{1}$ is admissible, which proves the claim.

3. Taking the pullback of $g$ along $g \circ f$, we get a commutative diagram

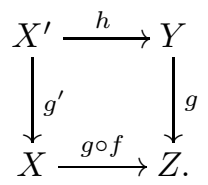

Since $g \circ f: X \rightarrow Z$ factors through $g: Y \rightarrow Z$ via $f$, there exists a map $i: X \rightarrow X^{\prime}$ satisfying $g^{\prime} \circ i=1_{X}$ and $h \circ i=f$. Since weak isomorphisms are preserved by pullbacks, $g^{\prime}$ must be a weak isomorphism, and hence a deflation since it has a right inverse. Therefore, $i$ is an inflation with cokernel in $\mathcal{A}$. Also, since admissible morphisms are preserved under pullbacks by weak isomorphisms, $h$ must be admissible. Since $f=h \circ i$, we get that $f$ is admissible by the first part of the proof 
Theorem 2.10. A map $f: X \rightarrow Y$ is admissible in $\mathcal{E}$ if and only if $Q(f)$ is admissible in $\mathcal{E} / \mathcal{A}$. In other words, the localization functor $Q$ reflects admissible morphisms.

Proof. As $Q(f)$ is admissible in $\Sigma_{\mathcal{A}}^{-1} \mathcal{E}$, there is a deflation $A \rightarrow B$ and an inflation $C \longmapsto D$ in $\mathcal{E}$ and isomorphisms $B \cong C, A \cong X$ and $D \cong Y$ in $\Sigma_{\mathcal{A}}^{-1} \mathcal{E}$ making the diagram



commutative in $\Sigma_{\mathcal{A}}^{-1} \mathcal{E}$. Let $B \stackrel{\sim}{\rightarrow} B^{\prime} \stackrel{\sim}{\leftarrow} C$ be a roof in $\mathcal{E}$ representing the isomorphism $B \cong C$, (by Proposition 2.7.(2), $\Sigma_{\mathcal{A}}$ is saturated and thus both arrows in this roof belong to $\Sigma_{\mathcal{A}}$ ). Taking the pushout $D^{\prime}$ of $C \longmapsto D$ along the weak isomorphism $C \stackrel{\sim}{\rightarrow} B^{\prime}$ yields an inflation $B^{\prime} \longmapsto D^{\prime}$ in $\mathcal{E}$ and an isomorphism $D^{\prime} \cong D$ in $\Sigma_{\mathcal{A}}^{-1} \mathcal{E}$ by the dual of Proposition 2.7.(5). The composition $A \rightarrow B \stackrel{\sim}{\rightarrow} B^{\prime} \longmapsto D^{\prime}$ is admissible as $B \stackrel{\sim}{\rightarrow} B^{\prime}$ is admissible. Thus, we have a commutative diagram

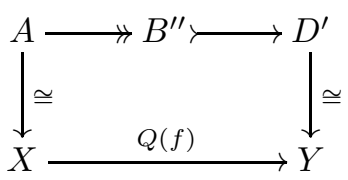

in $\Sigma_{\mathcal{A}}^{-1} \mathcal{E}$

Since $A \cong X$, there is a roof $X \stackrel{\sim}{\rightarrow} \widetilde{\leftarrow} A$ in $\mathcal{E}$ representing this map. By the dual of Proposition 2.7.(4) and Proposition 2.7.(5), taking the pushout of the admissible map $A \rightarrow B^{\prime \prime} \longmapsto D^{\prime}$ along the weak isomorphism $A \stackrel{\sim}{\rightarrow} \bar{X}$, we obtain an admissible map $\bar{X} \rightarrow \bar{B} \mapsto \bar{D}$ with $\bar{D} \cong D^{\prime}$ in $\Sigma_{\mathcal{A}}^{-1} \mathcal{E}$. By Proposition 2.9. (2), the composition $X \stackrel{\sim}{\rightarrow} \bar{X} \rightarrow \bar{B}$ is admissible and thus factors as $X \rightarrow U \mapsto \bar{B}$. By axiom L1, the composition $U \longmapsto \bar{B} \longmapsto \bar{D}$ is an inflation. Hence, we have the commutative diagram



in $\Sigma_{\mathcal{A}}^{-1} \mathcal{E}$

As $\bar{D} \cong Y$ in $\Sigma_{\mathcal{A}}^{-1} \mathcal{E}$, there is a roof $\bar{D} \stackrel{\sim}{\rightarrow} \bar{Y} \stackrel{\sim}{\leftarrow}$ in $\mathcal{E}$ representing this map. Clearly, the compositions $X \stackrel{f}{\rightarrow} Y \stackrel{\sim}{\rightarrow} \bar{Y}$ and $X \rightarrow U \longmapsto \bar{D} \stackrel{\sim}{\rightarrow} \bar{Y}$ are equal in $\Sigma_{\mathcal{A}}^{-1} \mathcal{E}$. As $\Sigma_{\mathcal{A}}$ is a left multiplicative system, there exists a weak isomorphism $\bar{Y} \rightarrow Z$ such that the compositions $X \stackrel{f}{\rightarrow} Y \stackrel{\sim}{\rightarrow} \bar{Y} \stackrel{\sim}{\rightarrow} Z$ and $X \rightarrow U \mapsto \bar{D} \stackrel{\sim}{\rightarrow} \bar{Y} \stackrel{\sim}{\rightarrow} Z$ are equal in $\mathcal{E}$. Since the latter composition is admissible by the dual of Proposition 2.9. (2), it follows that $X \stackrel{f}{\rightarrow} Y \stackrel{\sim}{\rightarrow} \bar{Y} \stackrel{\sim}{\rightarrow} Z$ is admissible. Finally, by Proposition 2.9. (3) we get that $f$ is admissible.

\subsection{Torsion theory with two-sided admissibly percolating subcategories}

In this section we show that for any torsion pair $(\mathcal{T}, \mathcal{F})$ in an exact category $\mathcal{E}$ such that $\mathcal{T}$ satisfies axiom A2, $\mathcal{T} \subseteq \mathcal{E}$ is a two-sided admissibly percolating subcategory. Moreover, the torsion and torsionfree functor enjoy further properties.

Recall the notion of a torsion theory in an exact category.

Definition 2.11. Let $\mathcal{E}$ be an exact category. A torsion theory or torsion pair is a pair $(\mathcal{T}, \mathcal{F})$ of full subcategories closed under isomorphism such that

1. $\operatorname{Hom}_{\mathcal{E}}(T, F)=0$ for all $T \in \mathcal{T}$ and $F \in \mathcal{F}$;

2. for any object $M \in \mathcal{E}$, there exists a conflation $T \longmapsto M \rightarrow F$ with $T \in \mathcal{T}$ and $F \in \mathcal{F}$. 
For a subcategory $\mathcal{X}$ of $\mathcal{E}$ we let ${ }^{\perp} \mathcal{X}$ and $\mathcal{X}^{\perp}$ denote the subcategories consisting of all objects $E \in \mathcal{E}$ satisfying $\operatorname{Hom}_{\mathcal{E}}(E, X)=0$ or $\operatorname{Hom}_{\mathcal{E}}(X, E)=0$ for all $X \in \mathcal{X}$, respectively. The following proposition is standard (see for example [54]).

Proposition 2.12. Let $(\mathcal{T}, \mathcal{F})$ be a torsion pair in an exact category $\mathcal{E}$.

1. $\mathcal{T}={ }^{\perp} \mathcal{F}$ and $\mathcal{F}=\mathcal{T}^{\perp}$;

2. The subcategory $\mathcal{T} \subseteq \mathcal{E}$ is closed under extensions and epimorphic quotients. Dually, the subcategory $\mathcal{F} \subseteq \mathcal{E}$ is closed under extensions and subobjects. In particular, $\mathcal{T}$ and $\mathcal{F}$ inherit exact structures from $\mathcal{E}$.

3. For each $M \in \mathcal{E}$, there is a unique (up to isomorphism) conflation $\mathfrak{t} M \longmapsto M \rightarrow \mathfrak{f} M$ with $\mathfrak{t} M \in \mathcal{T}$ and $\mathfrak{f} M \in \mathcal{F}$.

4. The inclusion functor $\mathcal{T} \rightarrow \mathcal{E}$ has right adjoint $\mathfrak{t}$ such that $\mathfrak{t}(M)=\mathfrak{t} M$ as above. Dually, the inclusion functor $\mathcal{F} \rightarrow \mathcal{E}$ has a left adjoint $\mathfrak{f}$ such that $\mathfrak{f}(M)=\mathfrak{f} M$.

The following definition is adapted from [54].

Definition 2.13. An additive functor $F: \mathcal{E} \rightarrow \mathcal{F}$ between exact categories is called left exact if a conflation $X \stackrel{f}{\longmapsto} Y \stackrel{g}{\rightarrow} Z$ is mapped to an exact sequence $F(X) \stackrel{F(f)}{\longrightarrow} F(Y) \stackrel{F(g)}{\longrightarrow} F(Z) \longrightarrow \operatorname{coker}(F(g))$. The notion of a right exact functor is defined dually.

Remark 2.14. If $F: \mathcal{E} \rightarrow \mathcal{F}$ is left exact and $h: Y \rightarrow Z$ is an admissible morphism in $\mathcal{E}$ with kernel $f: X \longmapsto Y$, then $F(h): F(Y) \rightarrow F(Z)$ is an admissible morphism in $\mathcal{F}$ with kernel $F(f): F(X) \longmapsto F(Y)$. Indeed, choosing a factorization $Y \stackrel{g^{\prime}}{\rightarrow} \operatorname{im}(f) \stackrel{g^{\prime \prime}}{\longmapsto} Z$ of $g$ and applying $F$ to the conflation $X \stackrel{f}{\longmapsto} Y \stackrel{g^{\prime}}{\rightarrow} \operatorname{im}(f)$ we get an admissible morphism $F\left(g^{\prime}\right): F(Y) \rightarrow F(\operatorname{im}(f))$ with kernel $F(f): F(X) \longmapsto F(Y)$. The claim now follows since $F(g)=F\left(g^{\prime \prime}\right) \circ F\left(g^{\prime}\right)$ and $F\left(g^{\prime \prime}\right)$ is an inflation.

Remark 2.15. Note that a left exact (resp right exact) functor between exact categories does not necessarily preserve all kernels (resp cokernels), but only kernels of admissible morphisms.

Proposition 2.16. Let $\mathcal{E}$ be an exact category with a torsion pair $(\mathcal{T}, \mathcal{F})$ such that $\mathcal{T} \subseteq \mathcal{E}$ satisfies axiom A2.

1. The subcategory $\mathcal{T} \subseteq \mathcal{E}$ is two-sided admissibly percolating.

2. The functor $\mathfrak{t}$ is left exact and the functor $\mathfrak{f}$ preserves inflations and deflations.

3. The functor $f$ reflects admissible morphisms.

Proof. 1. We first show axiom $\mathbf{A} 2^{\text {op }}$. Let $f: T \rightarrow X$ be a map with $X \in \mathcal{T}$. As $(\mathcal{T}, \mathcal{F})$ is a torsion pair, there is a conflation $\mathfrak{t} X \longmapsto X \rightarrow \mathfrak{f} X$. Note that $f$ factors through $\mathfrak{t} X \longmapsto X$ as $\operatorname{Hom}(\mathcal{T}, \mathcal{F})=0$. By axiom A2, the map $T \rightarrow \mathfrak{t} X$ is admissible with image in $\mathcal{T}$. It follows that the composition $T \rightarrow \mathfrak{t} X \longmapsto X$ is admissible with image in $\mathcal{T}$. This shows axiom $\mathbf{A 2}^{\text {op }}$.

We now show axiom A1. Let $X \mapsto Y \rightarrow Z$ be a conflation in $\mathcal{E}$. Assume first that $Y \in \mathcal{T}$. By axiom A2, the map $X \longmapsto Y$ is admissible with image in $\mathcal{T}$ and thus $X \in \mathcal{T}$. Similarly, by axiom $\mathbf{A 2}^{\text {op }}$ yields that $Y \rightarrow Z$ is admissible with image in $\mathcal{T}$ and thus $Z \in \mathcal{T}$. Conversely, if $X, Z \in \mathcal{T}$, then $Y \in \mathcal{T}$ as $\mathcal{T} \subseteq \mathcal{E}$ is extension-closed.

2. Let $X \stackrel{a}{\longmapsto} Y \stackrel{b}{\rightarrow} Z$ be a conflation in $\mathcal{E}$. By axiom $\mathbf{A} 2$ the maps $\mathfrak{t}(a)$ and $\mathfrak{t}(b)$ are admissible. As $\mathfrak{t}(a)$ is monic, it is an inflation. Since $\mathfrak{t}$ is a right adjoint, $\mathfrak{t}$ commutes with kernels, and thus the kernel of $\mathfrak{t}(b)$ in $\mathcal{T}$ is given by $\mathfrak{t}(a)$. Since $\mathcal{T}$ is abelian and the inclusion $\mathcal{T} \rightarrow \mathcal{E}$ is exact, it follows that $\mathfrak{t}(a)$ is also the kernel of $\mathfrak{t}(b)$ in $\mathcal{E}$. This shows that $\mathfrak{t}$ is left exact. In particular, it follows that 
$\operatorname{im}(\mathfrak{t}(b)) \cong \operatorname{coker}(\mathfrak{t}(a))$. By axiom $\mathbf{L} 1$, the composition $\operatorname{im}(\mathfrak{t}(b)) \longmapsto \mathfrak{t} Z \longmapsto Z$ is an inflation and we write $L$ for its cokernel. Consider the following two commutative diagrams:
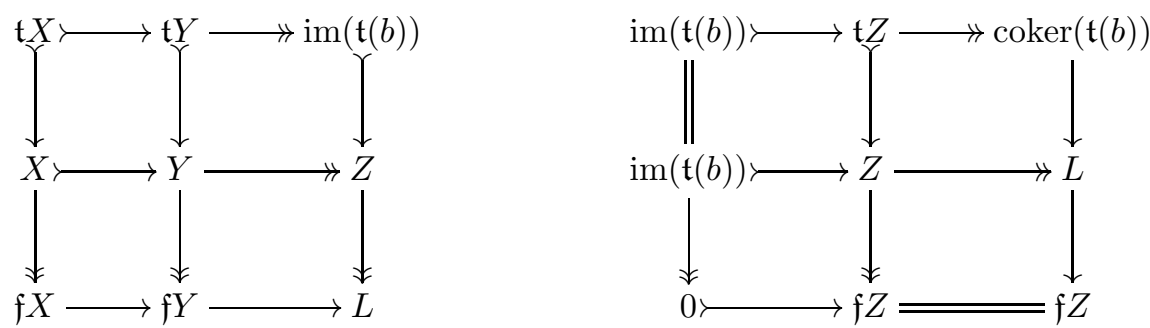

Applying the Nine Lemma to both diagrams, we obtain conflations

$$
\mathfrak{f} X \mapsto \mathfrak{f} Y \rightarrow L \quad \text { and } \quad \operatorname{coker}(\mathfrak{t}(b)) \longmapsto L \rightarrow \mathfrak{f} Z .
$$

By axiom $\mathbf{R} 1$, the composition $\mathfrak{f} Y \rightarrow L \rightarrow f Z$ is a deflation. The result follows.

3. Let $f: X \rightarrow Y$ be a morphism in $\mathcal{E}$. Consider the following commutative diagram:

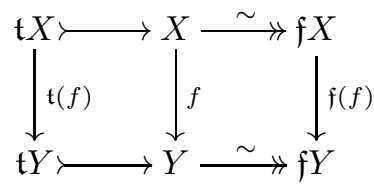

If $f$ is admissible, then $\mathfrak{f}(f)$ is admissible as $\mathfrak{f}$ preserves inflations and deflations. Conversely, if $\mathfrak{f}(f)$ is admissible, then the composition $X \rightarrow \mathfrak{f} X \stackrel{\mathfrak{f}(f)}{\longrightarrow} \mathfrak{f} Y$ is admissible. Therefore the composition $X \stackrel{f}{\rightarrow} Y \rightarrow \mathfrak{f} Y$ is admissible, and hence $f$ is admissible by Proposition 2.9.(3).

Remark 2.17. Despite the functor $\mathfrak{f}$ preserving inflations and deflations, it need not be conflation-exact. Indeed, consider the standard torsion theory on the category abelian groups. The short exact sequence $\mathbb{Z} \stackrel{\cdot n}{\mapsto} \mathbb{Z} \rightarrow \mathbb{Z} / n \mathbb{Z}$ is mapped to $\mathbb{Z} \stackrel{\cdot n}{\rightarrow} \mathbb{Z} \rightarrow 0$.

\section{Auslander's formula for exact categories}

Given an exact category $\mathcal{E}$, we consider the category $\bmod _{\text {adm }}(\mathcal{E})$ of admissibly presented functors and the subcategory $\operatorname{eff}(\mathcal{E}) \subseteq \bmod _{\text {adm }}(\mathcal{E})$ of effaceable functors (see Definition 3.1 below). We show that Auslander's formula extends to exact categories, i.e. the categories $\mathcal{E}$ and $\bmod _{\text {adm }}(\mathcal{E}) / \mathrm{eff}(\mathcal{E})$ are equivalent as exact categories. Moreover, $\operatorname{eff}(\mathcal{E}) \subseteq \bmod _{\text {adm }}(\mathcal{E})$ is a two-sided admissibly percolating subcategory that arises as the torsion part of a torsion theory on $\bmod _{\text {adm }}(\mathcal{E})$.

\subsection{Basic definitions and results}

Write $\Upsilon: \mathcal{E} \rightarrow \operatorname{Mod}(\mathcal{E}): A \mapsto \operatorname{Hom}_{\mathcal{E}}(-, A)$ for the Yoneda embedding . We start with the following definition.

Definition 3.1. Let $\mathcal{E}$ be a conflation category.

1. Let $\bmod (\mathcal{E})$ be the full subcategory of $\operatorname{Mod}(\mathcal{E})$ consisting of those contravariant functors $F: \mathcal{E} \rightarrow \mathrm{Ab}$ that admit a projective representation

$$
\Upsilon(X) \stackrel{\Upsilon(f)}{\longrightarrow} \Upsilon(Y) \rightarrow F \rightarrow 0 .
$$

2. Let $\bmod _{a d m}(\mathcal{E})$ be the full subcategory of $\operatorname{Mod}(\mathcal{E})$ consisting of those functors $F$ that admit a projective representation

$$
\Upsilon(X) \stackrel{\Upsilon(f)}{\longrightarrow} \Upsilon(Y) \rightarrow F \rightarrow 0
$$

such that $f: X \rightarrow Y$ is an admissible morphism in $\mathcal{E}$. We refer to $f$ as a underlying or a representing morphism of $F$. 
3. Let $\operatorname{eff}(\mathcal{E})$ be the full subcategory of $\operatorname{Mod}(\mathcal{E})$ consisting of those functors $F$ that admit a projective representation

$$
\Upsilon(X) \stackrel{\Upsilon(f)}{\longrightarrow} \Upsilon(Y) \rightarrow F \rightarrow 0
$$

such that $f: X \rightarrow Y$ is a deflation in $\mathcal{E}$.

4. Let $\mathcal{F}$ be the full subcategory of $\operatorname{Mod}(\mathcal{E})$ consisting of those functors $F$ that admit a projective representation

$$
\Upsilon(X) \stackrel{\Upsilon(f)}{\longrightarrow} \Upsilon(Y) \rightarrow F \rightarrow 0
$$

such that $f: X \longmapsto Y$ is an inflation in $\mathcal{E}$.

Lemma 3.2. Every $F \in \bmod _{\mathrm{adm}}(\mathcal{E})$ fits into an exact sequence

$$
0 \rightarrow \Upsilon(\operatorname{ker}(f)) \rightarrow \Upsilon(X) \stackrel{\Upsilon(f)}{\longrightarrow} \Upsilon(Y) \rightarrow F \rightarrow 0 .
$$

in $\operatorname{Mod}(\mathcal{E})$.

Proof. The Yoneda embedding $\Upsilon: \mathcal{E} \rightarrow \operatorname{Mod}(\mathcal{E})$ is a left exact covariant functor. The result follows as $f$ admits a kernel.

The following proposition will be used multiple times throughout the text.

Proposition 3.3. Let $\mathcal{C}$ be an additive category and write $\Upsilon: \mathcal{C} \rightarrow \operatorname{Mod}(\mathcal{C})$ for the Yoneda embedding. Consider a commutative diagram

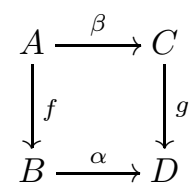

in $\mathcal{E}$ such that $g$ admits a kernel $k: \operatorname{ker}(g) \rightarrow C$ and such that the cospan $B \stackrel{\alpha}{\rightarrow} D \stackrel{g}{\leftarrow} C$ admits a pullback E. Write $F=\operatorname{coker}(\Upsilon(f)), G=\operatorname{coker}(\Upsilon(g))$ and $\eta: F \rightarrow G$ for the induced map. Consider the commutative diagram



where ECBD is a pullback square and $\beta=\beta^{\prime} \beta^{\prime \prime}$. Applying $\Upsilon$ and taking the cokernel of the vertical maps induces the epi-mono factorization

$$
\operatorname{ker}(\eta) \longleftrightarrow F \longrightarrow \operatorname{im}(\eta) \longleftrightarrow G \longrightarrow \operatorname{coker}(\eta)
$$

of $\eta$ in $\operatorname{Mod}(\mathcal{C})$.

Proof. This is a standard diagram chase argument.

\subsection{The quotient $\bmod _{\mathrm{adm}}(\mathcal{E}) / \operatorname{eff}(\mathcal{E})$}

Throughout this section, $\mathcal{E}$ denotes an exact category.

Proposition 3.4. The category $\bmod _{a d m}(\mathcal{E})$ is an extension-closed subcategory of $\operatorname{Mod}(\mathcal{E})$. In particular, $\bmod _{\mathrm{adm}}(\mathcal{E})$ inherits an exact structure from $\operatorname{Mod}(\mathcal{E})$. 
Proof. Let $0 \rightarrow F \rightarrow G \rightarrow H \rightarrow 0$ be a short exact sequence in the abelian category $\operatorname{Mod}(\mathcal{E})$ and assume that $F, H \in \bmod _{\text {adm }}(\mathcal{E})$. Let $\Upsilon(\operatorname{ker}(f)) \stackrel{\Upsilon\left(k_{f}\right)}{\longrightarrow} \Upsilon(A) \stackrel{\Upsilon(f)}{\longrightarrow} \Upsilon(B) \rightarrow F$ and $\Upsilon(\operatorname{ker}(h)) \stackrel{\Upsilon\left(k_{h}\right)}{\longrightarrow} \Upsilon(C) \stackrel{\Upsilon(h)}{\longrightarrow}$ $\Upsilon(D) \rightarrow H$ be projective resolutions of $F$ and $H$ respectively with $f, h$ admissible morphisms. By the Horseshoe Lemma and the fact that the Yoneda embedding is faithful, one finds a projective resolution $\Upsilon(\operatorname{ker}(h) \oplus \operatorname{ker}(f)) \stackrel{\Upsilon\left(\begin{array}{cc}k_{h} & 0 \\ l^{\prime} & k_{f}\end{array}\right)}{\longrightarrow} \Upsilon(C \oplus A) \stackrel{\Upsilon\left(\begin{array}{ll}h & 0 \\ l & f\end{array}\right)}{\longrightarrow} \Upsilon(D \oplus B) \rightarrow G$ for some morphisms $l: C \rightarrow B$ and $l^{\prime}: \operatorname{ker}(h) \rightarrow A$. Let $C \stackrel{h^{\prime}}{\rightarrow} \operatorname{im}(h) \stackrel{h^{\prime \prime}}{\longrightarrow}$ and be a factorization of $h$ into a deflation and an inflation. We obtain the following morphism of acyclic complexes in $\mathcal{E}$ :



By [53, Lemma 1.1], the cone is an acyclic complex as well. It follows that the map $\left(\begin{array}{cc}h^{\prime} & 0 \\ l & f\end{array}\right): C \oplus A \rightarrow$ $\operatorname{im}(h) \oplus B$ is admissible. Also, the map $\left(\begin{array}{cc}h^{\prime \prime} & 0 \\ 0 & 1\end{array}\right): \operatorname{im}(h) \oplus B \rightarrow D \oplus B$ is an inflation as the direct sum of inflations are inflations. It follows that $\left(\begin{array}{ll}h & 0 \\ l & f\end{array}\right)=\left(\begin{array}{cc}h^{\prime \prime} & 0 \\ 0 & 1_{B}\end{array}\right)\left(\begin{array}{cc}h^{\prime} & 0 \\ l & f\end{array}\right)$ is admissible. This shows that $G \in \bmod _{\text {adm }}(\mathcal{E})$.

Proposition 3.5. The pair $(\operatorname{eff}(\mathcal{E}), \mathcal{F})$ is a torsion pair in $\bmod _{\text {adm }}(\mathcal{E})$ such that $\operatorname{eff}(\mathcal{E}) \subseteq \bmod _{\text {adm }}(\mathcal{E})$ satisfies axiom A2. In particular, $\mathrm{eff}(\mathcal{E}) \subseteq \bmod _{\mathrm{adm}}(\mathcal{E})$ is a two-sided admissibly percolating subcategory.

Proof. We first show that $(\operatorname{eff}(\mathcal{E}), \mathcal{F})$ yields a torsion pair in $\bmod _{\text {adm }}(\mathcal{E})$. To that end, let $G \in \bmod _{\text {adm }}(\mathcal{E})$ and choose a projective presentation $\Upsilon(X) \stackrel{\Upsilon(f)}{\longrightarrow} \Upsilon(Y) \rightarrow G \rightarrow 0$ where $f$ is admissible. Let $X \stackrel{f^{\prime}}{\rightarrow} X^{\prime} \stackrel{f^{\prime \prime}}{\longmapsto}$ $Y$ be the deflation-inflation factorization of $f$. The commutative diagram

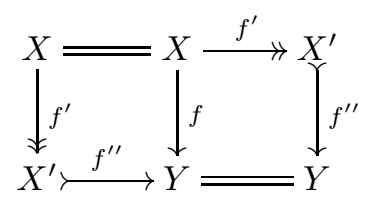

in $\mathcal{E}$ induces a sequence $F \stackrel{\phi}{\rightarrow} G \stackrel{\psi}{\rightarrow} H$ in $\bmod _{\text {adm }}(\mathcal{E})$ where $F=\operatorname{coker}\left(\Upsilon\left(f^{\prime}\right)\right) \in \operatorname{eff}(\mathcal{E})$ and $H=$ $\operatorname{coker}\left(\Upsilon\left(f^{\prime \prime}\right)\right) \in \mathcal{F}$. A straightforward diagram chase shows that $F \stackrel{\phi}{\rightarrow} G \stackrel{\psi}{\rightarrow} H$ is a short exact sequence.

We now show that $\operatorname{Hom}_{\bmod _{\text {adm }}(\mathcal{E})}(\operatorname{eff}(\mathcal{E}), \mathcal{F})=0$. Let $\eta: F \rightarrow H$ be a morphism in $\bmod _{\text {adm }}(\mathcal{E})$ with $F \in \operatorname{eff}(\mathcal{E})$ and $H \in \mathcal{F}$. Choose projective presentations $\Upsilon(X) \stackrel{\Upsilon(f)}{\longrightarrow} \Upsilon(Y) \rightarrow F \rightarrow 0$ and $\Upsilon\left(X^{\prime}\right) \stackrel{\Upsilon(h)}{\longrightarrow}$ $\Upsilon\left(Y^{\prime}\right) \rightarrow H \rightarrow 0$ where $f$ is a deflation and $h$ is an inflation in $\mathcal{E}$. Lifting the morphism $\eta$ to a map between the projective presentations and using that the Yoneda embedding is fully faithful, we find morphisms $\alpha: X \rightarrow X^{\prime}$ and $\beta: Y \rightarrow Y^{\prime}$ such that $h \circ \alpha=\beta \circ f$ and making the diagram



commutative. Moreover, using Lemma 3.2, we find that $\alpha \circ k_{f}=0$ (where $k_{f}: \operatorname{ker}(f) \longmapsto X$ is the kernel of $f$ ). Since $h \circ \alpha \circ k_{f}=\beta \circ f \circ k_{f}=0$ and $h$ is an inflation, it follows that $\alpha \circ k_{f}=0$. Hence $\alpha$ factors through $f$ via a morphism $\gamma: Y \rightarrow X^{\prime}$. Since $h \circ \gamma \circ f=h \circ \alpha=\beta \circ f$ and $f$ is a deflation, we get that $h \circ \gamma=\beta$. Hence $\Upsilon(\beta)$ factors through $\Upsilon(h)$, so $\eta$ must be 0 .

To prove that $\operatorname{eff}(\mathcal{E}) \subseteq \bmod _{\text {adm }}(\mathcal{E})$ satisfies axiom A2, assume we are given a morphism $\eta: F \rightarrow G$ with $G \in \bmod _{\text {adm }}(\mathcal{E})$ and $F \in \operatorname{eff}(\mathcal{E})$. Choose projective presentations $\Upsilon(A) \stackrel{\Upsilon(f)}{\longrightarrow} \Upsilon(B) \rightarrow F \rightarrow 0$ and 
$\Upsilon(C) \stackrel{\Upsilon(g)}{\longrightarrow} \Upsilon(D) \rightarrow G \rightarrow 0$ where $f$ is admissible and $g$ is a deflation. Lifting $\eta$ to these projective presentation gives maps $\alpha: B \rightarrow D$ and $\beta: A \rightarrow C$ making the square

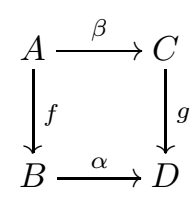

commutative. By taking the pullback of $\alpha$ along $g$ we get the diagram (1) in Proposition 3.3, which induces the factorization

$$
\operatorname{ker}(\eta) \longleftrightarrow F \longrightarrow \operatorname{im}(\eta) \longrightarrow G \longrightarrow \operatorname{coker}(\eta)
$$

of $\eta$ in $\operatorname{Mod}(\mathcal{E})$. Since $g^{\prime}$ and $(g \alpha)$ are deflations, it follows that $\operatorname{im}(\eta)$ and $\operatorname{coker}(\eta)$ are in $\operatorname{eff}(\mathcal{E})$. Now let $A \stackrel{f^{\prime}}{\rightarrow} B^{\prime} \stackrel{f^{\prime \prime}}{\hookrightarrow} B$ be a deflation-inflation factorization of $f$. Taking the pullback $E^{\prime}$ of $f^{\prime \prime}$ along $g^{\prime}$, we get a commutative diagram



where the rows are exact and where the composite $A \rightarrow E^{\prime} \longmapsto E$ is equal to $\beta^{\prime \prime}$. Since the pullback of an inflation along a deflation is an inflation [24, Proposition 2.15], the map $E^{\prime} \longmapsto E$ is an inflation. Also, by [24, Proposition 2.12] the top left square is pushout square and the induced map $\operatorname{ker}(g) \oplus A \rightarrow E^{\prime}$ is a deflation. Since $\left(k^{\prime} \beta^{\prime \prime}\right): \operatorname{ker}(g) \oplus A \rightarrow E$ is equal to the composite $\operatorname{ker}(g) \oplus A \rightarrow E^{\prime} \longmapsto E$, it must be admissible. Hence $\operatorname{ker}(\eta) \in \bmod _{\text {adm }}(\mathcal{E})$, which proves axiom A2. Finally, by Proposition 2.16, eff $(\mathcal{E}) \subseteq$ $\bmod _{a d m}(\mathcal{E})$ is a two-sided admissibly percolating subcategory.

Corollary 3.6. The quotient $\bmod _{\mathrm{adm}}(\mathcal{E}) / \mathrm{eff}(\mathcal{E})$ is an exact category.

\subsection{Auslander's formula for exact categories}

In this section $\mathcal{E}$ denotes an exact category.

Theorem 3.7 (Universal property). $\quad$ 1. The Yoneda embedding $\Upsilon: \mathcal{E} \rightarrow \bmod _{\text {adm }}(\mathcal{E})$ is left exact;

2. If $\mathcal{F}$ is an exact category and $\Phi: \mathcal{E} \rightarrow \mathcal{F}$ is a left exact functor, then there exists a functor $\bar{\Phi}: \bmod _{\mathrm{adm}}(\mathcal{E}) \rightarrow \mathcal{F}^{\prime}$, unique up to isomorphism, which is exact and satisfies $\bar{\Phi} \circ \Upsilon=\Phi$.

Proof. 1. This follows from Lemma 3.2

2. Uniqueness is clear since any exact functor $\bmod _{\mathrm{adm}}(\mathcal{E}) \rightarrow \mathcal{F}$ is uniquely determined (up to isomorphism) by its restriction to the representable functors. We prove existence. For each $F \in \bmod _{\text {adm }}(\mathcal{E})$ choose a projective presentation $\Upsilon(A) \stackrel{\Upsilon(f)}{\longrightarrow} \Upsilon(B) \rightarrow F \rightarrow 0$ with $f$ admissible (where if $F=\Upsilon(E)$ we simply choose $0 \rightarrow \Upsilon(E) \stackrel{1}{\rightarrow} \Upsilon(E) \rightarrow 0$ ). Since $\Phi$ is left exact, it preserves admissible morphisms, and hence $\Phi(f)$ is admissible in $\mathcal{F}$. We define $\bar{\Phi}(F)=\operatorname{coker}(\Phi(f))$. Let $\eta: F \rightarrow F^{\prime}$ be a morphism in $\bmod _{\text {adm }}(\mathcal{E})$ between objects with fixed projective presentations $\Upsilon(A) \stackrel{\Upsilon(f)}{\longrightarrow} \Upsilon(B) \rightarrow F \rightarrow 0$ and $\Upsilon\left(A^{\prime}\right) \stackrel{\Upsilon\left(f^{\prime}\right)}{\longrightarrow} \Upsilon\left(B^{\prime}\right) \rightarrow F^{\prime} \rightarrow 0$. Choose maps $\alpha: A \rightarrow A^{\prime}$ and $\beta: B \rightarrow B^{\prime}$ in $\mathcal{E}$ making the diagram




commutative. We define $\bar{\Phi}(\eta): \bar{\Phi}(F) \rightarrow \bar{\Phi}\left(F^{\prime}\right)$ to be the unique morphism in $\mathcal{F}$ making the diagram

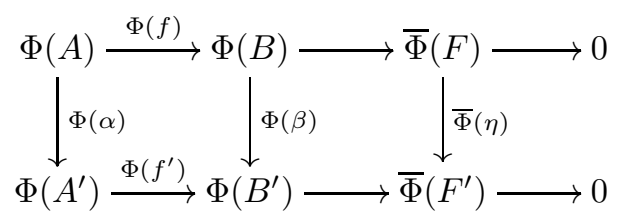

commutative. It is straightforward to check that $\bar{\Phi}(\eta)$ is independent of choice of $\alpha$ and $\beta$, and that $\bar{\Phi}: \bmod _{\mathrm{adm}}(\mathcal{E}) \rightarrow \mathcal{F}$ is a well-defined functor. It only remains to show that $\bar{\Phi}$ is exact. Let $F \longmapsto G \rightarrow H$ be a conflation in $\bmod _{\text {adm }}(\mathcal{E})$. Choose projective presentations $\Upsilon(A) \stackrel{\Upsilon(f)}{\longrightarrow} \Upsilon(B) \rightarrow$ $F \rightarrow 0$ and $\Upsilon(C) \stackrel{\Upsilon(h)}{\longrightarrow} \Upsilon(D) \rightarrow H \rightarrow 0$ of $F$ and $H$, and use the Horseshoe Lemma as in Proposition 3.4 to obtain the following commutative diagram in $\mathcal{E}$ :

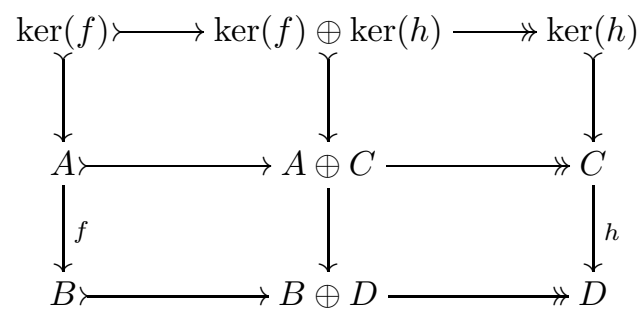

where the rows are are split kernel-cokernels pairs and the columns are left exact sequences. Since $\Phi$ is left exact and preserves split kernel-cokernel pairs, by applying the $3 \times 3$ lemma twice [24, Corollary 3.6] we get that $\bar{\Phi}(F) \longmapsto \bar{\Phi}(G) \rightarrow \bar{\Phi}(H)$ is a conflation. This shows that $\bar{\Phi}$ is exact.

Corollary 3.8. The Yoneda embedding $\Upsilon: \mathcal{E} \rightarrow \bmod _{a d m}(\mathcal{E})$ has an exact left adjoint $L: \bmod _{\text {adm }}(\mathcal{E}) \rightarrow \mathcal{E}$ satisfying $L \circ \Upsilon=1_{\mathcal{E}}$ and $\operatorname{ker}(L)=\operatorname{eff}(\mathcal{E})$.

Proof. Applying Theorem 3.7 to the identity functor on $\mathcal{E}$ gives an exact functor $L: \bmod _{\text {adm }}(\mathcal{E}) \rightarrow \mathcal{E}$ satisfying $L \circ \Upsilon=1_{\mathcal{E}}$. Explicitly, given $F \in \bmod _{\text {adm }}(\mathcal{E})$ with projective presentation $\Upsilon(A) \stackrel{\Upsilon(f)}{\longrightarrow} \Upsilon(B) \rightarrow$ $F$ where $f$ admissible, we have $L(F) \cong \operatorname{coker}(f)$. Note that $\Upsilon(B) \rightarrow \Upsilon(\operatorname{coker}(f))$ factors as $\Upsilon(Y) \rightarrow$ $F \stackrel{\alpha}{\rightarrow} \Upsilon(\operatorname{coker}(f))$ for a unique map $\alpha$. One readily verifies that any map $F \rightarrow \Upsilon(Z)$ must factor through $\alpha$ in a unique way, and hence we have natural isomorphisms

$$
\operatorname{Hom}_{\bmod _{\text {adm }}(\mathcal{E})}(F, \Upsilon(Z)) \cong \operatorname{Hom}_{\mathcal{E}}(\operatorname{coker}(f), Z) \cong \operatorname{Hom}_{\mathcal{E}}(L(F), Z)
$$

which shows that $L$ is left adjoint to $\Upsilon$. Finally, note that $L(F) \cong 0$ if and only if $\operatorname{coker}(f) \cong 0$. As $f$ is admissible, $\operatorname{coker}(f) \cong 0$ implies that $f$ is a deflation. This shows that $\operatorname{ker}(L)=\operatorname{eff}(\mathcal{E})$.

We now prove Auslander's formula for exact categories.

Theorem 3.9 (Auslander's formula for exact categories). The functor $L: \bmod _{a d m}(\mathcal{E}) \rightarrow \mathcal{E}$ induces an equivalence

$$
\bmod _{\mathrm{adm}}(\mathcal{E}) / \operatorname{eff}(\mathcal{E}) \cong \mathcal{E}
$$

of exact categories.

Proof. Since $\operatorname{ker}(L)=\operatorname{eff}(\mathcal{E})$, it follows that $L$ induces an exact functor $\bmod _{\text {adm }}(\mathcal{E}) / \operatorname{eff}(\mathcal{E}) \rightarrow \mathcal{E}$, which is an equivalence by [38, Proposition 1.3].

Corollary 3.10. 1. The Yoneda embedding $\Upsilon: \mathcal{E} \rightarrow \bmod _{\text {adm }}(\mathcal{E})$ lifts to a triangle equivalence $\mathrm{K}^{\mathrm{b}}(\mathcal{E}) \rightarrow$ $\mathrm{D}^{\mathrm{b}}\left(\bmod _{\mathrm{adm}}(\mathcal{E})\right)$ 
2. there is a natural commutative diagram

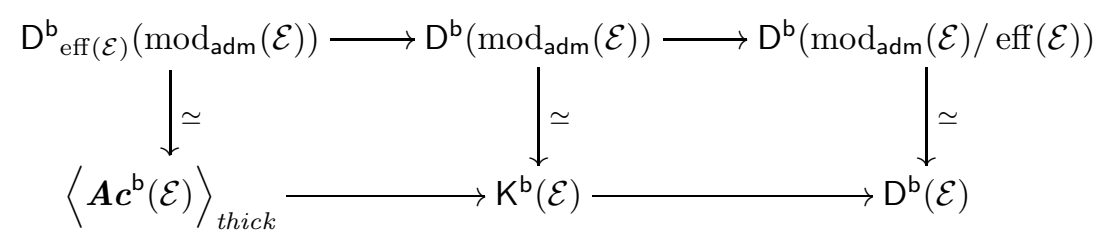

whose rows are Verdier localization sequences.

Proof. 1. This follows immediately since any object in $\bmod _{a d m}(\mathcal{E})$ has finite projective dimension by Lemma 3.2.

2. The upper Verdier localization sequence is induced by the localization sequence

$$
\operatorname{eff}(\mathcal{E}) \rightarrow \bmod _{\mathrm{adm}}(\mathcal{E}) \rightarrow \bmod _{\mathrm{adm}}(\mathcal{E}) / \operatorname{eff}(\mathcal{E}) .
$$

The lower sequence is simply the definition of $D^{\mathrm{b}}(\mathcal{E})$ for an exact category. The functor $L^{\prime}$ induces the equivalence $\mathrm{D}^{\mathrm{b}}\left(\bmod _{\mathrm{adm}}(\mathcal{E}) / \operatorname{eff}(\mathcal{E})\right) \stackrel{\simeq}{\rightarrow} \mathrm{D}^{\mathrm{b}}(\mathcal{E})$ and the Yoneda embedding induces the equivalence $\mathrm{K}^{\mathrm{b}}(\mathcal{E}) \rightarrow \mathrm{D}^{\mathrm{b}}\left(\bmod _{\mathrm{adm}}(\mathcal{E})\right)$. The result follows.

In the last part of this section we show that $\mathcal{E} \mapsto \bmod _{\text {adm }}(\mathcal{E})$ can be considered as a 2 -functor.

Definition 3.11. 1. Let Ex denote the 2-category with objects exact categories, with 1-morphisms exact functors, and with 2-morphisms natural transformations;

2. Let $\mathbf{E} \mathbf{x}_{L}$ denote the 2-category with objects exact categories, with 1-morphisms left exact functors, and with 2-morphisms natural transformations.

Corollary 3.12. The association $\mathcal{E} \mapsto \bmod _{\text {adm }}(\mathcal{E})$ induces a 2 -functor

$$
\bmod _{\mathrm{adm}}(-): \mathbf{E x}_{L} \rightarrow \mathbf{E x}
$$

which is left adjoint to the inclusion $\mathbf{E x} \subseteq \mathbf{E x}_{L}$, i.e. for which the restriction

$$
\operatorname{Hom}_{\mathbf{E x}}\left(\bmod _{\mathrm{adm}}(\mathcal{E}), \mathcal{E}^{\prime}\right) \rightarrow \operatorname{Hom}_{\mathbf{E x}_{L}}\left(\mathcal{E}, \mathcal{E}^{\prime}\right)
$$

via $\Upsilon: \mathcal{E} \rightarrow \bmod _{\mathrm{adm}}(\mathcal{E})$ is an equivalence of categories.

Proof. Using Theorem 3.7, for each left exact functor $\Phi: \mathcal{E} \rightarrow \mathcal{E}^{\prime}$ we choose an exact functor

$$
\bmod _{\mathrm{adm}}(\Phi): \bmod _{\mathrm{adm}}(\mathcal{E}) \rightarrow \bmod _{\mathrm{adm}}\left(\mathcal{E}^{\prime}\right)
$$

making the square

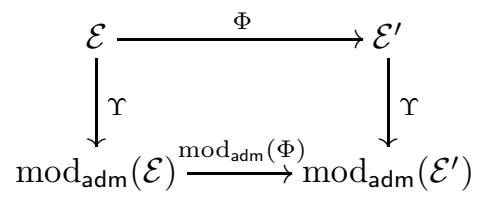

commutative. Since each natural transformation $\Phi \rightarrow \Phi^{\prime}$ can be extended uniquely to a natural transformation $\bmod _{\mathrm{adm}}(\Phi) \rightarrow \bmod _{\mathrm{adm}}\left(\Phi^{\prime}\right)$, we get a 2-functor $\bmod _{\mathrm{adm}}(-): \mathbf{E x}_{L} \rightarrow \mathbf{E x}$. Finally, the restriction $\operatorname{Hom}_{\mathrm{Ex}}\left(\bmod _{\mathrm{adm}}(\mathcal{E}), \mathcal{E}^{\prime}\right) \rightarrow \operatorname{Hom}_{\mathrm{Ex}_{L}}\left(\mathcal{E}, \mathcal{E}^{\prime}\right)$ is an equivalence by Theorem 3.7 and the fact that any natural transformation $\Psi \circ \Upsilon \rightarrow \Psi^{\prime} \circ \Upsilon$ where $\Psi, \Psi^{\prime} \in \operatorname{Hom}_{\mathrm{Ex}}\left(\bmod _{\mathrm{adm}}(\mathcal{E}), \mathcal{F}\right)$ can be extended uniquely to a natural transformation $\Psi \rightarrow \Psi^{\prime}$. 


\subsection{The torsion pair $(\operatorname{eff}(\mathcal{E}), \operatorname{cogen}(\mathcal{Q}))$ in $\bmod _{\mathrm{adm}}(\mathcal{E})$}

Fix an exact category $\mathcal{E}$ and set $\mathcal{Q}=\operatorname{Proj}\left(\bmod _{\text {adm }}(\mathcal{E})\right)$. In this section, we show that $(\operatorname{eff}(\mathcal{E}), \mathcal{F})$ as in Proposition 3.5 satisfies several useful properties. In the next section, we show that these properties can be used to characterize the image of the 2-functor $\bmod _{\mathrm{adm}}(-): \mathbf{E x}_{L} \rightarrow \mathbf{E x}$, which leads to a generalization of Auslander correspondence.

Definition 3.13. Let $\mathcal{X} \subseteq \mathcal{E}$ be a subcategory and $n \geq 0$ an integer.

1. We write $\operatorname{gen}_{k}(\mathcal{X})$ for the full subcategory of $\mathcal{E}$ consisting of those objects $E$ such that there is an exact sequence $X_{k} \rightarrow \cdots \rightarrow X_{2} \rightarrow X_{1} \rightarrow E \rightarrow 0$ with $X_{i} \in \mathcal{X}$ for $1 \leq i \leq k$. If $k=1$ we write $\operatorname{gen}_{1}(\mathcal{X})=\operatorname{gen}(\mathcal{X})$

2. We write $\operatorname{cogen}_{k}(\mathcal{X})$ for the full subcategory of $\mathcal{E}$ consisting of those objects $E$ such that there is an exact sequence $0 \rightarrow E \rightarrow X_{1} \rightarrow X_{2} \rightarrow \cdots \rightarrow X_{k}$ with $X_{i} \in \mathcal{X}$ for $1 \leq i \leq k$. If $k=1$ we write $\operatorname{cogen}_{1}(\mathcal{X})=\operatorname{cogen}(\mathcal{X})$

In the following ${ }^{\perp} \mathcal{Q}$ and $\operatorname{cogen}(\mathcal{Q})$ are defined inside the exact category $\bmod _{\text {adm }}(\mathcal{E})$.

Proposition 3.14. Consider the torsion pair $(\mathrm{eff}(\mathcal{E}), \mathcal{F})$ in $\bmod _{\mathrm{adm}}(\mathcal{E})$ as in Proposition 3.5. The following hold:

1. $\operatorname{eff}(\mathcal{E})={ }^{\perp} \mathcal{Q}$;

2. $\mathcal{F}=\operatorname{cogen}(\mathcal{Q})$;

3. $\operatorname{Ext}_{\bmod _{\text {adm }}(\mathcal{E})}^{1}(\operatorname{eff}(\mathcal{E}), \mathcal{Q})=0$;

4. The Yoneda functor $\Upsilon: \mathcal{E} \rightarrow \bmod _{\mathrm{adm}}(\mathcal{E})$ gives an equivalence $\mathcal{E} \cong \mathcal{Q}$.

Proof. $\quad$ 1. Clearly $\mathcal{Q} \subseteq \mathcal{F}$, hence the inclusion $\operatorname{eff}(\mathcal{E}) \subseteq{ }^{\perp} \mathcal{Q}$ follows from $\operatorname{Hom}_{\text {mod }_{\text {adm }}(\mathcal{E})}(\operatorname{eff}(\mathcal{E}), \mathcal{F})=0$. Conversely, let $G \in{ }^{\perp} \mathcal{Q}$. Choose a projective presentation $\Upsilon(A) \stackrel{\Upsilon(f)}{\longrightarrow} \Upsilon(B) \rightarrow G \rightarrow 0$ with $f$ admissible in $\mathcal{E}$. Since the composite $\Upsilon(A) \stackrel{\Upsilon(f)}{\longrightarrow} \Upsilon(B) \rightarrow \Upsilon(\operatorname{coker}(f))$ is 0 , the map $\Upsilon(B) \rightarrow$ $\Upsilon(\operatorname{coker}(f))$ factors through $G$ and must therefore be 0 since $G \in{ }^{\perp} \mathcal{Q}$. Using that $\Upsilon$ is faithful, it follows that the map $B \rightarrow \operatorname{coker}(f)$ is 0 , so $f$ must be a deflation. This shows that $G \in \operatorname{eff}(\mathcal{E})$.

2. We first show $\mathcal{F} \subseteq \operatorname{cogen}(\mathcal{Q})$. Choose $F \in \mathcal{F}$ and let $\Upsilon(X) \stackrel{\Upsilon(f)}{\longrightarrow} \Upsilon(Y) \rightarrow F \rightarrow 0$ be a projective presentation with $f$ an inflation. Let $g: Y \rightarrow Z$ denote the cokernel of $f$. By considering the two rightmost squares in diagram (1) in Proposition 3.3, we get that the commutative diagram

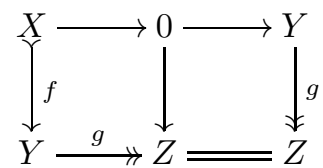

yields a short exact sequence $F \longmapsto \Upsilon(Z) \rightarrow H$ where $H=\operatorname{coker}(\Upsilon(g)) \in \operatorname{eff}(\mathcal{E})$. Thus $F \in \operatorname{cogen}(\mathcal{Q})$. Conversely, let $G \in \operatorname{cogen}(\mathcal{Q})$, then there is an inflation $G \longmapsto \Upsilon(Z)$ by definition. As $(\operatorname{eff}(\mathcal{E}), \mathcal{F})$ is a torsion pair, $G$ fits into a conflation $F \longmapsto G \rightarrow H$ with $F \in \operatorname{eff}(\mathcal{E})$ and $H \in \mathcal{F}$. As $\Upsilon(Z) \in \mathcal{F}$, the composition $F \longmapsto G \longmapsto \Upsilon(Z)$ is zero. It follows that $F \cong 0$ and thus $G \cong H \in \mathcal{F}$. This shows the equality $\mathcal{F}=\operatorname{cogen}(\mathcal{Q})$.

3. Let $E \in \operatorname{eff}(\mathcal{E})$. Then there exists a conflation $\operatorname{ker}(e) \stackrel{k}{\longmapsto} A \stackrel{e}{\rightarrow} B$ in $\mathcal{E}$ which gives a projective resolution

$$
0 \rightarrow \Upsilon(\operatorname{ker}(e)) \stackrel{\Upsilon(k)}{\longrightarrow} \Upsilon(A) \stackrel{\Upsilon(e)}{\longrightarrow} \Upsilon(B) \rightarrow E \rightarrow 0
$$

of $E$. Let $\Upsilon(Z) \in \mathcal{Q}$ be arbitrary. Applying the functor $\operatorname{Hom}(-, \Upsilon(Z))$ to the projective resolution and using that the $\Upsilon$ is fully faithful yields the left exact sequence

$$
0 \rightarrow \operatorname{Hom}(B, Z) \stackrel{-\circ e}{\longrightarrow} \operatorname{Hom}(A, Z) \stackrel{-\circ k}{\longrightarrow} \operatorname{Hom}(\operatorname{ker}(e), Z)
$$

of abelian groups. Since the sequence is exact at $\operatorname{Hom}(A, Z)$, it follows that $\operatorname{Ext}^{1}(E, \Upsilon(Z))=0$. 
4. Let $P \in \mathcal{Q}$. Note that $P$ is torsion-free and thus there exists a conflation $A \stackrel{f}{\longmapsto} B \rightarrow C$ such that $\Upsilon(A) \stackrel{\Upsilon(f)}{\longrightarrow} \Upsilon(B) \rightarrow P$ is a short exact sequence. Since $P$ is projective, the latter sequence is split and thus $f$ is a split inflation as $\Upsilon$ is fully faithful. It follows that $P \cong \Upsilon(C)$, which proves the claim.

Corollary 3.15. Let $\mathcal{E}$ be an exact category. The following hold:

1. The category $\mathcal{E}$ is weakly idempotent complete if and only if $\bmod _{\mathrm{adm}}(\mathcal{E})$ is weakly idempotent complete.

2. The category $\mathcal{E}$ is idempotent complete if and only if $\bmod _{\mathrm{adm}}(\mathcal{E})$ is idempotent complete.

Proof. The if direction follows as $\mathcal{E}$ is equivalent to $\operatorname{Proj}\left(\bmod _{\text {adm }}(\mathcal{E})\right)$. Conversely, assume that $\mathcal{E}$ is (weakly) idempotent complete, and let $f$ be an idempotent endomorphism (respectively a map which admits a right inverse) in $\bmod _{\mathrm{adm}}(\mathcal{E})$. Then $Q(f)$ is idempotent (respectively admits a right inverse), and hence $Q(f)$ is admissible by assumption. Therefore, by Theorem 2.10 we have that $f$ is admissible in $\bmod _{\mathrm{adm}}(\mathcal{E})$. Hence $\bmod _{\mathrm{adm}}(\mathcal{E})$ is (weakly) idempotent complete, which proves the claim.

\subsection{Admissibly presented functors and the category of sheaves}

Throughout this section, $\mathcal{E}$ denotes an exact category. The category $\bmod _{\text {adm }}(\mathcal{E})$ is defined as a subcategory of $\bmod (\mathcal{E})$, depending on the exact structure. In this section, we show how the category $\bmod _{\text {adm }}(\mathcal{E})$ occurs naturally by comparing the localization sequence eff $(\mathcal{E}) \rightarrow \bmod _{\text {adm }}(\mathcal{E}) \rightarrow \bmod _{\text {adm }}(\mathcal{E}) / \operatorname{eff}(\mathcal{E})$ from Theorem 3.9 to similar localization sequences appearing in the literature (see Proposition 3.23). In order to do so, we first recall the notion of a weakly effaceable functor as introduced by Grothendieck in [40] (following the terminology of [47]).

Definition 3.16. A functor $F \in \operatorname{Mod}(\mathcal{E})$ is called weakly effaceable if for every $C \in \mathcal{E}$ and every $x \in F(C)$, there exists a deflation $\lambda: C^{\prime} \rightarrow C$ such that $F(\lambda)(x)=0$. The full subcategory of $\operatorname{Mod}(\mathcal{E})$ of weakly effaceable functors is denoted by $\operatorname{Eff}(\mathcal{E})$.

The following proposition states that effaceable functors are weakly effaceable functors which are finitely presented.

Proposition 3.17. Let $F \in \operatorname{Mod}(\mathcal{E})$, then $F \in \operatorname{eff}(\mathcal{E})$ if and only if $F \in \operatorname{Eff}(\mathcal{E}) \cap \bmod (\mathcal{E})$.

Proof. Assume that $F \in \operatorname{eff}(\mathcal{E})$ and let $f: A \rightarrow B$ be a deflation in $\mathcal{E}$ such that $F \cong \operatorname{coker}(\Upsilon(f))$. For any $C \in \mathcal{E}$ and $x^{\prime} \in F(C)$, there exists a morphism $x: C \rightarrow B$ representing $x^{\prime}$. By axiom $\mathbf{R 2}$, we obtain the following pullback square:



Commutativity of the square yields $F(\lambda)(x)=0$. This shows that $F \in \operatorname{Eff}(\mathcal{E}) \cap \bmod (\mathcal{E})$.

Conversely, let $F \in \operatorname{Eff}(\mathcal{E}) \cap \bmod (\mathcal{E})$. As $F$ is finitely presented, there is a map $f: A \rightarrow B$ such that $F \cong \operatorname{coker}(\Upsilon(f))$. The identity $1_{B}$ yields an element $1 \in F(B)$. As $F \in \operatorname{Eff}(\mathcal{E})$, there exists a deflation $\lambda: C \rightarrow B$ such that $F(\lambda)(1)=0$. Hence there exists a map $u: C \rightarrow A$ such that $f u=\lambda 1_{B}=\lambda$. By $[44$, Proposition 3.4], the map $(0 f): C \oplus A \rightarrow B$ is a deflation in $\mathcal{E}$. Clearly, $\operatorname{coker}(\Upsilon((0 f))) \cong F$ and thus $F \in \operatorname{eff}(\mathcal{E})$.

Remark 3.18. It is shown in $[35$, Lemma 2.13] that $\operatorname{eff}(\mathcal{E}) \subseteq \operatorname{coh}(\mathcal{E})$ where $\operatorname{coh}(\mathcal{E})$ are the coherent functors, and thus $\operatorname{eff}(\mathcal{E})=\operatorname{Eff}(\mathcal{E}) \cap \operatorname{coh}(\mathcal{E})$. In particular, $\operatorname{eff}(\mathcal{E}) \subseteq \operatorname{coh}(\mathcal{E})$ is a Serre subcategory and thus $\operatorname{eff}(\mathcal{E})$ is abelian.

Corollary 3.19. $\quad$ 1. The category $\operatorname{Eff}(\mathcal{E})$ is a localizing Serre subcategory of $\operatorname{Mod}(\mathcal{E})$.

2. The category $\operatorname{eff}(\mathcal{E})$ is an admissibly-deflation percolating subcategory of $\bmod (\mathcal{E})$. 
Proof. That $\operatorname{Eff}(\mathcal{E})$ is a localizing Serre subcategory of $\operatorname{Mod}(\mathcal{E})$ is shown in [48, Appendix A.2]. Hence, by Proposition 3.17, $\operatorname{eff}(\mathcal{E}) \subseteq \bmod (\mathcal{E})$ is a Serre subcategory. That $\operatorname{eff}(\mathcal{E}) \subseteq \bmod (\mathcal{E})$ satisfies axiom A2 follows from Proposition 3.3.

Remark 3.20. By proposition 2.7, $\Sigma_{\operatorname{eff}(\mathcal{E})} \subseteq \operatorname{Mor}(\bmod (\mathcal{E}))$ is a right multiplicative system. Following [43], the localization functor $Q: \bmod (\mathcal{E}) \rightarrow \bmod (\mathcal{E})\left[\Sigma_{\text {eff }(\mathcal{E})}^{-1}\right]$ endows $\bmod (\mathcal{E})\left[\Sigma_{\text {eff }(\mathcal{E})}\right]$ with a deflation-exact structure (that is, $\bmod (\mathcal{E})\left[\Sigma_{\text {eff }(\mathcal{E})}^{-1}\right]$ satisfies axioms R0,R1 and R2), moreover, $\bmod (\mathcal{E})\left[\Sigma_{\text {eff }(\mathcal{E})}^{-1}\right]$ satisfies the universal property of a quotient of deflation-exact categories. As such, $\bmod (\mathcal{E})\left[\Sigma_{\text {eff }(\mathcal{E})}^{-1}\right] \simeq \bmod (\mathcal{E}) / \operatorname{eff}(\mathcal{E})$.

We are now in a position the consider the following commutative diagram for an exact category $\mathcal{E}$ :

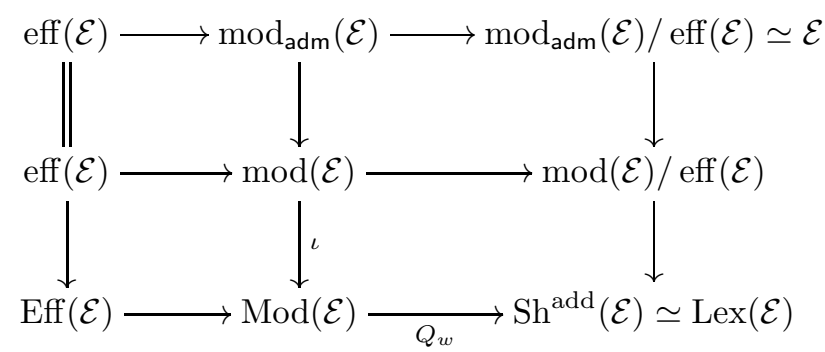

Here the bottom row is a Serre localization sequence of abelian categories as in [48, Appendix A.2] (see also [39, III.5.b]). The middle row is obtained by Remark 3.20 and the top row is Auslander's formula as in Theorem 3.9.

Remark 3.21. In [63], Rump considers a localization theory of left abelian categories. By [60, Proposition 5], left abelian categories with enough projectives are precisely categories of the form $\bmod (\mathcal{A})$ for an additive category $\mathcal{A}$. Any left abelian category has an exact structure given by all kernel-cokernel pairs [63, Proposition 2]. Rump defines a category $Q_{l}(\mathcal{E})$ which is obtained by localizing the left abelian category $\bmod (\mathcal{E})$ at a multiplicative system of morphisms, moveover, $Q_{l}(\mathcal{E})$ is exact (see [63, Theorem 1]).

There is a natural functor $\theta: \bmod (\mathcal{E}) / \operatorname{eff}(\mathcal{E}) \rightarrow Q_{l}(\mathcal{E})$ which is a localization functor by [42, Lemma 5.2]. By [63, Example 3], eff $(\mathcal{E})$ is a two-sided admissibly percolating subcategory if $\mathcal{E}$ is left quasi-abelian and then $\theta$ is an equivalence. Hence, the second row reduces to Rump's localization sequence in many cases. Furthermore, [63, corollary 2] shows that the embedding $\mathcal{E} \rightarrow \mathbf{Q}_{l}(\mathcal{E})$ is an equivalence if and only if $\mathcal{E}$ is abelian. In this case, the top and middle line of this diagram are equivalent.

We conclude this section by recovering $\bmod _{\mathrm{adm}}(\mathcal{E})$ directly from the bottom two localization sequences. We need the following lemma.

Lemma 3.22. Let $E \in \operatorname{eff}(\mathcal{E})$ and let $\Upsilon(\operatorname{ker}(g)) \longmapsto \Upsilon(X) \stackrel{\Upsilon(g)}{\longrightarrow} \Upsilon(Y) \rightarrow E$ be a resolution of $E$.

1. There exists an $X^{\prime} \in \mathcal{E}$ such that $(0 \mathrm{~g}): X^{\prime} \oplus X \rightarrow Y$ is a deflation.

2. If $\mathcal{E}$ is weakly idempotent complete, $g$ is a deflation.

Proof. As $E \in \operatorname{eff}(\mathcal{E})$, there is a resolution $\Upsilon(\operatorname{ker}(f)) \rightarrow \Upsilon(A) \stackrel{\Upsilon(f)}{\longrightarrow} \Upsilon(B) \rightarrow E$ with $f$ a deflation. By the Comparison Theorem, there is a homotopy equivalence between the two resolutions. As the Yoneda embedding is faithful, we obtain a homotopy equivalence between $\operatorname{ker}(g) \rightarrow X \stackrel{g}{\rightarrow} Y \rightarrow 0$ and $\operatorname{ker}(f) \rightarrow A \stackrel{f}{\rightarrow} B \rightarrow 0$. By [24, Proposition 10.14], $g$ is a deflation in the weak idempotent completion $\widehat{\mathcal{E}}$ of $\mathcal{E}$. This shows the first part, the second now follows from [44, Proposition 3.4].

Proposition 3.23. The category $\bmod _{\mathrm{adm}}(\mathcal{E})$ is obtained as the inverse image $\left(Q_{w} \circ \iota\right)^{-1}(\Upsilon(\mathcal{E}))$.

Proof. Let $F \in\left(Q_{w} \circ \iota\right)^{-1}(\Upsilon(\mathcal{E}))$. As $F \in \bmod (\mathcal{E})$, there is a map $f: A \rightarrow B$ such that $F \cong \operatorname{coker}(\Upsilon(f))$. As $Q_{w} \circ \iota(F) \in \Upsilon(\mathcal{E})$, there is a $C \in \mathcal{E}$ such that $Q_{w} \circ \iota(F) \cong \Upsilon(C)$. As $\operatorname{Eff}(\mathcal{E})$ is a localizing Serre subcategory of $\operatorname{Mod}(\mathcal{E})$ (see Corollary 3.19), the quotient functor $Q_{w}$ has a right adjoint $R$. The unit of the adjunction $Q_{w} \dashv R$ yields a map $u_{F}: \iota(F) \rightarrow R \Upsilon(C)$ such that $Q_{w}\left(u_{F}\right)$ is an isomorphism. It follows that $\operatorname{ker}\left(u_{F}\right), \operatorname{coker}\left(u_{F}\right) \in \operatorname{Eff}(\mathcal{E})$. 
Consider the following diagram

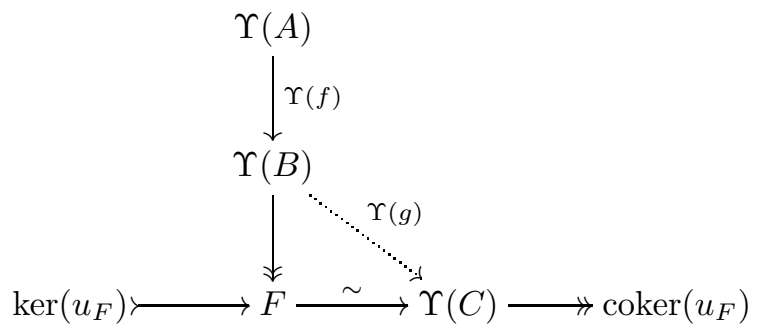

As the Yoneda functor is fully faithful, there is a map $g: B \rightarrow C$ such that the above diagram commutes. Note that $\operatorname{coker}\left(u_{F}\right) \cong \operatorname{coker}(\Upsilon(g))$. By Lemma 3.22, there is an object $B^{\prime} \in \mathcal{E}$ such that $(0 g): B^{\prime} \oplus B \rightarrow$ $C$ is a deflation. Write $K$ for the kernel of $(0 g)$. Consider the commutative diagram:

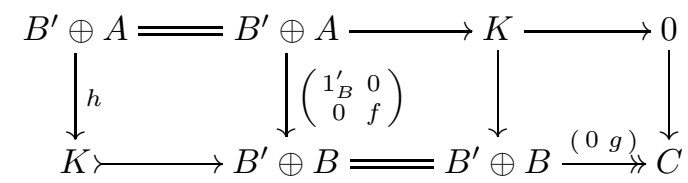

Using Proposition 3.3, the above diagram yields that $\operatorname{ker}\left(u_{F}\right) \cong \operatorname{coker}(\Upsilon(h))$. As $\operatorname{ker}\left(u_{F}\right) \in \operatorname{Eff}(\mathcal{E})$, Lemma 3.22 yields that $F \in \bmod _{\text {adm }}(\mathcal{E})$.

\section{Auslander correspondence for exact categories}

In this section we show that $\bmod _{\mathrm{adm}}(-): \mathbf{E x}_{L} \rightarrow \mathbf{E x}$ gives an equivalence onto a subcategory AEx of Ex, which we describe explicitly (see below). In particular, we obtain an extension of Auslander correspondence to exact categories.

\subsection{The 2-category AEx}

For an exact category $\mathcal{E}$ with enough projective objects, we let gl. $\operatorname{dim}(\mathcal{E})$ denote the global dimension of $\mathcal{E}$.

Definition 4.1. Let $\mathcal{E}$ be an exact category with enough projectives $\mathcal{P}=\operatorname{Proj}(\mathcal{E})$. We say that $\mathcal{E}$ is an Auslander exact category if it satisfies the following conditions:

(i) $\left({ }^{\perp} \mathcal{P}, \operatorname{cogen}(\mathcal{P})\right)$ is a torsion pair in $\mathcal{E}$;

(ii) ${ }^{\perp} \mathcal{P}$ satisfies axiom A2, i.e. all morphisms $f: E \rightarrow E^{\prime}$ with $E^{\prime} \in{ }^{\perp} \mathcal{P}$ are admissible with image in $\perp \mathcal{P}$;

(iii) $\operatorname{Ext}_{\mathcal{E}}^{1}\left({ }^{\perp} \mathcal{P}, \mathcal{P}\right)=0$;

(iv) $\operatorname{gl} \operatorname{dim}(\mathcal{E}) \leq 2$.

Definition 4.2. Let AEx denote the 2-category with objects Auslander exact categories, with 1-morphisms exact functors preserving projective objects, and with 2-morphisms natural transformations.

Corollary 4.3. $\bmod _{\mathrm{adm}}(-): \mathbf{E x}_{L} \rightarrow$ Ex factors through AEx.

Proof. By Lemma 3.2 we know that gl. $\operatorname{dim}\left(\bmod _{\mathrm{adm}}(\mathcal{E})\right) \leq 2$. The claim now follows from Proposition 3.5 and Proposition 3.14 


\subsection{The equivalence $E x_{L} \simeq \mathrm{AEx}$}

In this section we show that $\bmod _{\mathrm{adm}}(-): \mathbf{E x}_{L} \rightarrow \mathbf{A E x}$ is an equivalence of 2-categories.

Proposition 4.4. Let $\mathcal{E} \in \mathbf{A E x}$ and let $\mathcal{P}=\operatorname{Proj}(\mathcal{E})$. The following hold:

1. ${ }^{\perp} \mathcal{P} \subseteq \mathcal{E}$ is a two-sided admissibly percolating subcategory of $\mathcal{E}$;

2. Any weak ${ }^{\perp} \mathcal{P}$-isomorphism $P \stackrel{\sim}{\rightarrow} M$ with $P \in \mathcal{P}$ is a split inflation.

3. For every $M \in \operatorname{cogen}(\mathcal{P})$ there exists a conflation $M \stackrel{\sim}{\longmapsto} P \rightarrow T$ with $P \in \mathcal{P}$ and $T \in \perp \mathcal{P}$.

Proof. $\quad$ 1. This follows from Proposition 2.16.

2. If $f: P \stackrel{\sim}{\rightarrow} M$ be a weak ${ }^{\perp} \mathcal{P}$-isomorphism, then $f$ is admissible and $\operatorname{ker}(f), \operatorname{coker}(f) \in{ }^{\perp} \mathcal{P}$. Since $\operatorname{Hom}\left({ }^{\perp} \mathcal{P}, P\right)=0$, it follows that $\operatorname{ker}(f)=0$, and hence $f$ is an inflation. Since $\operatorname{Ext}_{\mathcal{E}}^{1}(\operatorname{coker}(f), P)=$ 0 , we get that $f$ must be a split inflation.

3. Let $M \in \operatorname{cogen}(\mathcal{P})$. By definition there is a conflation $M \longmapsto Q \rightarrow N$ with $Q \in \mathcal{P}$. Choose a conflation $\mathfrak{t} N \longmapsto N \rightarrow \mathfrak{f} N$ with $\mathfrak{t} N \in{ }^{\perp} \mathcal{P}$ and $\mathfrak{f} N \in \operatorname{cogen}(\mathcal{P})$. Taking the pullback of $Q \rightarrow N$ along $\mathfrak{t} N \longmapsto N$, we obtain the following commutative diagram:

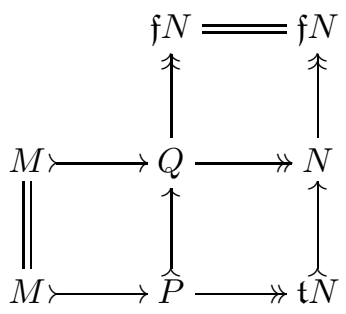

Since $\mathfrak{f} N \in \operatorname{cogen}(\mathcal{P})$ and $\operatorname{gl} \operatorname{dim}(\mathcal{E}) \leq 2$, it follows that $\mathfrak{f} N$ have projective dimension $\leq 1$. Thus $P$ must be projective. The lower row is therefore the desired conflation.

Proposition 4.5. Let $\mathcal{E} \in \mathbf{A E x}$ and let $\mathcal{P}=\operatorname{Proj}(\mathcal{E})$. The composition $\mathcal{P} \stackrel{\iota}{\hookrightarrow} \mathcal{E} \stackrel{Q}{\longrightarrow} \mathcal{E} / \perp^{\perp} \mathcal{P}$ is an equivalence of categories.

Proof. We first show that the composition $Q \circ \iota$ is essentially surjective. Let $M \in \mathrm{Ob}\left(\mathcal{E} /{ }^{\perp} \mathcal{P}\right)=\mathrm{Ob}(\mathcal{E})$. Choose a conflation $\mathfrak{t} M \longmapsto M \stackrel{\sim}{\rightarrow} \mathfrak{f} M$ with $\mathfrak{t} M \in{ }^{\perp} \mathcal{P}$ and $\mathfrak{f} M \in \operatorname{cogen}(\mathcal{P})$. By Proposition 4.4 . (3) there exists a conflation $\mathfrak{f} M \stackrel{\sim}{\longmapsto} P \rightarrow T$ with $P \in \mathcal{P}$ and $T \in{ }^{\perp} \mathcal{P}$. The composition $M \stackrel{\sim}{\rightarrow} \mathfrak{f} M \stackrel{\sim}{\longmapsto} P$ yield that $M \cong \mathfrak{f} M \cong P$ in $\Sigma_{\perp \mathcal{P}}^{-1} \mathcal{E}$. Hence $Q \circ \iota$ is essentially surjective.

We now show that $Q \circ \iota$ is faithful. Let $f: P_{1} \rightarrow P_{2}$ be a map in $\mathcal{P}$ such that $Q(f)=0$. As ${ }^{\perp} \mathcal{P}$ is a two-sided admissibly percolating subcategory of $\mathcal{E}$, the set $\Sigma_{\perp \mathcal{P}}$ is a left multiplicative system. It follows that there exists a weak isomorphism $P_{2} \stackrel{\sim}{\rightarrow} Y$ such that the composition $P_{1} \stackrel{f}{\rightarrow} P_{2} \stackrel{\sim}{\rightarrow} Y$ is zero. By Proposition 4.4.(2), $P_{2} \stackrel{\sim}{\rightarrow} Y$ is monic. It follows that $f$ is zero.

Lastly, we show that $Q \circ \iota$ is full. As $\Sigma_{\perp \mathcal{P}}$ is a left multiplicative system, we can represent a morphism $f \in \operatorname{Hom}_{\mathcal{E} / \perp^{\mathcal{P}}}\left(P_{1}, P_{2}\right)$ as a roof $P_{1} \longrightarrow X \stackrel{\sim}{\sim} P_{2}$. Then $P_{2} \stackrel{\sim}{\rightarrow} X$ has a left inverse $h: X \stackrel{\sim}{\rightarrow} P_{2}$ by Proposition 4.4.(2). The composite $P_{1} \rightarrow X \stackrel{h}{\rightarrow} P_{2}$ yields the desired morphism $g: P_{1} \rightarrow P_{2}$ such that $Q \circ \iota(g)=f$.

Proposition 4.6. Let $\mathcal{E} \in \mathbf{A E x}$ and let $\mathcal{P}=\operatorname{Proj}(\mathcal{E})$ be endowed with the exact structure coming from the equivalence in Proposition 4.5. The following hold:

1. The functor $\mathcal{P} \rightarrow \mathcal{E}$ is left exact;

2. The induced exact functor $\bmod _{\mathrm{adm}}(\mathcal{P}) \rightarrow \mathcal{E}$ is an equivalence. 
Proof. $\quad 1$. Let $P_{1} \stackrel{f}{\rightarrow} P_{2} \stackrel{g}{\rightarrow} P_{3}$ be a sequence in $\mathcal{P}$, and assume that $Q\left(P_{1}\right) \stackrel{Q(f)}{\longrightarrow} Q\left(P_{2}\right) \stackrel{Q(g)}{\longrightarrow} Q\left(P_{3}\right)$ is a conflation in $\mathcal{E} /{ }^{\perp} \mathcal{P}$. Since $Q(f)$ and $Q(g)$ are admissible in $\mathcal{E} /{ }^{\perp} \mathcal{P}$, it follows that $f$ and $g$ are admissible in $\mathcal{E}$ by Theorem 2.10. Hence, it only remains to show that $f$ is a kernel of $g$. Since $\mathcal{E}$ has enough projectives, it suffices to check that any morphism $h: P \rightarrow P_{2}$ with $P \in \mathcal{P}$ satisfying $g \circ h=0$ must factor uniquely through $f$. In other words, it suffices to show that the sequence

$$
\operatorname{Hom}_{\mathcal{E}}\left(P, P_{1}\right) \stackrel{f \circ-}{\longrightarrow} \operatorname{Hom}_{\mathcal{E}}\left(P, P_{2}\right) \stackrel{g \circ-}{\longrightarrow} \operatorname{Hom}_{\mathcal{E}}\left(P, P_{3}\right)
$$

is left exact. Now by Proposition 4.5 we have isomorphisms $\operatorname{Hom}_{\mathcal{E}}\left(P, P_{i}\right) \cong \operatorname{Hom}_{\mathcal{E} / \perp \mathcal{P}}\left(Q(P), Q\left(P_{i}\right)\right)$ for $1 \leq i \leq 3$. Hence the sequence above is isomorphic to the sequence

$$
\operatorname{Hom}_{\mathcal{E} /{ }^{\perp \mathcal{P}}}\left(Q(P), Q\left(P_{1}\right)\right) \stackrel{Q(f) \circ-}{\longrightarrow} \operatorname{Hom}_{\mathcal{E} /{ }^{\perp \mathcal{P}}}\left(Q(P), Q\left(P_{2}\right)\right) \stackrel{Q(g) \circ-}{\longrightarrow} \operatorname{Hom}_{\mathcal{E} /{ }^{\perp \mathcal{P}}}\left(Q(P), Q\left(P_{3}\right)\right)
$$

which is left exact since $Q\left(P_{1}\right) \stackrel{Q(f)}{\longrightarrow} Q\left(P_{2}\right) \stackrel{Q(g)}{\longrightarrow} Q\left(P_{3}\right)$ is a conflation. This proves the claim.

2. By Theorem 3.7 there exists an exact functor $\bmod _{\text {adm }}(\mathcal{P}) \rightarrow \mathcal{E}$ extending the inclusion $\mathcal{P} \rightarrow \mathcal{E}$. Since both $\bmod _{\text {adm }}(\mathcal{P})$ and $\mathcal{E}$ are exact categories with enough projectives, and since the exact functor $\bmod _{\text {adm }}(\mathcal{P}) \rightarrow \mathcal{E}$ restricts to an equivalence on the projective objects, it must be an equivalence itself.

Theorem 4.7 (First version of Auslander correspondence). $\bmod _{a d m}(-): \mathbf{E x}_{L} \rightarrow \mathbf{A E x}$ is an equivalence of 2-categories.

Proof. We define a 2-functor $\mathbf{A E x} \rightarrow \mathbf{E x}_{L}$ as follows: An object $\mathcal{E} \in \mathbf{A E x}$ is sent to $\operatorname{Proj}(\mathcal{E})$ endowed with the exact structure coming from the equivalence in Proposition 4.5. A 1-morphism $\Psi: \mathcal{E} \rightarrow \mathcal{E}^{\prime}$ is sent to the restriction $\left.\Psi\right|_{\operatorname{Proj}(\mathcal{E})}: \operatorname{Proj}(\mathcal{E}) \rightarrow \operatorname{Proj}\left(\mathcal{E}^{\prime}\right)$ (which can be seen to be left exact by using that the inclusion $\operatorname{Proj}(\mathcal{E}) \rightarrow \mathcal{E}$ is left exact). Finally, a natural transformation $\Psi \rightarrow \Psi^{\prime}$ is sent to the restriction $\left.\left.\Psi\right|_{\operatorname{Proj}(\mathcal{E})} \rightarrow \Psi^{\prime}\right|_{\operatorname{Proj}(\mathcal{E})}$. It follows from Theorem 3.9 and Proposition 4.6 that this gives a quasi-inverse to $\bmod _{\mathrm{adm}}(-)$, which proves the claim.

Remark 4.8. Note that $\mathcal{E}$ is an abelian category if and only if $\bmod _{\mathrm{adm}}(\mathcal{E})$ is an abelian category. Indeed, if $\mathcal{E}$ is abelian, then $\bmod _{\text {adm }}(\mathcal{E})=\bmod (\mathcal{E})$ is abelian since $\mathcal{E}$ has weak kernels [37]. Conversely, if $\bmod _{\mathrm{adm}}(\mathcal{E})$ is abelian then eff $(\mathcal{E})$ must be a Serre subcategory of $\bmod _{\mathrm{adm}}(\mathcal{E})$, and hence the localization $\mathcal{E} \cong \bmod _{\mathrm{adm}}(\mathcal{E}) / \mathrm{eff}(\mathcal{E})$ is an abelian category. It follows from this that $\bmod _{\text {adm }}(-): \mathbf{E x}_{L} \rightarrow \mathbf{A E x}$ restricts to an equivalence of 2 -categories

$$
\bmod (-): \mathbf{A} \mathbf{b}_{L} \rightarrow \mathbf{A A b}
$$

where $\mathbf{A} \mathbf{b}_{L}$ and $\mathbf{A A b}$ are the 2-subcategories of $\mathbf{E} \mathbf{x}_{L}$ and $\mathbf{A E x}$ consisting of the abelian categories. Note that axiom $\mathbf{A 2}$ in the definition of Auslander exact categories reduces to requiring ${ }^{\perp} \mathcal{P}$ to be closed under subobjects in this case. Note that Beligiannis obtained a similar characterization in [18, Theorem 6.6],

Using this equivalence, we deduce some further properties of Auslander exact categories.

Corollary 4.9. Let $\mathcal{E} \in \mathbf{A E x}$ and $\mathcal{P}=\operatorname{Proj}(\mathcal{E})$. The following hold:

1. The subcategory $\operatorname{cogen}(\mathcal{P})$ consists precisely of the objects of projective dimension at most one;

2. The inclusion functor $\mathcal{P} \rightarrow \mathcal{E}$ has a left adjoint.

Proof. This holds since the analogous properties are true for $\bmod _{\text {adm }}\left(\mathcal{E}^{\prime}\right)$ for an exact category $\mathcal{E}^{\prime}$ by Corollary 3.8 and Proposition 3.14. (2).

\subsection{Injectives and dominant dimension}

In this section we investigate how the property of an exact category $\mathcal{E}$ having enough injectives is reflected in $\bmod _{a d m}(\mathcal{E})$. In particular, we obtain a more familiar version of Auslander correspondence.

Lemma 4.10. Let $\mathcal{E}$ be an exact category. An object $I$ is injective in $\mathcal{E}$ if and only if $\Upsilon(I)$ is injective in $\bmod _{\mathrm{adm}} \mathcal{E}$. 
Proof. Assume $I$ is injective in $\mathcal{E}$. Let $F \in \bmod _{\text {adm }}(\mathcal{E})$, and choose an admissible morphism $f: X \rightarrow Y$ such that

$$
0 \rightarrow \Upsilon(\operatorname{ker} f) \rightarrow \Upsilon(X) \stackrel{\Upsilon(f)}{\longrightarrow} \Upsilon(Y) \rightarrow F \rightarrow 0
$$

is exact. Applying $\operatorname{Hom}_{\bmod _{a d m}(\mathcal{E})}(-, \Upsilon(I))$ and using that the Yoneda embedding is fully faithful, we get the sequence

$$
0 \rightarrow \operatorname{Hom}_{\bmod _{\text {adm }}(\mathcal{E})}(F, \Upsilon(I)) \rightarrow \operatorname{Hom}_{\mathcal{E}}(Y, I) \rightarrow \operatorname{Hom}_{\mathcal{E}}(X, I) \rightarrow \operatorname{Hom}_{\mathcal{E}}(\operatorname{ker} f, I) \rightarrow 0
$$

It is exact since $I$ is injective in $\mathcal{E}$. Hence $\operatorname{Ext}_{\text {modadm }_{(1)}(\mathcal{E})}(F, \Upsilon(I))=0$ for all $i>0$ and all $F \in \bmod _{\text {adm }}(\mathcal{E})$, so $\Upsilon(I)$ is injective in $\bmod _{\mathrm{adm}}(\mathcal{E})$.

Conversely, assume $\Upsilon(I)$ is injective in $\bmod _{\text {adm }}(\mathcal{E})$, and let $0 \rightarrow X \stackrel{f}{\rightarrow} Y \stackrel{g}{\rightarrow} Z \rightarrow 0$ be a conflation in $\mathcal{E}$. Then the sequence

$$
0 \rightarrow \Upsilon(X) \stackrel{\Upsilon(f)}{\longrightarrow} \Upsilon(Y) \stackrel{\Upsilon(g)}{\longrightarrow} \Upsilon(Z) \rightarrow \operatorname{coker}(\Upsilon(g)) \rightarrow 0
$$

is exact in $\bmod _{\mathrm{adm}}(\mathcal{E})$. Since $\operatorname{coker}(\Upsilon(g)) \in \operatorname{eff}(\mathcal{E})$, we get that $\operatorname{Hom}_{\bmod _{\text {adm }}(\mathcal{E})}(\operatorname{coker}(\Upsilon(g)), \Upsilon(I))=0$. Therefore, applying $\operatorname{Hom}_{\bmod _{\text {adm }}(\mathcal{E})}(-, \Upsilon(I))$ to the sequence above and using that

$$
\operatorname{Ext}_{\bmod _{\text {adm }}(\mathcal{E})}^{i}(\operatorname{coker}(\Upsilon(g)), \Upsilon(I))=0
$$

for all $i>0$ since $\Upsilon(I)$ is injective, we get an exact sequence

$$
0 \rightarrow \operatorname{Hom}_{\mathcal{E}}(Z, I) \stackrel{-\circ g}{\longrightarrow} \operatorname{Hom}_{\mathcal{E}}(Y, I) \stackrel{-\circ f}{\longrightarrow} \operatorname{Hom}_{\mathcal{E}}(X, I) \rightarrow 0 .
$$

This shows that $I$ is injective in $\mathcal{E}$.

Definition 4.11. Let $\mathcal{E}$ be an exact category with enough projectives. The dominant dimension of $\mathcal{E}$, denoted dom. $\operatorname{dim}(\mathcal{E})$, is the largest integer $n$ such that for any projective object $P \in \mathcal{E}$ there exists an exact sequence

$$
0 \rightarrow P \rightarrow I_{1} \rightarrow \cdots \rightarrow I_{n} \rightarrow C \rightarrow 0
$$

with $I_{k}$ being projective and injective for $1 \leq k \leq n$.

Lemma 4.12. Let $\mathcal{E}$ be an exact category. The following are equivalent:

1. $\mathcal{E}$ has enough injectives;

2. $\operatorname{dom} \cdot \operatorname{dim}\left(\bmod _{\mathrm{adm}}(\mathcal{E})\right) \geq 2$;

3. $\operatorname{dom} \cdot \operatorname{dim}\left(\bmod _{\mathrm{adm}}(\mathcal{E})\right) \geq 1$.

Proof. Assume $\mathcal{E}$ has enough injectives. By Proposition 3.14.(4), a projective object in $\bmod _{\mathrm{adm}}(\mathcal{E})$ is of the form $\Upsilon(X)$. As $\mathcal{E}$ has enough injectives, there is an exact sequence

$$
0 \rightarrow X \rightarrow I_{1} \stackrel{f}{\rightarrow} I_{2} \rightarrow \operatorname{coker}(f) \rightarrow 0
$$

with $I_{1}$ and $I_{2}$ injective. Applying the Yoneda embedding we get a left exact sequence

$$
0 \rightarrow \Upsilon(X) \rightarrow \Upsilon\left(I_{1}\right) \rightarrow \Upsilon\left(I_{2}\right)
$$

in $\bmod _{\mathrm{adm}}(\mathcal{E})$, and by Lemma 4.10 we know that $\Upsilon\left(I_{1}\right)$ and $\Upsilon\left(I_{2}\right)$ are projective and injective in $\bmod _{\mathrm{adm}}(\mathcal{E})$. This shows that dom. $\operatorname{dim}\left(\bmod _{\mathrm{adm}}(\mathcal{E})\right) \geq 2$. Since the implication $(2) \Rightarrow(3)$ is clear, it only remains to show $(3) \Rightarrow(1)$. Let $X$ be an arbitrary object in $\mathcal{E}$. By assumption there exists an inflation $\Upsilon(X) \rightarrow \Upsilon(I)$ where $\Upsilon(I)$ is injective in $\bmod _{\text {adm }}(\mathcal{E})$, and thus $I$ is injective in $\mathcal{E}$ by Lemma 4.10. Since the localization functor $Q: \bmod _{\mathrm{adm}}(\mathcal{E}) \rightarrow \bmod _{\mathrm{adm}}(\mathcal{E}) / \mathrm{eff}(\mathcal{E}) \simeq \mathcal{E}$ is exact, it follows that the induced morphism $X \rightarrow I$ is an inflation in $\mathcal{E}$. This proves the claim. PEx.

Our next goal is to show that $\operatorname{dom} \operatorname{dim}(\mathcal{E}) \geq 2$ implies some of the other criteria for categories in 
Lemma 4.13. Let $\mathcal{E}$ be an exact category with enough projectives $\mathcal{P}=\operatorname{Proj}(\mathcal{E}) . A$ ssume $\operatorname{dom} . \operatorname{dim}(\mathcal{E}) \geq 2$. Then $\operatorname{Ext}_{\mathcal{E}}^{1}\left({ }^{\perp} \mathcal{P}, \mathcal{P}\right)=0$.

Proof. Let $X \in{ }^{\perp} \mathcal{P}$ and $P \in \mathcal{P}$ be arbitrary. Choose a conflation $0 \rightarrow P \rightarrow I_{0} \rightarrow C \rightarrow 0$ and $0 \rightarrow C \rightarrow I_{1} \rightarrow C^{\prime} \rightarrow 0$ with $I_{0}$ and $I_{1}$ projective and injective in $\mathcal{E}$. Applying $\operatorname{Hom}_{\mathcal{E}}(X,-)$ to the second sequence, we get a monomorphism $\operatorname{Hom}_{\mathcal{E}}(X, C) \rightarrow \operatorname{Hom}_{\mathcal{E}}\left(X, I_{1}\right)$ and $\operatorname{since} \operatorname{Hom}_{\mathcal{E}}\left(X, I_{1}\right)=0$ by assumption on $X$, it follows that $\operatorname{Hom}_{\mathcal{E}}(X, C)=0$. Applying $\operatorname{Hom}_{\mathcal{E}}(X,-)$ to the first sequence we get an exact sequence $\operatorname{Hom}_{\mathcal{E}}(X, C) \rightarrow \operatorname{Ext}_{\mathcal{E}}^{1}(X, P) \rightarrow \operatorname{Ext}_{\mathcal{E}}^{1}(X, I)$. Now $\operatorname{Hom}_{\mathcal{E}}(X, C)=0$ by the discussion above and $\operatorname{Ext}_{\mathcal{E}}^{1}(X, I)=0$ since $I$ is injective. Hence $\operatorname{Ext}_{\mathcal{E}}^{1}(X, P)=0$, which proves the claim.

Lemma 4.14. Let $\mathcal{E}$ be an exact category with enough projectives $\mathcal{P}=\operatorname{Proj}(\mathcal{E})$. Assume $\operatorname{dom}$. $\operatorname{dim}(\mathcal{E}) \geq 1$. Then ${ }^{\perp} \mathcal{P}$ is closed under admissible subobjects.

Proof. Let $E \longmapsto X$ be an inflation with $X \in{ }^{\perp} \mathcal{P}$. We want to show that $E \in{ }^{\perp} \mathcal{P}$. Since $\operatorname{dom}$. $\operatorname{dim}(\mathcal{E}) \geq 1$, it suffices to show that $\operatorname{Hom}_{\mathcal{E}}(E, P)=0$ for all $P$ which are projective and injective. But since $P$ is injective, the induced map $\operatorname{Hom}_{\mathcal{E}}(X, P) \rightarrow \operatorname{Hom}_{\mathcal{E}}(E, P)$ is an epimorphism. Since $\operatorname{Hom}_{\mathcal{E}}(X, P)=0$ by assumption, it follows that $\operatorname{Hom}_{\mathcal{E}}(E, P)=0$.

Definition 4.15. Let $\mathcal{Y}$ be a subcategory of an exact category $\mathcal{E}$. A morphism $f: X \rightarrow Y$ in $\mathcal{E}$ with $Y \in \mathcal{Y}$ is called an (admissible) left- $\mathcal{Y}$ approximation if $f$ is an admissible morphism and any morphism $X \rightarrow Y^{\prime}$ with $Y^{\prime} \in \mathcal{Y}$ factors through $f$. The subcategory $\mathcal{Y}$ is called (admissibly) covariantly finite if for all objects $X$ in $\mathcal{E}$ there exists an admissible left $\mathcal{Y}$-approximation $X \rightarrow Y$.

Lemma 4.16. Let $\mathcal{E}$ be an exact category with enough projectives $\mathcal{P}=\operatorname{Proj}(\mathcal{E})$. The following hold:

1. If $\mathcal{E}$ is an Auslander exact category, then $\mathcal{P}$ is admissibly covariantly finite in $\mathcal{E}$;

2. If $\mathcal{P}$ is admissibly covariantly finite in $\mathcal{E}$ and $\operatorname{dom}$. $\operatorname{dim}(\mathcal{E}) \geq 1$, then $\left({ }^{\perp} \mathcal{P}, \operatorname{cogen}(\mathcal{P})\right)$ is a torsion pair.

Proof. 1. For $E \in \mathcal{E}$, choose a conflation $\mathfrak{t} E \longmapsto \mathcal{E} \rightarrow \mathfrak{f} E$ with $\mathfrak{t} E \in{ }^{\perp} \mathcal{P}$ and $\mathfrak{f} E \in \operatorname{cogen}(\mathcal{P})$. By Proposition 4.4 . (3) we can choose a conflation $\mathfrak{f} E \longmapsto P \rightarrow T$ with $P \in \mathcal{P}$ and $T \in{ }^{\perp} \mathcal{P}$. Since $\operatorname{Hom}_{\mathcal{E}}(\mathfrak{t} E, \mathcal{P})=0$, any morphism $E \rightarrow Q$ with $Q \in \mathcal{P}$ must factor through $\mathfrak{f} E$. Also, since $\operatorname{Ext}_{\mathcal{E}}^{1}(T, \mathcal{P})=0$, any morphism $\mathfrak{f} E \rightarrow Q$ with $Q \in \mathcal{P}$ must factor through $P$. This shows that $E \rightarrow \mathfrak{f} E \longmapsto P$ is the required left $\mathcal{P}$-approximation.

2. Let $X$ be an arbitrary object in $\mathcal{E}$, and choose an admissible left $\mathcal{P}$-approximation $f: X \rightarrow P$. Consider the conflation $\operatorname{ker}(f) \longmapsto X \rightarrow \operatorname{im}(f)$. Let $I$ be a projective and injective object in $\mathcal{E}$. Applying $\operatorname{Hom}_{\mathcal{E}}(-, I)$ to the conflation above, we get a short exact sequence of abelian groups

$$
\operatorname{Hom}_{\mathcal{E}}(\operatorname{im}(f), I) \longmapsto \operatorname{Hom}_{\mathcal{E}}(X, I) \rightarrow \operatorname{Hom}_{\mathcal{E}}(\operatorname{ker}(f), I) .
$$

Since $f$ is a left $\mathcal{P}$-approximation and $I$ is projective, it follows that $\operatorname{Hom}_{\mathcal{E}}(\operatorname{im}(f), I) \longmapsto \operatorname{Hom}_{\mathcal{E}}(X, I)$ is surjective, so $\operatorname{Hom}_{\mathcal{E}}(\operatorname{ker}(f), I)=0$. Since $\operatorname{dom}$. $\operatorname{dim}(\mathcal{E}) \geq 1$, any projective object is a subobject of a projective and injective object. Hence $\operatorname{ker}(f) \in{ }^{\perp} \mathcal{P}$. Since $\operatorname{im}(f) \in \operatorname{cogen}(\mathcal{P})$, this proves the claim.

We finish this section by showing that if $\mathcal{E}$ has enough injectives, then $\bmod _{\mathrm{adm}}(\mathcal{E})$ has enough injectives. In the following we let $\mathcal{I}$ denote the subcategory of injective objects in $\mathcal{E}$.

Remark 4.17. For all $n \geq 1, \operatorname{cogen}_{n}(\mathcal{I}) \subseteq \operatorname{cogen}_{n-1}(\mathcal{I}) \subseteq \cdots \subseteq \operatorname{cogen}_{1}(\mathcal{I})$. If $\mathcal{E}$ has enough injectives, then $\mathcal{E}=\operatorname{cogen}_{1}(\mathcal{I})=\operatorname{cogen}_{2}(\mathcal{I})=\cdots$.

Lemma 4.18. Let $\mathcal{E}$ be an exact category, let $n \geq 1$ be an integer, and let $X \mapsto Y \rightarrow Z$ be a conflation in $\mathcal{E}$. The following hold:

1. If $X \in \operatorname{cogen}_{n}(\mathcal{I})$ and $Z \in \operatorname{cogen}_{n}(\mathcal{I})$, then $Y \in \operatorname{cogen}_{n}(\mathcal{I})$;

2. If $X \in \operatorname{cogen}_{n}(\mathcal{I})$ and $Y \in \operatorname{cogen}_{n-1}(\mathcal{I})$, then $Z \in \operatorname{cogen}_{n-1}(\mathcal{I})$. 
Proof. 1. Choose exact sequences $0 \rightarrow X \rightarrow I_{1}^{X} \rightarrow \cdots \rightarrow I_{n}^{X} \rightarrow C^{X} \rightarrow 0$ and $0 \rightarrow Z \rightarrow I_{1}^{Z} \rightarrow \cdots \rightarrow$ $I_{n}^{Z} \rightarrow C^{Z} \rightarrow 0$ with each $I_{i}^{X}, I_{j}^{Z}$ injective. By the Horseshoe Lemma, there is an exact sequence

$$
0 \rightarrow Y \rightarrow I_{1}^{X} \oplus I_{Z}^{1} \rightarrow \cdots \rightarrow I_{n}^{X} \oplus I_{n}^{Z} \rightarrow C^{X} \oplus C_{Z} \rightarrow 0 .
$$

This shows that $Y \in \operatorname{cogen}_{n}(\mathcal{I})$.

2. As $X \in \operatorname{cogen}_{n}(\mathcal{I})$ and $n \geq 1$, there is a conflation $X \stackrel{i}{\longmapsto} I \rightarrow C$ with $I \in \operatorname{Inj}(\mathcal{E})$ and $C \in$ $\operatorname{cogen}_{n-1}(\mathcal{I})$.. Taking the pushout of $i$ along $X \mapsto Y$ we obtain the commutative diagram

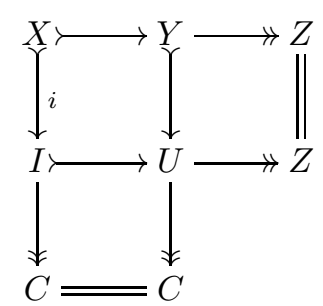

where the rows and columns are conflations. As $I$ is injective, the middle row is a split conflation and thus $U \cong I \oplus Z$. Applying the first part of this lemma to the conflation $Y \mapsto I \oplus Z \rightarrow C$ yields that $I \oplus Z \in \operatorname{cogen}_{n-1}(\mathcal{I})$. Hence there is an exact sequence

$$
0 \rightarrow I \oplus Z \rightarrow I_{1} \rightarrow \cdots \rightarrow I_{n-1} \rightarrow D \rightarrow 0
$$

with each $I_{j} \in \operatorname{Inj}(\mathcal{E})$. The composition $I \stackrel{\left(\begin{array}{l}1 \\ 0\end{array}\right)}{\longrightarrow} I \oplus Z \longmapsto I_{1}$ is a split inflation as $I$ is injective. It follows that $I_{1} \cong I \oplus I_{1}^{\prime}$ and $I_{1}^{\prime} \in \operatorname{Inj}(\mathcal{E})$ as injectives are closed under direct summands. The exact sequence above becomes

$$
0 \rightarrow I \oplus Z \stackrel{\left(\begin{array}{ll}
1 & 0 \\
0 & f
\end{array}\right)}{\longrightarrow} I \oplus I_{1}^{\prime} \rightarrow \cdots \rightarrow I_{n-1} \rightarrow D \rightarrow 0
$$

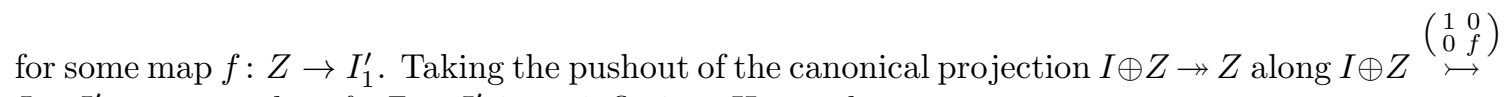
$I \oplus I_{1}^{\prime}$, one sees that $f: Z \longmapsto I_{1}^{\prime}$ is an inflation. Hence the sequence

$$
0 \rightarrow Z \stackrel{f}{\rightarrow} I_{1}^{\prime} \rightarrow I_{2} \rightarrow \cdots \rightarrow I_{n-1} \rightarrow D \rightarrow 0
$$

is exact, which shows that $Z \in \operatorname{cogen}_{n-1}(\mathcal{I})$.

Proposition 4.19. Let $\mathcal{E}$ be an exact category with enough projectives and assume

$$
\text { dom. } \operatorname{dim}(\mathcal{E}) \geq 2 \geq \text { gl. } \operatorname{dim}(\mathcal{E}) .
$$

Then $\mathcal{E}$ has enough injectives.

Proof. For any projective $P \in \mathcal{E}$, there exists an exact sequence

$$
0 \rightarrow P \rightarrow I_{1} \rightarrow I_{2} \rightarrow C \rightarrow 0
$$

where $I_{1}$ and $I_{2}$ are projective and injective. Since gl. $\operatorname{dim}(\mathcal{E}) \leq 2$ and $C$ is a cokernel of an admissible morphism between injective objects, it follows that $C$ is injective. Hence $P \in \operatorname{cogen}_{3}(\mathcal{I})$.

Now let $X \in \mathcal{E}$ be an arbitrary object, and choose an exact sequence $0 \rightarrow P_{2} \stackrel{f}{\rightarrow} P_{1} \rightarrow P_{0} \rightarrow X \rightarrow 0$ with $P_{0}, P_{1}, P_{2}$ projective in $\mathcal{E}$. Applying Lemma 4.18 to the conflation $P_{2} \stackrel{f}{\rightarrow} P_{1} \rightarrow \operatorname{coker}(f)$ and using that $P_{2}, P_{1} \in \operatorname{cogen}_{3}(\mathcal{I})$, it follows that $\operatorname{coker}(f) \in \operatorname{cogen}_{2}(\mathcal{I})$. Similarly, applying Lemma 4.18 to the conflation $\operatorname{coker}(f) \rightarrow P_{0} \rightarrow X$ we get that $X \in \operatorname{cogen}_{1}(\mathcal{I})$. This proves the claim.

Definition 4.20. Let $\mathbf{I E x _ { L }}$ be the 2-subcategory of $\mathbf{E x}_{L}$ consisting of the exact categories with enough injectives. 
Combining the results of this section, we obtain the following theorem.

Theorem 4.21 (Second version of Auslander correspondence). Let $\mathcal{E}$ be an exact category with enough projectives $\mathcal{P}=\operatorname{Proj}(\mathcal{E})$ and satisfying the following conditions:

(a) $\operatorname{dom} \cdot \operatorname{dim}(\mathcal{E}) \geq 2 \geq$ gl. $\operatorname{dim}(\mathcal{E})$;

(b) Any morphism $X \rightarrow E$ with $E \in{ }^{\perp} \mathcal{P}$ is admissible;

(c) $\mathcal{P}$ is admissibly covariantly finite.

The following hold:

1. $\mathcal{E}$ is an Auslander exact category with enough injectives;

2. The equivalence of 2-categories $\bmod _{\mathrm{adm}}(-): \mathbf{E x}_{L} \rightarrow \mathbf{A E x}$ restricts to an equivalence from $\mathbf{I E \mathbf { x } _ { L }}$ onto the subcategory of $\mathbf{A E x}$ consisting of the exact categories satisfying (a), (b) and (c) above.

Proof. 1. The category $\mathcal{E}$ has enough injectives by Proposition 4.19, and is an Auslander exact category by Lemma 4.13, Lemma 4.14 and Lemma 4.16. (2).

2. We only need to verify that $\mathcal{E}$ has enough injectives if and only if $\bmod _{\text {adm }}(\mathcal{E})$ satisfies (a), (b) and (c) above. The "only if" direction follows from Lemma 4.12, and the "if" direction follows from Lemma 4.12 and Lemma 4.16. (1).

Remark 4.22. Note that the abelian categories satisfying (a) in Theorem 4.21 are precisely the free abelian categories, see [18, Theorem 6.6]

Remark 4.23. We explain how to recover the classical Auslander correspondence. Let $k$ be a field. Assume $\Lambda$ is a representation-finite finite-dimensional algebra, and choose an additive generator $M$ of $\bmod \Lambda$. We then have an equivalences of categories

$$
\bmod \Gamma \cong \bmod (\bmod (\Lambda))
$$

where $\Gamma=\operatorname{End}_{\Lambda}(M)$. Since $\bmod (\Lambda)$ is an abelian category with enough injectives, we get that

$$
\text { dom. } \operatorname{dim} \Gamma \geq 2 \geq \text { gl. } \operatorname{dim} \Gamma
$$

by Theorem 4.21. This gives one direction of the classical Auslander correspondence.

Conversely, assume $\Gamma$ is a finite-dimensional algebra satisfying $\operatorname{dom}$. $\operatorname{dim} \Gamma \geq 2 \geq \mathrm{gl}$. $\operatorname{dim} \Gamma$. Since $\Gamma$ is finite-dimensional, the subcategory $\operatorname{proj} \Gamma$ of projective objects in $\bmod (\Gamma)$ is a covariantly finite subcategory. Hence, $\bmod (\Gamma)$ satisfies (a), (b) and (c) in Theorem 4.21, and combining this with Remark 4.8 we therefore get that $\operatorname{proj} \Gamma$ is an abelian category with enough injectives. Hence, $(\operatorname{proj} \Gamma)^{\mathrm{op}} \cong \bmod \left(\Lambda^{\prime}\right)$ for some finite-dimensional algebra $\Lambda^{\prime}$. Composing with the duality

$$
\operatorname{Hom}_{k}(-, k): \bmod \left(\Lambda^{\prime \mathrm{op}}\right) \stackrel{\cong}{\rightrightarrows}\left(\bmod \left(\Lambda^{\prime}\right)\right)^{\mathrm{op}}
$$

we get that $\operatorname{proj} \Gamma \cong \bmod \left(\Lambda^{\prime \text { op }}\right)$. This recovers the other direction of the classical Auslander correspondence.

\subsection{Projective objects}

In this section we investigate how the property of an exact category $\mathcal{E}$ having enough projectives is reflected in $\bmod _{\text {adm }}(\mathcal{E})$.

Lemma 4.24. An object $P$ of an exact category $\mathcal{E}$ is projective if and only if $\Upsilon(P) \in{ }^{\perp} \operatorname{eff}(\mathcal{E})$.

Proof. Let $F \in \bmod _{\text {adm }}(\mathcal{E})$, and consider the projective presentation

$$
\Upsilon(X) \stackrel{\Upsilon(f)}{\longrightarrow} \Upsilon(Y) \rightarrow F \rightarrow 0
$$


where $f: X \rightarrow Y$ is admissible. Applying $\operatorname{Hom}_{\text {modadm }_{(1)}(\mathcal{E})}(\Upsilon(P),-)$ gives an exact sequence

$$
\operatorname{Hom}_{\mathcal{E}}(P, X) \stackrel{f \circ-}{\longrightarrow} \operatorname{Hom}_{\mathcal{E}}(P, Y) \rightarrow \operatorname{Hom}_{\text {mod }_{\text {adm }}(\mathcal{E})}(\Upsilon(P), F) \rightarrow 0
$$

of abelian groups. Now by definition $P$ is projective if and only if for any deflation $f: X \rightarrow Y$ the induced map

$$
\operatorname{Hom}_{\mathcal{E}}(P, X) \stackrel{f \circ-}{\longrightarrow} \operatorname{Hom}_{\mathcal{E}}(P, Y)
$$

is an epimorphism. Hence, by the exact sequence above we get that $P$ is projective if and only $\operatorname{Hom}_{\bmod _{\text {adm }}(\mathcal{E})}(\Upsilon(P), F)=0$ for any $F \in \operatorname{eff}(\mathcal{E})$. This proves the claim.

Proposition 4.25. Let $\mathcal{E}$ be an exact category and write $\mathcal{Q}$ for $\operatorname{Proj}\left(\bmod _{a d m}(\mathcal{E})\right)$. The following are equivalent:

1. The category $\mathcal{E}$ has enough projectives;

2. $\left(\operatorname{gen}\left(\mathcal{Q} \cap{ }^{\perp} \operatorname{eff}(\mathcal{E})\right)\right.$, eff $\left.(\mathcal{E})\right)$ is a torsion pair in $\bmod _{\mathrm{adm}}(\mathcal{E})$;

3. For all $P \in \mathcal{Q}$, there exists an exact sequence

$$
0 \rightarrow F^{\prime} \rightarrow P \rightarrow F^{\prime \prime} \rightarrow 0
$$

with $F^{\prime} \in \operatorname{gen}\left(\mathcal{Q} \cap{ }^{\perp} \operatorname{eff}(\mathcal{E})\right)$ and $F^{\prime \prime} \in \operatorname{eff}(\mathcal{E})$.

Proof. Assume (1). We first show that $\operatorname{Hom}\left(\operatorname{gen}\left(\mathcal{Q} \cap{ }^{\perp} \operatorname{eff}(\mathcal{E})\right)\right.$, eff $\left.(\mathcal{E})\right)=0$. Let $g: E \rightarrow F$ be a map with $E \in \operatorname{gen}\left(\mathcal{Q} \cap{ }^{\perp} \operatorname{eff}(\mathcal{E})\right)$ and $F \in \operatorname{eff}(\mathcal{E})$. By definition, there is an object $E^{\prime} \in \mathcal{Q} \cap{ }^{\perp} \operatorname{eff}(\mathcal{E})$ and a deflation $E^{\prime} \rightarrow E$. By Proposition 3.14. (4) the functor $\Upsilon: \mathcal{E} \rightarrow \mathcal{Q}$ is an equivalence and thus there is an object $P \in \mathcal{E}$ such that $\Upsilon(P) \cong E^{\prime}$. By Lemma 4.24, $P \in \operatorname{Proj}(\mathcal{E})$. Let $f: X \rightarrow Y$ be a deflation in $\mathcal{E}$ such that $\operatorname{coker}(\Upsilon(f)) \cong F$. Since $\Upsilon(P)$ is projective and $\Upsilon$ is fully faithful, there exists a map $h: P \rightarrow Y$ such that the composite $\Upsilon(P) \rightarrow E \stackrel{g}{\rightarrow} F$ is equal to $\Upsilon(P) \stackrel{\Upsilon(h)}{\longrightarrow} \Upsilon(Y) \rightarrow F$. Now since $f$ is a deflation and $P$ is projective, $h$ must factor through $f$, and hence the composite above must be 0 . As $\Upsilon(P) \rightarrow E$ is surjective, $g$ must be zero. We conclude that $\operatorname{Hom}\left(\operatorname{gen}\left(\mathcal{Q} \cap{ }^{\perp} \operatorname{eff}(\mathcal{E})\right)\right.$, eff $\left.(\mathcal{E})\right)=0$.

Let $F \in \bmod _{\text {adm }}(\mathcal{E})$ and let $F \cong \operatorname{coker}(\Upsilon(f))$ for some admissible morphism $f: X \rightarrow Y$ in $\mathcal{E}$. As $\mathcal{E}$ has enough projectives, there is a deflation $P \stackrel{p}{\rightarrow} Y$ with $P \in \operatorname{Proj}(\mathcal{E})$. The pullback $Z$ of $f$ along $p$ yields an admissible morphism $g: Z \rightarrow P$. Note that this pullback square induces a conflation $Z \hookrightarrow X \oplus P \stackrel{(f p)}{\rightarrow} Y$ by (the dual of) [24, Proposition 2.12]. Let $F^{\prime}=\operatorname{coker}(\Upsilon(g))$ and let $F^{\prime \prime}=\operatorname{coker}(\Upsilon(f p)) \in \operatorname{eff}(\mathcal{E})$. Considering the two rightmost squares in diagram (1) in Proposition 3.3, we see that we have a conflation $F^{\prime} \longmapsto F \rightarrow F^{\prime \prime}$. It remains to show that $F^{\prime} \in \operatorname{gen}\left(\mathcal{Q} \cap{ }^{\perp} \operatorname{eff}(\mathcal{E})\right)$. By construction there is a deflation $\Upsilon(P) \rightarrow F^{\prime}$ and as $P \in \operatorname{Proj}(\mathcal{E})$, Lemma 4.24 yields that $\Upsilon(P) \in{ }^{\perp} \operatorname{eff}(\mathcal{E})$, thus $F^{\prime} \in \operatorname{gen}\left(\mathcal{Q} \cap{ }^{\perp} \operatorname{eff}(\mathcal{E})\right.$ ). This shows the implication $(1) \Rightarrow(2)$.

The implication $(2) \Rightarrow(3)$ is trivial. Now assume (3). Let $A \in \mathcal{E}$. By assumption there is a short exact sequence

$$
F^{\prime} \longmapsto \Upsilon(A) \rightarrow F^{\prime \prime}
$$

with $F^{\prime} \in \operatorname{gen}\left(\mathcal{Q} \cap{ }^{\perp} \operatorname{eff}(\mathcal{E})\right)$ and $F^{\prime \prime} \in \operatorname{eff}(\mathcal{E})$. By definition there is a deflation $\Upsilon(P) \rightarrow F^{\prime}$ with $P \in$ $\operatorname{Proj}(\mathcal{E})$. Letting $K$ be the kernel of the map $\Upsilon(P) \rightarrow F^{\prime} \longmapsto \Upsilon(A)$, we get an exact sequence

$$
0 \rightarrow K \rightarrow \Upsilon(P) \rightarrow \Upsilon(A) \rightarrow F^{\prime \prime} \rightarrow 0
$$

in $\bmod _{\mathrm{adm}}(\mathcal{E})$. Applying the functor $L: \bmod _{\mathrm{adm}}(\mathcal{E}) \rightarrow \mathcal{E}$ in Corollary 3.8 and using that $L\left(F^{\prime \prime}\right)=0$ since $F^{\prime \prime} \in \operatorname{eff}(\mathcal{E})$, we get the conflation $L(K) \longmapsto P \rightarrow A$ in $\mathcal{E}$. Hence $\mathcal{E}$ has enough projectives.

\subsection{Gorenstein projectives and Cohen-Macaulay modules}

If $\mathcal{E}^{\prime}$ is an extension closed subcategory of an exact category $\mathcal{E}$, then $\mathcal{E}^{\prime}$ inherits an exact structure from $\mathcal{E}$. In this case, since the inclusion $\mathcal{E}^{\prime} \rightarrow \mathcal{E}$ is fully faithful and exact, applying the 2 -functor $\bmod _{\text {adm }}(-)$ we get a fully faithful exact functor

$$
\bmod _{\mathrm{adm}}\left(\mathcal{E}^{\prime}\right) \rightarrow \bmod _{\mathrm{adm}}(\mathcal{E}) .
$$


Hence $\bmod _{\mathrm{adm}}\left(\mathcal{E}^{\prime}\right)$ is equivalent to a full subcategory of $\bmod _{\mathrm{adm}}(\mathcal{E})$. Our goal in this section is to characterize this subcategory in certain examples. In the following $Q: \bmod _{\text {adm }}(\mathcal{E}) \rightarrow \mathcal{E}$ denotes the localization functor, $\Upsilon\left(\mathcal{E}^{\prime}\right) \subseteq \bmod _{\text {adm }}(\mathcal{E})$ denotes the subcategory of objects of the form $\Upsilon(E)$ with $E \in \mathcal{E}^{\prime}$, and $Q^{-1}\left(\mathcal{E}^{\prime}\right) \subseteq \bmod _{\mathrm{adm}}(\mathcal{E})$ denotes the subcategory of objects $F$ satisfying $Q(F) \in \mathcal{E}^{\prime}$.

Lemma 4.26. Let $\mathcal{E}$ be an exact category, and let $\mathcal{E}^{\prime}$ be a subcategory of $\mathcal{E}$ closed under extensions and kernels of deflations. Then $\bmod _{\mathrm{adm}}\left(\mathcal{E}^{\prime}\right)$ is equivalent to the subcategory $Q^{-1}\left(\mathcal{E}^{\prime}\right) \cap \operatorname{gen}_{2}\left(\Upsilon\left(\mathcal{E}^{\prime}\right)\right)$ of $\bmod _{\mathrm{adm}}(\mathcal{E})$.

Proof. An object $F \in \bmod _{\mathrm{adm}}(\mathcal{E})$ is in the essential image of the functor $\bmod _{\mathrm{adm}}\left(\mathcal{E}^{\prime}\right) \rightarrow \bmod _{\mathrm{adm}}(\mathcal{E})$ if and only if there exists a projective presentation

$$
\Upsilon(X) \stackrel{\Upsilon(f)}{\longrightarrow} \Upsilon(Y) \rightarrow F \rightarrow 0
$$

where $X, Y \in \mathcal{E}^{\prime}$ and $f$ is admissible in $\mathcal{E}^{\prime}$. Since $Q(F)=\operatorname{coker}(f)$, it is clear that the essential image is contained in $Q^{-1}\left(\mathcal{E}^{\prime}\right) \cap \operatorname{gen}_{2}\left(\Upsilon\left(\mathcal{E}^{\prime}\right)\right)$. Conversely, if $F$ is in $Q^{-1}\left(\mathcal{E}^{\prime}\right) \cap \operatorname{gen}_{2}\left(\Upsilon\left(\mathcal{E}^{\prime}\right)\right)$, then there exists a projective presentation (2) where $X, Y \in \mathcal{E}^{\prime}$. Since $Q$ preserves admissible morphisms, it follows that $f$ is admissible and $\operatorname{coker}(f) \in \mathcal{E}^{\prime}$. But then $f$ must be admissible in $\mathcal{E}^{\prime}$, since $\mathcal{E}^{\prime}$ is closed under kernels of deflations. This proves the claim.

We get the following corollary for subcategories of abelian categories with enough projectives.

Proposition 4.27. Let $\mathcal{P}$ be an additive category, and assume $\bmod (\mathcal{P})$ is an abelian category. Let $\mathcal{X}$ be a subcategory of $\bmod (\mathcal{P})$ closed under extensions, kernels of deflations, and which contain $\Upsilon(\mathcal{P})$. Then

$$
\bmod _{\mathrm{adm}}(\mathcal{X})=\left\{F \in \bmod (\mathcal{X})|F|_{\mathcal{P}} \in \mathcal{X}\right\}
$$

Proof. Note that the localization $Q: \bmod (\bmod (\mathcal{P})) \rightarrow \bmod (\mathcal{P})$ is given by the restriction functor $Q(F)=$ $\left.F\right|_{\mathcal{P}}$. The claim follows therefore from Lemma 4.26 and the fact that $\bmod (\mathcal{X})$ can be identified with the subcategory $\operatorname{gen}_{2}(\Upsilon(\mathcal{X}))$ of $\bmod (\mathcal{A})$.

In particular, Proposition 4.27 holds in the following cases:

- $\mathcal{X}$ is the subcategory $\operatorname{GP}(\mathcal{P})$ of Gorenstein-projective objects in $\bmod (\mathcal{P})$.

- $\mathcal{X}$ is the subcategory of objects of projective dimension $\leq i$ for some integer $i$.

- $\mathcal{X}$ is the subcategory of objects of finite projective dimension.

- $\mathcal{X}=>{ }^{\perp} U=\left\{M \in \bmod (\mathcal{P}) \mid \operatorname{Ext}_{\bmod (\mathcal{P})}^{i}(M, U)=0\right.$ for all $\left.i>0\right\}$ for some object $U \in \bmod (\mathcal{P})$. In particular, this covers orthogonal categories of cotilting modules as studied in [45] and [34].

- $R$ is a commutative Cohen-Macaulay local ring, $\Lambda$ is an $R$-order, i.e. a noetherian $R$-algebra which is maximal Cohen-Macaulay as an $R$-module, and $\mathcal{X}$ is the subcategory

$$
\operatorname{CM}(\Lambda)=\{M \in \bmod (\Lambda) \mid M \text { is maximal Cohen-Macaulay as an } R \text {-module }\}
$$

of maximal Cohen-Macaulay modules of $\Lambda$.

We end this section by restating Proposition 4.27 when the subcategories of Gorenstein projective modules or Cohen-Macaulay modules are of finite type. Here, for an exact category $\mathcal{E}^{\prime}$ with an additive generator $M$ and endomorphism $\operatorname{ring} \Gamma=\operatorname{End}_{\mathcal{E}^{\prime}}(M)$, we let $\bmod _{\text {adm }}(\Gamma)$ be the subcategory of $\bmod (\Gamma)$ which corresponds to the subcategory $\bmod _{\mathrm{adm}}\left(\mathcal{E}^{\prime}\right)$ under the equivalence $\bmod (\Gamma) \cong \bmod \left(\mathcal{E}^{\prime}\right)$.

Corollary 4.28. Let $\Lambda$ be a noetherian ring, and assume the subcategory $\operatorname{GP}(\Lambda)$ of Gorenstein projective modules in $\bmod (\Lambda)$ has an additive generator $M \in \operatorname{GP}(\Lambda)$. Assume also for simplicity that $\Lambda$ is a summand of $M$. Set $\Gamma=\operatorname{End}_{\Lambda}(M)$ and let $e \in \Gamma$ be the idempotent corresponding to $\Lambda$, so that $e \Gamma e \cong \Lambda$. Then

$$
\bmod _{\mathrm{adm}}(\Gamma)=\{M \in \bmod (\Gamma) \mid M e \in \mathrm{GP}(\Lambda)\} .
$$

Corollary 4.29. Let $\Lambda$ be an $R$-order over a local commutative Cohen-Macaulay ring $R$, and assume the subcategory $\mathrm{CM}(\Lambda)$ of maximal Cohen-Macaulay has an additive generator $M \in \mathrm{CM}(\Lambda)$. Assume also for simplicity that $\Lambda$ is a summand of $M$. Set $\Gamma=\operatorname{End}_{\Lambda}(M)$ and let $e \in \Gamma$ be the idempotent corresponding to $\Lambda$. Then

$$
\bmod _{\mathrm{adm}}(\Gamma)=\{M \in \bmod (\Gamma) \mid M e \in \mathrm{CM}(\Lambda)\}
$$




\section{Characterizing exact structures via resolving subcategories}

Let $\mathcal{C}$ be an idempotent complete additive category. In this section we characterize the subcategories of $\bmod (\mathcal{C})$ of the form $\bmod _{\text {adm }}(\mathcal{E})$ where $\mathcal{E}$ is an exact category obtained by choosing an exact structure on $\mathcal{C}$.

\subsection{Resolving subcategories}

Fix an idempotent complete exact category $\mathcal{E}$. Our goal in this section is to investigate the relationship between $\operatorname{eff}(\mathcal{E})$ and $\bmod _{\mathrm{adm}}(\mathcal{E})$ as subcategories of $\bmod (\mathcal{E})$.

Definition 5.1. A subcategory $\mathcal{X}$ of $\mathcal{E}$ is called resolving if it is closed under extensions, direct summands, and kernel of deflations, and if it is generating, i.e. $\operatorname{gen}(\mathcal{X})=\mathcal{E}$.

Definition 5.2. Let $\mathcal{P}^{2}(\mathcal{E})$ denote the subcategory of $\bmod (\mathcal{E})$ consisting of all $F$ for which there exists an exact sequence

$$
0 \rightarrow \operatorname{Hom}_{\mathcal{E}}(-, X) \rightarrow \operatorname{Hom}_{\mathcal{E}}(-, Y) \rightarrow \operatorname{Hom}_{\mathcal{E}}(-, Z) \rightarrow F \rightarrow 0 .
$$

Lemma 5.3. $\mathcal{P}^{2}(\mathcal{E})$ is closed under extensions, direct summands and kernels of epimorphisms.

Proof. $\mathcal{P}^{2}(\mathcal{E})$ is the subcategory of objects of projective dimension $\leq 2$ in the category $\bmod _{\infty}(\mathcal{E})$ consisting of all $F$ which admits an exact sequence

$$
\cdots \rightarrow \operatorname{Hom}_{\mathcal{E}}\left(-, X_{2}\right) \rightarrow \operatorname{Hom}_{\mathcal{E}}\left(-, X_{1}\right) \rightarrow F \rightarrow 0 .
$$

Since $\bmod _{\infty}(\mathcal{E})$ is closed under extensions, direct summands and kernels of epimorphisms by [33, Proposition 2.6], the same must hold for $\mathcal{P}^{2}(\mathcal{E})$.

Proposition 5.4. $\bmod _{\mathrm{adm}}(\mathcal{E})$ is the smallest resolving subcategory of $\mathcal{P}^{2}(\mathcal{E})$ containing eff $(\mathcal{E})$.

Proof. We first show that $\bmod _{\text {adm }}(\mathcal{E})$ is a resolving subcategory of $\mathcal{P}^{2}(\mathcal{E})$. Note that $\bmod _{\text {adm }}(\mathcal{E})$ is closed under extensions by Proposition 3.4 and is idempotent complete by Corollary 3.15, and therefore closed under direct summands. Also, since $\bmod _{\mathrm{adm}}(\mathcal{E})$ contains the representable functors, it is generating in $\mathcal{P}^{2}(\mathcal{E})$. To show closure under kernels of deflations, it suffices by [33, Lemma 2.5] to show that if $0 \rightarrow K \rightarrow \operatorname{Hom}_{\mathcal{E}}(-, E) \stackrel{p}{\rightarrow} G \rightarrow 0$ is an exact sequence with $G \in \bmod _{\mathrm{adm}}(\mathcal{E})$, then $K \in \bmod _{\mathrm{adm}}(\mathcal{E})$. To this end, let $0 \rightarrow F \rightarrow G \stackrel{q}{\rightarrow} H \rightarrow 0$ be a conflation with $F \in \operatorname{eff}(\mathcal{E})$ and $H \in \mathcal{F}$. Then we get a commutative diagram

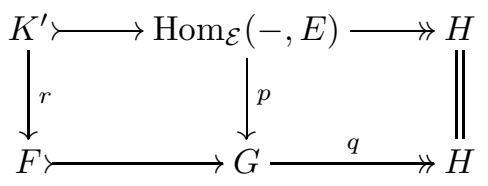

where the rows are conflations. It follows that the left hand square is a pullback square, so $r$ is an epimorphism and $\operatorname{ker}(r) \cong \operatorname{ker}(p) \cong K$. Since $H$ has projective dimension $\leq 1$, it follows that $K^{\prime}$ is projective, and hence $K^{\prime} \cong \operatorname{Hom}_{\mathcal{E}}\left(-, E^{\prime}\right)$ for some $E^{\prime} \in \mathcal{E}$ since $\mathcal{E}$ is idempotent complete. Hence $K^{\prime} \in \bmod _{\mathrm{adm}}(\mathcal{E})$, and therefore $r$ is admissible in $\bmod _{\mathrm{adm}}(\mathcal{E})$ by Proposition 3.5. Hence $K \cong \operatorname{ker}(r) \in$ $\bmod _{\mathrm{adm}}(\mathcal{E})$, so $\bmod _{\mathrm{adm}}(\mathcal{E})$ is closed under kernels of deflations and therefore a resolving subcategory of $\mathcal{P}^{2}(\mathcal{E})$.

Now let $\mathcal{X} \in \mathcal{P}^{2}(\mathcal{E})$ be a resolving subcategory containing eff $(\mathcal{E})$. Then by Proposition 4.4.(3) we have that $\mathcal{X}$ must contain $\mathcal{F}$. Since $(\operatorname{eff}(\mathcal{E}), \mathcal{F})$ is a torsion pair in $\bmod _{\text {adm }}(\mathcal{E})$ and $\mathcal{X}$ is closed under extensions, $\mathcal{X}$ must contain $\bmod _{\mathrm{adm}}(\mathcal{E})$. This proves the claim.

\subsection{The Auslander-Bridger transpose}

Fix an idempotent complete additive category $\mathcal{C}$. We let $\underline{\bmod }(\mathcal{C})$ denote the projectively stable category of $\bmod (\mathcal{C})$. Explicitly, $\underline{\bmod }(\mathcal{C})$ has the same objects as $\bmod (\mathcal{C})$, and

$$
\operatorname{Hom}_{\underline{\bmod }(\mathcal{C})}(F, G)=\operatorname{Hom}_{\bmod (\mathcal{C})}(F, G) / \mathcal{P}(F, G)
$$


where $\mathcal{P}(F, G)$ denotes the set of morphisms $F \rightarrow G$ factoring through a representable functor.

We now recall the Auslander-Bridger transpose, which was first introduced and studied in [6] and [10].

Definition 5.5. The Auslander-Bridger transpose is a functor

$$
\operatorname{Tr}: \underline{\bmod }(\mathcal{C}) \rightarrow \underline{\bmod }\left(\mathcal{C}^{\text {op }}\right)^{\text {op }}
$$

defined as follows:

1. For each object $F \in \underline{\bmod }(\mathcal{C})$ choose a projective presentation

$$
\operatorname{Hom}_{\mathcal{C}}(-, X) \stackrel{\operatorname{Hom}_{\mathcal{C}}(-, f)}{\longrightarrow} \operatorname{Hom}_{\mathcal{C}}(-, Y) \rightarrow F \rightarrow 0
$$

in $\bmod (\mathcal{C})$ and set

$$
\operatorname{Tr}(F)=\operatorname{coker}\left(\operatorname{Hom}_{\mathcal{C}}(Y,-) \stackrel{\operatorname{Hom}_{\mathcal{C}}(f,-)}{\longrightarrow} \operatorname{Hom}_{\mathcal{C}}(X,-)\right) .
$$

2. For each morphism morphism $\underline{\phi}: F \rightarrow G$ in $\underline{\bmod }(\mathcal{C})$ choose a representative $\phi: F \rightarrow G$ in $\bmod (\mathcal{C})$ and a lift

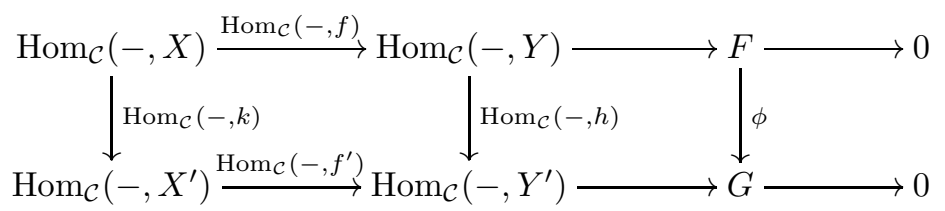

and define $\operatorname{Tr}(\phi): \operatorname{Tr}(F) \rightarrow \operatorname{Tr}(G)$ to be the image in $\underline{\bmod }(\mathcal{C})$ of the unique morphism in $\bmod (\mathcal{C})$ making the diagram

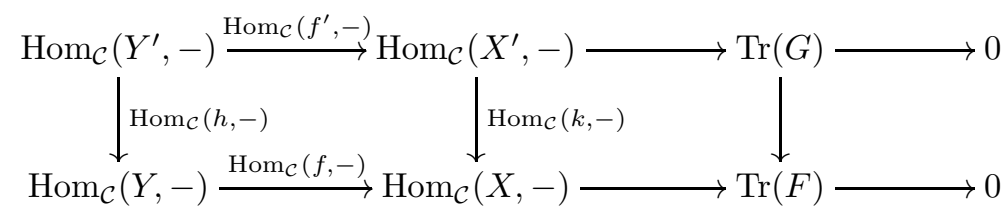

commutative.

By definition we have that $\operatorname{Tr}^{2} \cong 1_{\underline{\bmod }(\mathcal{C})}$, and $\operatorname{Tr}$ therefore induces an equivalence between $\underline{\bmod }(\mathcal{C})$ and $\bmod \left(\mathcal{C}^{\mathrm{op}}\right)^{\mathrm{op}}$. Since $\operatorname{Tr}$ is a functor to the projectively stable category, the object $\operatorname{Tr}(F)$ is well-defined in $\bmod (\mathcal{C})$ up to projective summands. By abuse of notation, we sometimes write $\operatorname{Tr}(F)$ for a choice of an object in $\bmod \left(\mathcal{C}^{\mathrm{op}}\right)$ which is isomorphic in $\underline{\bmod }\left(\mathcal{C}^{\mathrm{op}}\right)$ to the image of $\operatorname{Tr}: \underline{\bmod }(\mathcal{C}) \rightarrow \underline{\bmod }\left(\mathcal{C}^{\mathrm{op}}\right)^{\text {op }}$ at $F \in \underline{\bmod }(\mathcal{C})$.

In the following, for a functor $F \in \operatorname{Mod}(\mathcal{C})$ we let $F^{*} \in \operatorname{Mod}\left(\mathcal{C}^{\text {op }}\right)$ denote the functor given by

$$
F^{*}(X)=\operatorname{Hom}_{\operatorname{Mod}(\mathcal{C})}\left(F, \operatorname{Hom}_{\mathcal{C}}(-, X)\right)
$$

and we let $\mathrm{ev}_{F}: F \rightarrow F^{* *}$ denote the natural transformation sending $x \in F(X)$ to

$$
\operatorname{ev}_{F}(x): F^{*} \rightarrow \operatorname{Hom}_{\mathcal{C}}(X,-) \quad \operatorname{ev}_{F}(x)(\psi)=\psi(x) .
$$

Finally, for $i>0$ we let $\operatorname{Ext}_{\mathcal{C}}^{i}(F, \mathcal{C}) \in \operatorname{Mod}\left(\mathcal{C}^{\text {op }}\right)$ denote the functor given by

$$
\operatorname{Ext}_{\mathcal{C}}^{i}(F, \mathcal{C})(X)=\operatorname{Ext}_{\operatorname{Mod}(\mathcal{C})}^{i}\left(F, \operatorname{Hom}_{\mathcal{C}}(-, X)\right) .
$$

Proposition 5.6 (Proposition 6.3 in $[6]$ ). For any $F \in \bmod (\mathcal{C})$ we have an exact sequence

$$
0 \rightarrow \operatorname{Ext}_{\mathcal{C}^{\mathrm{op}}}^{1}\left(\operatorname{Tr}(F), \mathcal{C}^{\mathrm{op}}\right) \rightarrow F \stackrel{\mathrm{ev}_{F}}{\longrightarrow} F^{* *} \rightarrow \operatorname{Ext}_{\mathcal{C}^{\mathrm{op}}}^{2}\left(\operatorname{Tr}(F), \mathcal{C}^{\mathrm{op}}\right) \rightarrow 0 .
$$

We end this subsection by recalling the definition of the grade, which was first introduced in [57].

Definition 5.7. The grade of $F \in \operatorname{Mod}(\mathcal{C})$, denoted grade $F$, is the biggest integer $i \geq 0$ such that $\operatorname{Ext}_{\operatorname{Mod}(\mathcal{C})}^{j}\left(F, \operatorname{Hom}_{\mathcal{C}}(-, X)\right)=0$ for all $j<i$ and $X \in \mathcal{C}$. Here we let $\operatorname{Ext}_{\operatorname{Mod}(\mathcal{C})}^{0}\left(F, \operatorname{Hom}_{\mathcal{C}}(-, X)\right):=$ $\left.\operatorname{Hom}_{\operatorname{Mod}(\mathcal{C})}\left(F, \operatorname{Hom}_{\mathcal{C}}(-, X)\right)\right)$. 


\subsection{Main theorem}

Fix an idempotent complete additive category $\mathcal{C}$. We are now ready to state our main theorem relating subcategories of $\bmod (\mathcal{C})$ with exact structures on $\mathcal{C}$. Here, for a subcategory $\mathcal{X}$ of $\bmod (\mathcal{C})$ we $\operatorname{let} \operatorname{Tr}(\mathcal{X})$ denote the subcategory of $\bmod \left(\mathcal{C}^{\text {op }}\right)$ consisting of all objects $G$ which are isomorphism in $\underline{\bmod }\left(\mathcal{C}^{\text {op }}\right)$ to an object $\operatorname{Tr}(F)$ with $F \in \mathcal{X}$. Since we encounter multiple exact structures on $\mathcal{C}$, we use the letters $\mathbb{C}, \mathbb{D}$ and $\mathbb{E}$ for classes of conflations in $\mathcal{C}$. Thus a conflation category or an exact category is written as a pair $(\mathcal{C}, \mathbb{C})$, and the associated Auslander exact category is denoted by $\bmod _{\text {adm }}(\mathbb{C})$.

Theorem 5.8. Let $\mathcal{C}$ be an idempotent complete additive category. The association $\mathbb{C} \mapsto \bmod _{\mathrm{adm}}(\mathbb{C})$ gives a bijection between:

1. Classes of conflations $\mathbb{C}$ in $\mathcal{C}$ such that $(\mathcal{C}, \mathbb{C})$ is an exact category;

2. Subcategories $\mathcal{X}$ of $\bmod (\mathcal{C})$ satisfying the following:

(a) $\mathcal{X}$ is a resolving subcategory of $\mathcal{P}^{2}(\mathcal{C})$ and $\operatorname{Tr}(\mathcal{X})$ is a resolving subcategory of $\mathcal{P}^{2}\left(\mathcal{C}^{\mathrm{op}}\right)$;

(b) $\mathcal{X}$ and $\operatorname{Tr}(\mathcal{X})$ have no objects of grade 1 .

Our goal in this section is to prove Theorem 5.8 .

Lemma 5.9. Assume $\mathcal{X}$ is a subcategory of $\bmod (\mathcal{C})$ satisfying condition $(2 \mathrm{a})$ in Theorem 5.8 , and let $F \in \mathcal{X}$. The following hold:

1. $F^{*} \cong \operatorname{Hom}_{\mathcal{C}}(Z,-)$ for some $Z \in \mathcal{C}$;

2. The morphism $\mathrm{ev}_{F}: F \rightarrow F^{* *}$ is admissible in $\mathcal{X}$;

3. $\operatorname{Ext}_{\mathcal{C}}^{1}(F, \mathcal{C}) \in \operatorname{Tr}(\mathcal{X})$ and $\operatorname{Ext}_{\mathcal{C}}^{2}(F, \mathcal{C}) \in \operatorname{Tr}(\mathcal{X})$.

Proof. 1. Recall that there exists an exact sequence

$$
0 \rightarrow F^{*} \rightarrow \operatorname{Hom}_{\mathcal{C}}(X,-) \stackrel{\operatorname{Hom}_{\mathcal{C}}(f,-)}{\longrightarrow} \operatorname{Hom}_{\mathcal{C}}(Y,-) \rightarrow \operatorname{Tr}(F) \rightarrow 0 .
$$

Since $\operatorname{Tr}(F) \in \operatorname{Tr}(\mathcal{X}) \in \mathcal{P}^{2}\left(\mathcal{C}^{\text {op }}\right)$, it follows that $F^{*}$ is projective in $\bmod \left(\mathcal{C}^{\text {op }}\right)$ and hence $F^{*} \cong$ $\operatorname{Hom}_{\mathcal{C}}(Z,-)$ for some $Z \in \mathcal{C}$ since $\mathcal{C}$ is idempotent complete.

2. Consider the exact sequence

$$
0 \rightarrow \operatorname{Ext}_{\mathcal{C}^{\mathrm{op}}}^{1}\left(\operatorname{Tr}(F), \mathcal{C}^{\mathrm{op}}\right) \rightarrow F \stackrel{\mathrm{ev}_{F}}{\longrightarrow} F^{* *} \rightarrow \operatorname{Ext}_{\mathcal{C}^{\mathrm{op}}}^{2}\left(\operatorname{Tr}(F), \mathcal{C}^{\mathrm{op}}\right) \rightarrow 0 .
$$

from Proposition 5.6. Since $F^{* *}$ is representable by part (1), it is contained in $\mathcal{X}$. Therefore, since $\mathcal{X}$ is closed under kernels of epimorphisms, it suffices to show that $\operatorname{Ext}_{\mathcal{C}^{\mathrm{op}}}^{2}\left(\operatorname{Tr}(F), \mathcal{C}^{\mathrm{op}}\right)$ is in $\mathcal{X}$. Now by choosing an exact sequence

$$
0 \rightarrow \operatorname{Hom}_{\mathcal{C}}(Z,-) \rightarrow \operatorname{Hom}_{\mathcal{C}}(Y,-) \stackrel{\operatorname{Hom}_{\mathcal{C}}(f,-)}{\longrightarrow} \operatorname{Hom}_{\mathcal{C}}(X,-) \rightarrow \operatorname{Tr}(F) \rightarrow 0
$$

we immediately see that $\operatorname{Ext}_{\mathcal{C}^{\text {op }}}^{2}\left(\operatorname{Tr}(F), \mathcal{C}^{\text {op }}\right) \cong \operatorname{Tr}\left(\operatorname{im}\left(\operatorname{Hom}_{\mathcal{C}}(f,-)\right)\right)$. Since $\operatorname{Tr}(\mathcal{X})$ is closed under kernels of epimorphisms, we get that $\operatorname{im}\left(\operatorname{Hom}_{\mathcal{C}}(f,-)\right) \in \operatorname{Tr}(\mathcal{X})$, and hence $\operatorname{Ext}_{\mathcal{C}^{\mathrm{op}}}^{2}\left(\operatorname{Tr}(F), \mathcal{C}^{\mathrm{op}}\right) \in \mathcal{X}$.

3. Proposition 5.6 applied to $\operatorname{Tr}(F)$ gives an exact sequence

$$
0 \rightarrow \operatorname{Ext}_{\mathcal{C}}^{1}(F, \mathcal{C}) \rightarrow \operatorname{Tr}(F) \stackrel{\mathrm{ev}_{\operatorname{Tr}(F)}}{\longrightarrow} \operatorname{Tr}(F)^{* *} \rightarrow \operatorname{Ext}_{\mathcal{C}}^{2}(F, \mathcal{C}) \rightarrow 0
$$

Hence, the claim follows from part (2) applied to $\operatorname{Tr}(F)$ and $\operatorname{Tr}(\mathcal{X})$.

Lemma 5.10. Assume $\mathcal{X}$ is a subcategory of $\bmod (\mathcal{C})$ satisfying condition (2a) in Theorem 5.8. The following are equivalent:

1. For all $F \in \mathcal{X}$ of projective dimension $\leq 1$, the $\operatorname{map} \mathrm{ev}_{F}: F \rightarrow F^{* *}$ is an inflation in $\mathcal{X}$;

2. $\operatorname{Tr}(\mathcal{X})$ has no objects of grade 1 . 
Proof. For each $F \in \mathcal{X}$ choose a projective presentation $\operatorname{Hom}_{\mathcal{C}}(-, Y) \stackrel{\operatorname{Hom}_{\mathcal{C}}(-, f)}{\longrightarrow} \operatorname{Hom}_{\mathcal{C}}(-, X) \rightarrow F \rightarrow 0$ such that $\operatorname{Hom}_{\mathcal{C}}(-, f)$ is a monomorphism if $F$ has projective dimension $\leq 1$. Define $\operatorname{Tr}(F)$ via the exact sequence $\operatorname{Hom}_{\mathcal{C}}(X,-) \stackrel{\operatorname{Hom}_{\mathcal{C}}(f,-)}{\longrightarrow} \operatorname{Hom}_{\mathcal{C}}(Y,-) \rightarrow \operatorname{Tr}(F)$. Then clearly $F$ has projective dimension $\leq 1$ if and only if grade $\operatorname{Tr}(F)>0$. Furthermore, ev ${ }_{F}$ is an inflation in $\mathcal{X}$ if and only if $\operatorname{Ext}_{\mathcal{C}^{\text {op }}}^{1}\left(\operatorname{Tr}(F), \mathcal{C}^{\text {op }}\right) \cong 0$ by Proposition 5.6 and Lemma 5.9.(2). Hence, $\mathrm{ev}_{F}$ is an inflation for any object $F \in \mathcal{X}$ of projective dimension $\leq 1$ if and only if no object of the form $\operatorname{Tr}(F)$ with $F \in \mathcal{X}$ has grade 1 . Since any object in $\operatorname{Tr}(\mathcal{X})$ is isomorphic in $\underline{\bmod }\left(\mathcal{C}^{\text {op }}\right)$ to an object of the form $\operatorname{Tr}(F)$ with $F \in \mathcal{X}$, this is equivalent to $\operatorname{Tr}(\mathcal{X})$ having no objects of grade 1 .

Definition 5.11. Assume $\mathcal{X}$ is a subcategory of $\bmod (\mathcal{C})$ satisfying condition $(2 \mathrm{a})$ and $(2 \mathrm{~b})$ in Theorem 5.8 , and let $f: X \rightarrow Y$ be a morphism in $\mathcal{C}$.

1. $f$ is an $\mathcal{X}$-inflation if $\operatorname{Hom}_{\mathcal{C}}(-, f): \operatorname{Hom}_{\mathcal{C}}(-, X) \rightarrow \operatorname{Hom}_{\mathcal{C}}(-, Y)$ is an inflation in $\mathcal{X}$;

2. $f$ is an $\mathcal{X}$-deflation if $\operatorname{Hom}_{\mathcal{C}}(f,-): \operatorname{Hom}_{\mathcal{C}}(Y,-) \rightarrow \operatorname{Hom}_{\mathcal{C}}(X,-)$ is an inflation in $\operatorname{Tr}(\mathcal{X})$;

3. $f$ is $\mathcal{X}$-admissible if $\operatorname{Hom}_{\mathcal{C}}(-, f): \operatorname{Hom}_{\mathcal{C}}(-, X) \rightarrow \operatorname{Hom}_{\mathcal{C}}(-, Y)$ is admissible in $\mathcal{X}$.

Proposition 5.12. Assume $\mathcal{X}$ is a subcategory of $\bmod (\mathcal{C})$ satisfying condition $(2 \mathrm{a})$ and $(2 \mathrm{~b})$ in Theorem 5.8. Then there exists a unique conflation category $(\mathcal{C}, \mathbb{C})$ with inflations and deflations the $\mathcal{X}$ inflations and $\mathcal{X}$-deflations, respectively.

Proof. Let $f$ be an $\mathcal{X}$-inflation, and consider the exact sequences

$$
\begin{array}{r}
0 \rightarrow \operatorname{Hom}_{\mathcal{C}}(-, X) \stackrel{\operatorname{Hom}_{\mathcal{C}}(-, f)}{\longrightarrow} \operatorname{Hom}_{\mathcal{C}}(-, Y) \rightarrow F \rightarrow 0 \\
0 \rightarrow F^{*} \rightarrow \operatorname{Hom}_{\mathcal{C}}(Y,-) \stackrel{\operatorname{Hom}_{\mathcal{C}}(f,-)}{\longrightarrow} \operatorname{Hom}_{\mathcal{C}}(X,-) \rightarrow \operatorname{Tr}(F) \rightarrow 0 .
\end{array}
$$

Since $F^{*} \cong \operatorname{Hom}_{\mathcal{C}}(Z,-)$ by Lemma 5.9.(1), we get that $f$ admits a cokernel $f: Y \rightarrow Z$ in $\mathcal{C}$ which is an $\mathcal{X}$-deflation. Now since $F$ has projective dimension $\leq 1$, the $\operatorname{map} \operatorname{ev}_{F}: F \rightarrow F^{* *} \cong \operatorname{Hom}_{\mathcal{C}}(Z,-)$ is an inflation in $\mathcal{X}$ by Lemma 5.10. Hence the sequence $0 \rightarrow \operatorname{Hom}_{\mathcal{C}}(-, X) \rightarrow \operatorname{Hom}_{\mathcal{C}}(-, Y) \rightarrow \operatorname{Hom}_{\mathcal{C}}(-, Z)$ is exact, so $f$ is the kernel of $g$. This together with the dual argument shows that the $\mathcal{X}$-inflations and $\mathcal{X}$-deflations makes $\mathcal{C}$ into a conflation category.

Proposition 5.13. Assume $\mathcal{X}$ is a subcategory of $\bmod (\mathcal{C})$ satisfying condition $(2 \mathrm{a})$ and $(2 \mathrm{~b})$ in Theorem 5.8. Then the conflation category in Proposition 5.12 is an exact category.

Proof. Since the $\mathcal{X}$-inflations and $\mathcal{X}$-deflations clearly satisfy axioms $(\mathbf{L 0}),(\mathbf{L} 1)$ and $(\mathbf{R} 0),(\mathbf{R} 1)$ for an exact category, it only remains to show that axioms $(\mathbf{L 2})$ and $(\mathbf{R 2})$ hold. We only prove $(\mathbf{L} 2)$ since $(\mathbf{R 2})$ is dual. To this end, let $f: X \rightarrow Y$ be an $\mathcal{X}$-inflation and let $g: X \rightarrow Z$ be a morphism in $\mathcal{C}$. Taking the pushout of $\operatorname{Hom}_{\mathcal{C}}(-, f): \operatorname{Hom}_{\mathcal{C}}(-, X) \rightarrow \operatorname{Hom}_{\mathcal{C}}(-, Y)$ along $\operatorname{Hom}_{\mathcal{C}}(-, g): \operatorname{Hom}_{\mathcal{C}}(-, X) \rightarrow \operatorname{Hom}_{\mathcal{C}}(-, Z)$, we get a commutative diagram

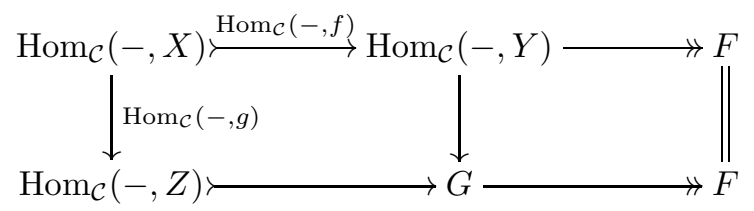

where the rows are exact sequences. Since the left hand square is a pullback and a pushout square, we have an exact sequence

$$
0 \rightarrow \operatorname{Hom}_{\mathcal{C}}(-, X) \stackrel{\left(\begin{array}{l}
\operatorname{Hom}_{\mathcal{C}}(-, f) \\
\operatorname{Hom}_{\mathcal{C}}(-, g)
\end{array}\right)}{\longrightarrow} \operatorname{Hom}_{\mathcal{C}}(-, Y) \oplus \operatorname{Hom}_{\mathcal{C}}(-, Z) \rightarrow G \rightarrow 0
$$

and hence $G$ has projective dimension $\leq 1$. Therefore by Lemma 5.10 the map $\operatorname{ev}_{G}: G \rightarrow G^{* *}$ is an inflation in $\mathcal{X}$. Furthermore, $G^{*} \cong \operatorname{Hom}_{\mathcal{C}}(W,-)$ for some object $W \in \mathcal{C}$ by Lemma 5.9.(1). Let $h: Z \rightarrow W$ and $k: Y \rightarrow W$ be the unique morphisms in $\mathcal{C}$ so that $\operatorname{Hom}_{\mathcal{C}}(-, h)$ and $\operatorname{Hom}_{\mathcal{C}}(-, k)$ are equal to the composites $\operatorname{Hom}_{\mathcal{C}}(-, Z) \longmapsto G \stackrel{\mathrm{ev}_{G}}{\longrightarrow} G^{* *} \cong \operatorname{Hom}_{\mathcal{C}}(-, W)$ and $\operatorname{Hom}_{\mathcal{C}}(-, Y) \rightarrow G \stackrel{\mathrm{ev}_{G}}{\longrightarrow} G^{* *} \cong \operatorname{Hom}_{\mathcal{C}}(-, W)$, 
respectively. Since $\operatorname{Hom}_{\mathcal{C}}(-, h)$ is a composite of inflations in $\mathcal{X}$, it follows that $h$ is an $\mathcal{X}$-inflation. Now consider the commutative square



It only remains to show that this is a pushout square. But this is true since applying $(-)^{*}$ to $(3)$ gives the left exact sequence

$$
0 \rightarrow \operatorname{Hom}_{\mathcal{C}}(W,-) \stackrel{\left(\begin{array}{l}
\operatorname{Hom}_{\mathcal{C}}(k,-) \\
\operatorname{Hom}_{\mathcal{C}}(h,-)
\end{array}\right)}{\longrightarrow} \operatorname{Hom}_{\mathcal{C}}(Y,-) \oplus \operatorname{Hom}_{\mathcal{C}}(Z,-) \stackrel{\left(\operatorname{Hom}_{\mathcal{C}}(f,-) \operatorname{Hom}_{\mathcal{C}}(g,-)\right)}{\longrightarrow} \operatorname{Hom}_{\mathcal{C}}(X,-)
$$

Proposition 5.14. Assume $\mathcal{X}$ is a subcategory of $\bmod (\mathcal{C})$ satisfying condition $(2 \mathrm{a})$ and $(2 \mathrm{~b})$ in Theorem 5.8, and let $(\mathcal{C}, \mathbb{C})$ be the exact category given by the $\mathcal{X}$-inflations and $\mathcal{X}$-deflations. The following hold:

1. The $\mathcal{X}$-admissible morphisms in $\mathcal{C}$ are precisely the admissible morphisms in $(\mathcal{C}, \mathbb{C})$;

2. $\bmod _{\mathrm{adm}}(\mathbb{C})=\mathcal{X}$.

Proof. 1. Let $f: X \rightarrow Y$ be a morphism in $\mathcal{C}$. If $f$ is admissible in $(\mathcal{C}, \mathbb{C})$, then it can be written as a composite $f=f_{2} \circ f_{1}$ where $f_{1}$ is an $\mathcal{X}$-deflation and $f_{2}$ is an $\mathcal{X}$-inflation. By definition $\operatorname{Hom}_{\mathcal{C}}\left(f_{1},-\right)$ is an inflation in $\operatorname{Tr}(\mathcal{X})$, and hence $\operatorname{Hom}_{\mathcal{C}}\left(-, f_{1}\right)$ must be admissible in $\mathcal{X}$. Since $\operatorname{Hom}_{\mathcal{C}}\left(-, f_{2}\right)$ is an inflation in $\mathcal{X}$, the composite

$$
\operatorname{Hom}_{\mathcal{C}}(-, f)=\operatorname{Hom}_{\mathcal{C}}\left(-, f_{2}\right) \circ \operatorname{Hom}_{\mathcal{C}}\left(-, f_{1}\right)
$$

is admissible in $\mathcal{X}$. Hence $f$ is $\mathcal{X}$-admissible.

Now assume $f$ is $\mathcal{X}$-admissible. First note that $f$ has a kernel which is an $\mathcal{X}$-inflation and a cokernel which is an $\mathcal{X}$-deflation, since $\operatorname{Hom}_{\mathcal{C}}(-, f)$ and $\operatorname{Hom}_{\mathcal{C}}(-, f)$ are admissible in $\mathcal{X}$ with kernels being representable functors. Therefore $f$ has a coimage coim $(f)$ obtained by taking the cokernel of the inclusion $\operatorname{ker}(f) \longmapsto X$, and an image $\operatorname{im}(f)$ obtained by taking the kernel of the projection $Y \rightarrow \operatorname{coker}(f)$. Hence we get a canonical map coim $(f) \rightarrow \operatorname{im}(f)$, and it suffices to show that this is an isomorphism. To this end, let $g: X \rightarrow \operatorname{coim}(f)$ denote the given $\mathcal{X}$-deflation, and let $h: \operatorname{coim}(f) \rightarrow Y$ denote the composite $\operatorname{coim}(f) \rightarrow \operatorname{im}(f) \rightarrow Y$. Consider the commutative diagram with exact rows

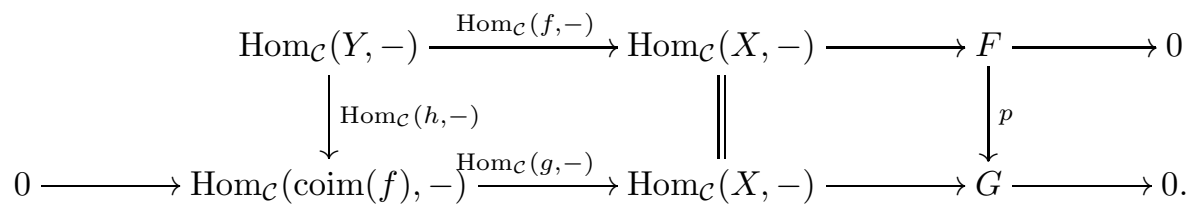

Since $f$ and $g$ are $\mathcal{X}$-admissible, $F$ and $G$ are in $\operatorname{Tr}(\mathcal{X})$. By the snake lemma $p$ is an epimorphism and $\operatorname{coker}\left(\operatorname{Hom}_{\mathcal{C}}(h,-)\right) \cong \operatorname{ker}(p)$. Since $\operatorname{Tr}(\mathcal{X})$ is closed under kernels of epimorphisms, $\operatorname{ker}(p)$ is contained in $\operatorname{Tr}(\mathcal{X})$, and hence $h$ is $\mathcal{X}$-admissible. Let $i: \operatorname{ker}(h) \rightarrow \operatorname{coim}(f)$ denote the inclusion of the kernel, and let $j: E \rightarrow X$ denote the pullback of $i$ along $g$. Then we get a commutative diagram

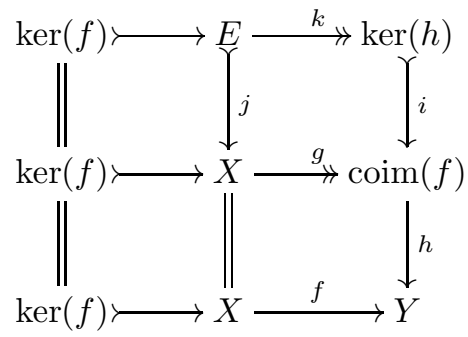


where the two upper rows are conflations in $(\mathcal{C}, \mathbb{C})$. Hence $f \circ j=0$, so $j$ factors through $\operatorname{ker}(f)$. It follows that $i$ must be 0 , so $\operatorname{ker}(h) \cong 0$ since $i$ is an inflation in $(\mathcal{C}, \mathbb{C})$. Hence $h$ is an $\mathcal{X}$-inflation. Since $h$ can be written as a composite $\operatorname{coim}(f) \rightarrow \operatorname{im}(f) \rightarrow Y$ and $\mathcal{C}$ is weakly idempotent complete, the map $\operatorname{coim}(f) \rightarrow \operatorname{im}(f)$ must be an $\mathcal{X}$-inflation by [24, Proposition 7.6]. Dually, one shows that the map $\operatorname{coim}(f) \rightarrow \operatorname{im}(f)$ is an $\mathcal{X}$-deflation. Since a map in an exact category which is both an inflation and a deflation must be an isomorphism, the claim follows.

2. This follows immediately from part (1).

Proof of Theorem 5.8. If $(\mathcal{C}, \mathbb{C})$ is an exact category, then $\bmod _{\mathrm{adm}}(\mathbb{C})$ and $\operatorname{Tr}\left(\bmod _{\mathrm{adm}}(\mathbb{C})\right)=\bmod _{\mathrm{adm}}(\mathbb{C}$ op $)$ are resolving by Proposition 5.4. Also any $F \in \bmod _{\text {adm }}(\mathbb{C})$ and $G \in \bmod _{\text {adm }}(\mathbb{C}$ op $)$ of grade $>0$ must satisfy

$$
\operatorname{Ext}_{\operatorname{Mod}(\mathcal{C})}^{1}\left(F, \operatorname{Hom}_{\mathcal{C}}(-, X)\right)=0 \quad \text { and } \quad \operatorname{Ext}_{\operatorname{Mod}\left(\mathcal{C}^{\mathrm{op}}\right)}^{1}\left(F, \operatorname{Hom}_{\mathcal{C}}(X,-)\right)=0
$$

for all $X \in \mathcal{C}$ by Proposition 3.14.(3). Therefore $F$ and $G$ must have grade $>1$. This shows that $\bmod _{\mathrm{adm}}(\mathbb{C})$ satisfies $(2 \mathrm{a})$ and $(2 \mathrm{~b})$ in Theorem 5.8. The fact that the association $\mathbb{C} \mapsto \bmod _{\mathrm{adm}}(\mathbb{C})$ is a bijection follows from Proposition 5.12, Proposition 5.13 and Proposition 5.14.

We end this section by considering exact structures on an abelian category $\mathcal{A}$.

Corollary 5.15. Let $\mathcal{A}$ be an abelian category. Then there exists a bijection between the following:

1. Exact structures on $\mathcal{A}$;

2. Resolving subcategory $\mathcal{X}$ of $\bmod (\mathcal{A})$ for which $\operatorname{Tr}(\mathcal{X})$ is a resolving subcategory of $\bmod \left(\mathcal{A}^{\mathrm{op}}\right)$.

Proof. Since $\mathcal{A}$ is abelian, $\bmod (\mathcal{A})=\mathcal{P}^{2}(\mathcal{A})$ and $\bmod \left(\mathcal{A}^{\mathrm{op}}\right)=\mathcal{P}^{2}\left(\mathcal{A}^{\mathrm{op}}\right)$, and $\bmod (\mathcal{A})$ and $\bmod \left(\mathcal{A}^{\mathrm{op}}\right)$ have no objects of grade 1 . Hence, the claim follows from Theorem 5.8.

\section{References}

[1] M. Artin and J.-L. Verdier, Reflexive modules over rational double points, Math. Ann. 270 (1985), no. $1,79-82$.

[2] M. Auslander and $\varnothing$. Solberg, Gorenstein algebras and algebras with dominant dimension at least 2, Comm. Algebra 21 (1993), no. 11, 3897-3934.

[3] - Relative homology and representation theory. I. Relative homology and homologically finite subcategories, Comm. Algebra 21 (1993), no. 9, 2995-3031.

[4] _ Relative homology and representation theory. II. Relative cotilting theory, Comm. Algebra 21 (1993), no. 9, 3033-3079.

[5] _ Relative homology and representation theory. III. Cotilting modules and Wedderburn correspondence, Comm. Algebra 21 (1993), no. 9, 3081-3097.

[6] Maurice Auslander, Coherent functors, Proc. Conf. Categorical Algebra (La Jolla, Calif., 1965), Springer, New York, 1966, pp. 189-231.

[7] , Functors and morphisms determined by objects, Representation theory of algebras (Proc. Conf., Temple Univ., Philadelphia, Pa., 1976), 1978, pp. 1-244. Lecture Notes in Pure Appl. Math., Vol. 37.

[8] _ Isolated singularities and existence of almost split sequences, Representation theory, II (Ottawa, Ont., 1984), Lecture Notes in Math., vol. 1178, Springer, Berlin, 1986, pp. 194-242.

[9] _ Rational singularities and almost split sequences, Trans. Amer. Math. Soc. 293 (1986), no. 2, 511-531.

[10] Maurice Auslander and Mark Bridger, Stable module theory, Memoirs of the American Mathematical Society, No. 94, American Mathematical Society, Providence, R.I., 1969. 
[11] Maurice Auslander and Idun Reiten, Stable equivalence of dualizing R-varieties, Advances in Math. 12 (1974), 306-366.

[12] - Representation theory of Artin algebras. VI. A functorial approach to almost split sequences, Comm. Algebra 6 (1978), no. 3, 257-300.

[13] _ Almost split sequences for $\mathbf{Z}$-graded rings, Singularities, representation of algebras, and vector bundles (Lambrecht, 1985), Lecture Notes in Math., vol. 1273, Springer, Berlin, 1987, pp. 232243.

[14] Cohen-Macaulay modules for graded Cohen-Macaulay rings and their completions, Commutative algebra (Berkeley, CA, 1987), Math. Sci. Res. Inst. Publ., vol. 15, Springer, New York, 1989, pp. 21-31.

[15] _ _ Applications of contravariantly finite subcategories, Adv. Math. 86 (1991), no. 1, 111-152.

[16] _ Cohen-Macaulay and Gorenstein Artin algebras, Representation theory of finite groups and finite-dimensional algebras (Bielefeld, 1991), Progr. Math., vol. 95, Birkhäuser, Basel, 1991, pp. 221245 .

[17] Rose-Line Baillargeon, Thomas Brüstle, Mikhail Gorsky, and Souheila Hassoun, On the lattice of weakly exact structures, arXiv:2009.10024 (2020).

[18] Apostolos Beligiannis, On the Freyd categories of an additive category, Homology Homotopy Appl. 2 (2000), 147-185.

[19] _ On algebras of finite Cohen-Macaulay type, Adv. Math. 226 (2011), no. 2, 1973-2019.

[20] Thomas Brüstle, Souheila Hassoun, and Aran Tattar, Intersections, sums, and the Jordan-Hölder property for exact categories, arXiv:2006.03505 (2020).

[21] Thomas Brüstle, Souheila Hassoun, Denis Langford, and Sunny Roy, Reduction of exact structures, J. Pure Appl. Algebra 224 (2020), no. 4, 106212, 29.

[22] Aslak Bakke Buan, Closed subbifunctors of the extension functor, J. Algebra 244 (2001), no. 2, 407-428.

[23] R.-O. Buchweitz, G.-M. Greuel, and F.-O. Schreyer, Cohen-Macaulay modules on hypersurface singularities. II, Invent. Math. 88 (1987), no. 1, 165-182.

[24] Theo Bühler, Exact categories, Expo. Math. 28 (2010), no. 1, 1-69.

[25] M. C. R. Butler and G. Horrocks, Classes of extensions and resolutions, Philos. Trans. Roy. Soc. London Ser. A 254 (1961/62), 155-222.

[26] Manuel Cardenas, Localization for exact categories, dissertation, Binghampton University, 1998.

[27] Jon F. Carlson, Book Review: Methods of representation theory with applications to finite groups and orders, vol. 1, Bull. Amer. Math. Soc. (N.S.) 8 (1983), no. 1, 112-116.

[28] Xiao-Wu Chen, An Auslander-type result for Gorenstein-projective modules, Adv. Math. 218 (2008), no. 6, 2043-2050.

[29] Xiao-Wu Chen, Dawei Shen, and Guodong Zhou, The Gorenstein-projective modules over a monomial algebra, Proc. Roy. Soc. Edinburgh Sect. A 148 (2018), no. 6, 1115-1134.

[30] Peter Dräxler, Idun Reiten, Sverre O. Smalø, and Ø yvind Solberg, Exact categories and vector space categories, Trans. Amer. Math. Soc. 351 (1999), no. 2, 647-682, With an appendix by B. Keller.

[31] Samuel Eilenberg and J. C. Moore, Foundations of relative homological algebra, Mem. Amer. Math. Soc. 55 (1965), 39. 
[32] Edgar E. Enochs and Overtoun M. G. Jenda, Relative homological algebra, De Gruyter Expositions in Mathematics, vol. 30, Walter de Gruyter \& Co., Berlin, 2000.

[33] Haruhisa Enomoto, Classifying exact categories via Wakamatsu tilting, J. Algebra 485 (2017), 1-44.

[34] _ Classifications of exact structures and Cohen-Macaulay-finite algebras, Adv. Math. 335 (2018), 838-877.

[35] Haruhisa Enomoto, Classifying substructures of extriangulated categories via Serre subcategories, arXiv:2005.13381 (2020).

[36] Xin Fang and Mikhail Gorsky, Exact structures and degeneration of Hall algebras, arXiv:2005.12130 (2020).

[37] Peter Freyd, Representations in abelian categories, Proc. Conf. Categorical Algebra (La Jolla, Calif., 1965), Springer, New York, 1966, pp. 95-120.

[38] P. Gabriel and M. Zisman, Calculus of fractions and homotopy theory, Ergebnisse der Mathematik und ihrer Grenzgebiete, Band 35, Springer-Verlag New York, Inc., New York, 1967.

[39] Peter Gabriel, Des categories abeliennes, Bull. Soc. Math. France 90 (1962), 323-448.

[40] Alexander Grothendieck, Sur quelques points d'algèbre homologique, Tohoku Math. J. (2) 9 (1957), $119-221$.

[41] Dieter Happel and Luise Unger, Modules of finite projective dimension and cocovers, Math. Ann. 306 (1996), no. 3, 445-457.

[42] Ruben Henrard and Adam-Christiaan van Roosmalen, Derived categories of (one-sided) exact categories and their localizations, arXiv:1903.12647 (2019).

[43] — Localizations of (one-sided) exact categories, arXiv:1903.10861 (2019).

[44] _ On the obscure axiom for one-sided exact categories, arXiv:2010.11293 (2020).

[45] Osamu Iyama, Auslander correspondence, Adv. Math. 210 (2007), no. 1, 51-82.

[46] Osamu Iyama and Michael Wemyss, Maximal modifications and Auslander-Reiten duality for nonisolated singularities, Invent. Math. 197 (2014), no. 3, 521-586.

[47] Dmitry Kaledin and Wendy Lowen, Cohomology of exact categories and (non-)additive sheaves, Adv. Math. 272 (2015), 652-698.

[48] Bernhard Keller, Chain complexes and stable categories, Manuscripta Math. 67 (1990), no. 4, 379417.

[49] Horst Knörrer, Cohen-Macaulay modules on hypersurface singularities. I, Invent. Math. 88 (1987), no. $1,153-164$.

[50] Henning Krause, Deriving Auslander's formula, Doc. Math. 20 (2015), 669-688.

[51] Graham J. Leuschke and Roger Wiegand, Cohen-Macaulay representations, Mathematical Surveys and Monographs, vol. 181, American Mathematical Society, Providence, RI, 2012.

[52] Eduardo N. Marcos, Héctor A. Merklen, and María I. Platzeck, The Grothendieck group of the category of modules of finite projective dimension over certain weakly triangular algebras, Comm. Algebra 28 (2000), no. 3, 1387-1404.

[53] Amnon Neeman, The derived category of an exact category, J. Algebra 135 (1990), no. 2, 388-394.

[54] George Peschke and Tim Van der Linden, The Yoneda isomorphism commutes with homology, J. Pure Appl. Algebra 220 (2016), no. 2, 495-517. 
[55] María Inés Platzeck and Idun Reiten, Modules of finite projective dimension for standardly stratified algebras, Comm. Algebra 29 (2001), no. 3, 973-986.

[56] Daniel Quillen, Higher algebraic K-theory. I, Algebraic $K$-theory, I: Higher $K$-theories (Proc. Conf., Battelle Memorial Inst., Seattle, Wash., 1972), Springer, Berlin, 1973, pp. 85-147. Lecture Notes in Math., Vol. 341.

[57] D. Rees, The grade of an ideal or module, Proc. Cambridge Philos. Soc. 53 (1957), 28-42.

[58] Idun Reiten and Michel Van den Bergh, Two-dimensional tame and maximal orders of finite representation type, Mem. Amer. Math. Soc. 80 (1989), no. 408, viii+72.

[59] Claus Michael Ringel, The Gorenstein projective modules for the Nakayama algebras. I, J. Algebra 385 (2013), 241-261.

[60] Wolfgang Rump, Triads, J. Algebra 280 (2004), no. 2, 435-462.

[61] - On the maximal exact structure on an additive category, Fund. Math. 214 (2011), no. 1, $77-87$.

[62] - Stable short exact sequences and the maximal exact structure of an additive category, Fund. Math. 228 (2015), no. 1, 87-96.

[63] _ The abelian closure of an exact category, J. Pure Appl. Algebra 224 (2020), no. 10, 106395, 29.

[64] Marco Schlichting, Delooping the K-theory of exact categories, Topology 43 (2004), no. 5, 1089-1103.

[65] Yuji Yoshino, Cohen-Macaulay modules over Cohen-Macaulay rings, London Mathematical Society Lecture Note Series, vol. 146, Cambridge University Press, Cambridge, 1990. 